\title{
A Proof of the Standard Completeness for the Involutive Uninorm Logic
}

\author{
SanMin Wang \\ Faculty of Science, Zhejiang Sci-Tech University, Hangzhou 310018, China; \\ wangsanmin@hotmail.com; Tel.: +86-136-5581-2543
}

Received: 20 February 2019; Accepted: 22 March 2019; Published: 27 March 2019

\begin{abstract}
In this paper, we solve a long-standing open problem in the field of fuzzy logics, that is, the standard completeness for the involutive uninorm logic IUL. In fact, we present a uniform method of density elimination for several semilinear substructural logics. Especially, the density elimination for IUL is proved. Then the standard completeness for IUL follows as a lemma by virtue of previous work by Metcalfe and Montagna.
\end{abstract}

Keywords: density elimination; involutive uninorm logic; standard completeness of HpsUL*; semilinear substructural logics; fuzzy logic

MSC: 03B50; 03F05; 03B52; 03B47

\section{Introduction}

The problem of the completeness of Łukasiewicz infinite-valued logic ( $\mathbf{E}$, for short) was posed by Łukasiewicz and Tarski in the 1930s. It was twenty-eight years later that it was syntactically solved by Rose and Rosser [1]. Chang [2] developed at almost the same time a theory of algebraic systems for $\mathbf{E}$, which are called $\mathbf{M V}$-algebras, with an attempt to make $\mathbf{M V}$-algebras correspond to $\mathbf{E}$ as Boolean algebras to the classical two-valued logic. Chang [3] subsequently finished another proof for the completeness of $€$ by virtue of his $\mathbf{M V}$-algebras.

It was Chang who observed that the key role in the structure theory of $\mathbf{M V}$-algebras is not locally finite MV-algebras but linearly ordered ones. The observation was formalized by Hájek [4] who showed the completeness for his basic fuzzy logic (BL for short) with respect to linearly ordered BL-algebras. Starting with the structure of BL-algebras, Hájek [5] reduced the problem of the standard completeness of $\mathbf{B L}$ to two formulas to be provable in BL. Here and thereafter, by the standard completeness we mean that logics are complete with respect to algebras with lattice reduct [0, 1]. Cignoli et al. [6] subsequently proved the standard completeness of BL, i.e., BL is the logic of continuous t-norms and their residua.

Hajek's approach toward fuzzy logic has been extended by Esteva and Godo in [7], where the authors introduced the logic MTL which aims at capturing the tautologies of left-continuous t-norms and their residua. The standard completeness of MTL was proved by Jenei and Montagna in [8], where the major step is to embed linearly ordered MTL-algebras into the dense ones under the situation that the structure of MTL-algebras have been unknown as of yet.

Esteva and Godo's work was further promoted by Metcalfe and Montagna [9] who introduced the uninorm logic UL and involutive uninorm logic (IUL) which aims at capturing tautologies of left-continuous uninorms and their residua and those of involutive left-continuous ones, respectively. Recently, Cintula and Noguera [10] introduced semilinear substructural logics which are substructural logics complete with respect to linearly ordered models. Almost all well-known families of fuzzy logics such as $\mathbf{E}, \mathbf{B L}, \mathbf{M T L}$, UL and IUL belong to the class of semilinear substructural logics. 
Metcalfe and Montagna's method to prove standard completeness for UL and its extensions is of proof theory in nature and consists of two key steps. Firstly, they extended UL with the density rule of Takeuti and Titani [11]:

$$
\frac{\Gamma \vdash(A \rightarrow p) \vee(p \rightarrow B) \vee C}{\Gamma \vdash(A \rightarrow B) \vee C}(D),
$$

where $p$ does not occur in $\Gamma, A, B$ or $C$, and then prove the logics with $(D)$ are complete with respect to algebras with lattice reduct $[0,1]$. Secondly, they give a syntactic elimination of $(D)$ that was formulated as a rule of the corresponding hypersequent calculus.

Hypersequents are a natural generalization of sequents which were introduced independently by Avron [12] and Pottinger [13] and have proved to be particularly suitable for logics with prelinearity $[9,14]$. Following the spirit of Gentzen's cut elimination, Metcalfe and Montagna succeeded to eliminate the density rule for GUL and several extensions of GUL by induction on the height of a derivation of the premise and shifting applications of the rule upwards, but failed for GIUL and therefore left it as an open problem.

There are several relevant works about the standard completeness of IUL as follows. With an attempt to prove the standard completeness of IUL, we generalized Jenei and Montagna's method [15] for IMTL in [16], but our effort was only partially successful. It seems that the subtle reason why it does not work for UL and IUL is the failure of the finite model property of these systems [17]. Jenei [18] constructed several classes of involutive $\mathrm{FL}_{e}$-algebras, as he said, in order to gain a better insight into the algebraic semantic of the substructural logic IUL, and also to the long-standing open problem about its standard completeness. Ciabattoni and Metcalfe [19] introduced the method of density elimination by substitutions which is applicable to a general class of (first-order) hypersequent calculi but fails in the case of GIUL.

We reconsidered Metcalfe and Montagna's proof-theoretic method to investigate the standard completeness of IUL, because they have proved the standard completeness of UL by their method and we cannot prove such a result by the Jenei and Montagna's model-theoretic method. In order to prove the density elimination for GUL, they prove that the following generalized density rule $\left(\mathcal{D}_{1}\right)$ :

$$
\frac{G_{0} \equiv\left\{\Gamma_{i}, \lambda_{i} p \Rightarrow \Delta_{i}\right\}_{i=1 \cdots n}\left|\left\{\Sigma_{k},\left(\mu_{k}+1\right) p \Rightarrow p\right\}_{k=1 \cdots o}\right|\left\{\Pi_{j} \Rightarrow p\right\}_{j=1 \cdots m}}{\mathcal{D}_{1}\left(G_{0}\right) \equiv\left\{\Gamma_{i}, \lambda_{i} \Pi_{j} \Rightarrow \Delta_{i}\right\}_{i=1 \cdots n}^{j=1 \cdots m} \mid\left\{\Sigma_{k}, \mu_{k} \Pi_{j} \Rightarrow t\right\}_{k=1 \cdots o}^{j=1 \cdots m}}\left(\mathcal{D}_{1}\right)
$$

is admissible for GUL, where they set two constraints to the form of $G_{0}:$ (i) $n, m \geqslant 1$ and $\lambda_{i} \geqslant 1$ for some $1 \leqslant i \leqslant n$; (ii) $p$ does not occur in $\Gamma_{i}, \Delta_{i}, \Pi_{j}, \Sigma_{k}$ for $i=1 \cdots n, j=1 \cdots m, k=1 \cdots o$.

We may regard $\left(\mathcal{D}_{1}\right)$ as a procedure whose input and output are the premise and conclusion of $\left(\mathcal{D}_{1}\right)$, respectively. We denote the conclusion of $\left(\mathcal{D}_{1}\right)$ by $\mathcal{D}_{1}\left(G_{0}\right)$ when its premise is $G_{0}$. Observe that Metcalfe and Montagna had succeeded in defining the suitable conclusion for an almost arbitrary premise in $\left(\mathcal{D}_{1}\right)$, but it seems impossible for GIUL (see Section 3 for an example). We then define the following generalized density rule $\left(\mathcal{D}_{0}\right)$ for

\section{GL $\in\{$ GUL, GIUL, GMTL, GIMTL $\}$}

and prove its admissibility in Section 9.

Theorem 1 (Main theorem). Let $n, m \geqslant 1$, $p$ does not occur in $G^{\prime}, \Gamma_{i}, \Delta_{i}, \Pi_{j}$ or $\Sigma_{j}$ for all $1 \leqslant i \leqslant n, 1 \leqslant j \leqslant m$. Then the strong density rule

$$
\frac{G_{0} \equiv G^{\prime}\left|\left\{\Gamma_{i}, p \Rightarrow \Delta_{i}\right\}_{i=1 \cdots n}\right|\left\{\Pi_{j} \Rightarrow p, \Sigma_{j}\right\}_{j=1 \cdots m}}{\mathcal{D}_{0}\left(G_{0}\right) \equiv G^{\prime} \mid\left\{\Gamma_{i}, \Pi_{j} \Rightarrow \Delta_{i}, \Sigma_{j}\right\}_{i=1 \cdots n ; j=1 \cdots m}}\left(\mathcal{D}_{0}\right)
$$

is admissible in $\mathbf{G L}$. 
In proving the admissibility of $\left(\mathcal{D}_{1}\right)$, Metcalfe and Montagna made some restriction on the proof $\tau$ of $G_{0}$, i.e., converted $\tau$ into an r-proof. The reason why they need an r-proof is that they set the constraint (i) to $G_{0}$. We may imagine the restriction on $\tau$ and the constraints to $G_{0}$ as two pallets of a balance, i.e., one is strong if another is weak and vice versa. Observe that we select the weakest form of $G_{0}$ in $\left(\mathcal{D}_{0}\right)$ that guarantees the validity of $(D)$. Then it is natural that we need make the strongest restriction on the proof $\tau$ of $G_{0}$. But it seems extremely difficult to follow such a way to prove the admissibility of $\left(\mathcal{D}_{0}\right)$.

In order to overcome such a difficulty, we first of all choose Avron-style hypersequent calculi as the underlying systems (see Appendix A.1). Let $\tau$ be a cut-free proof of $G_{0}$ in GL. Starting with $\tau$, we construct a proof $\tau^{*}$ of $G \mid G^{*}$ in a restricted subsystem $\mathbf{G L}_{\Omega}$ of $\mathbf{G L}$ by a systematic novel manipulations in Section 4. Roughly speaking, each sequent of $G$ is a copy of some sequent of $G_{0}$, and each sequent of $G^{*}$ is a copy of some contraction sequent in $\tau$. In Section 5, we define the generalized density rule $(\mathcal{D})$ in $\mathbf{G L}_{\Omega}$ and prove that it is admissible.

Now, starting with $G \mid G^{*}$ and its proof $\tau^{*}$, we construct a proof $\tau^{\text {is }}$ of $G^{\text {出 }}$ in $\mathbf{G L}_{\Omega}$ such that each sequent of $G^{\text {t }}$ is a copy of some sequent of $G$. Then $r_{\mathrm{GL}_{\Omega}} \mathcal{D}\left(G^{\boldsymbol{t}}\right)$ by the admissibility of $(\mathcal{D})$. Then $\vdash_{G L} \mathcal{D}_{0}\left(G_{0}\right)$ by Lemma 29 . Hence the density elimination theorem holds in GL. Especially, the standard completeness of IUL follows from Theorem 62 of [9].

$G^{\text {th }}$ is constructed by eliminating $(p E C)$-sequents in $G \mid G^{*}$ one by one. In order to control the process, we introduce the set $I=\left\{H_{i_{1}}^{c}, \cdots, H_{i_{m}}^{c}\right\}$ of $(p E C)$-nodes of $\tau^{*}$ and the set $\mathbf{I}$ of the branches relative to $I$ and construct $G_{I}^{\text {is }}$ such that $G_{I}^{\text {i }}$ does not contain $(p E C)$-sequents lower than any node in $I$, i.e., $S_{j}^{c} \in G_{\mathbf{I}}^{\text {is }}$ implies $H_{j}^{c} \| H_{i}^{c}$ for all $H_{i}^{c} \in I$. The procedure is called the separation algorithm of branches in which we introduce another novel manipulation and call it derivation-grafting operation in Sections 7 and 8.

\section{Preliminaries}

In this section, we recall the basic definitions and results involved, which are mainly from [9]. Substructural fuzzy logics are based on a countable propositional language with formulas built inductively as usual from a set of propositional variables VAR, binary connectives $\odot, \rightarrow, \wedge, \vee$, and constants $\perp, T, t, f$ with definable connective $\neg A:=A \rightarrow f$.

Definition 1. $([9,12])$ A sequent is an ordered pair $(\Gamma, \Delta)$ of finite multisets (possibly empty) of formulas, which we denote by $\Gamma \Rightarrow \Delta$. $\Gamma$ and $\Delta$ are called the antecedent and succedents, respectively, of the sequent and each formula in $\Gamma$ and $\Delta$ is called a sequent-formula. A hypersequent $G$ is a finite multiset of the form $\Gamma_{1} \Rightarrow \Delta_{1}|\cdots| \Gamma_{n} \Rightarrow \Delta_{n}$, where each $\Gamma_{i} \Rightarrow \Delta_{i}$ is a sequent and is called a component of $G$ for each $1 \leqslant i \leqslant n$. If $\Delta_{i}$ contains at most one formula for $i=1 \cdots n$, then the hypersequent is single-conclusion, otherwise it is a multiple-conclusion.

Definition 2. Let $S$ be a sequent and $G=S_{1}|\cdots| S_{m}$ a hypersequent. We say that $S \in G$ if $S$ is one of $S_{1}, \cdots, S_{m}$.

Notation 1. Let $G_{1}$ and $G_{2}$ be two hypersequents. We will assume from now on that all set terminology refers to multisets, adopting the conventions of writing $\Gamma, \Delta$ for the multiset union of $\Gamma$ and $\Delta, A$ for the singleton multiset $\{A\}$, and $\lambda \Gamma$ for the multiset union of $\lambda$ copies of $\Gamma$ for $\lambda \in \mathbf{N}$. By $G_{1} \subseteq G_{2}$ we mean that $S \in G_{2}$ for all $S \in G_{1}$ and the multiplicity of $S$ in $G_{1}$ is not more than that of $S$ in $G_{2}$. We will use $G_{1}=G_{2}, G_{1} \cap G_{2}$, $G_{1} \cup G_{2}, G_{1} \backslash G_{2}$ by their standard meaning for multisets by default and we will declare when we use them for sets. We sometimes write $S_{1}|\cdots| S_{m}$ and $G \mid \overbrace{S|\cdots| S}^{n \text { copies }}$ as $\left\{S_{1}, \cdots, S_{m}\right\}, G \mid S^{n}\left(\right.$ or $\left.G \mid\{S\}^{n}\right)$, respectively.

Definition 3. ([12]) A hypersequent rule is an ordered pair consisting of a sequence of hypersequents $G_{1}, \cdots, G_{n}$ called the premises (upper hypersequents) of the rule, and a hypersequent $G$ called the conclusion 
(lower hypersequent), written by $\frac{G_{1} \cdots G_{n}}{G}$. If $n=0$, then the rule has no premise and is called an initial sequent. The single-conclusion version of a rule adds the restriction that both the premises and conclusion must be single-conclusion; otherwise the rule is multiple-conclusion.

Definition 4. ([9]) GUL and GIUL consist of the single-conclusion and multiple-conclusion versions of the following initial sequents and rules, respectively:

Initial sequents

$$
\overline{A \Rightarrow A}^{(I D)} \overline{\Gamma \Rightarrow \mathrm{T}, \Delta}\left(\mathrm{T}_{r}\right) \quad \overline{\Gamma, \perp \Rightarrow \Delta}\left(\perp_{l}\right) \quad \overline{\Rightarrow t}\left(t_{r}\right) \quad \overline{f \Rightarrow}\left(f_{l}\right)
$$

Structural rules

$$
\begin{gathered}
\frac{G|\Gamma \Rightarrow A| \Gamma \Rightarrow A}{G \mid \Gamma \Rightarrow A}(E C) \quad \frac{G}{G \mid \Gamma \Rightarrow A}(E W) \\
\frac{G_{1}\left|\Gamma_{1}, \Pi_{1} \Rightarrow \Sigma_{1}, \Delta_{1} \quad G_{2}\right| \Gamma_{2}, \Pi_{2} \Rightarrow \Sigma_{2}, \Delta_{2}}{G_{1}\left|G_{2}\right| \Gamma_{1}, \Gamma_{2} \Rightarrow \Delta_{1}, \Delta_{2} \mid \Pi_{1}, \Pi_{2} \Rightarrow \Sigma_{1}, \Sigma_{2}}(C O M)
\end{gathered}
$$

Logical rules

$$
\begin{aligned}
& \frac{G \mid \Gamma \Rightarrow \Delta}{G \mid \Gamma, t, \Rightarrow \Delta}\left(t_{l}\right) \\
& \frac{G_{1}\left|\Gamma_{1} \Rightarrow A, \Delta_{1} \quad G_{2}\right| \Gamma_{2}, B \Rightarrow \Delta_{2}}{G_{1}\left|G_{2}\right| \Gamma_{1}, \Gamma_{2}, A \rightarrow B \Rightarrow \Delta_{1}, \Delta_{2}}\left(\rightarrow_{l}\right) \\
& \frac{G \mid \Gamma, A, B \Rightarrow \Delta}{G \mid \Gamma, A \odot B \Rightarrow \Delta}\left(\odot_{l}\right) \\
& \frac{G \mid \Gamma, A \Rightarrow \Delta}{G \mid \Gamma, A \wedge B \Rightarrow \Delta}\left(\wedge_{l r}\right) \\
& \frac{G_{1}\left|\Gamma \Rightarrow A, \Delta \quad G_{2}\right| \Gamma \Rightarrow B, \Delta}{G_{1}\left|G_{2}\right| \Gamma \Rightarrow A \wedge B, \Delta}\left(\wedge_{r}\right) \\
& \frac{G \mid \Gamma \Rightarrow B, \Delta}{G \mid \Gamma \Rightarrow A \vee B, \Delta}\left(\vee_{r l}\right) \\
& \begin{array}{r}
\frac{G \mid \Gamma \Rightarrow \Delta}{G \mid \Gamma \Rightarrow f, \Delta}\left(f_{r}\right) \\
\frac{G \mid \Gamma, A \Rightarrow B, \Delta}{G \mid \Gamma \Rightarrow A \rightarrow B, \Delta}\left(\rightarrow_{r}\right) \\
\frac{G_{1}\left|\Gamma_{1} \Rightarrow A, \Delta_{1} G_{2}\right| \Gamma_{2} \Rightarrow B, \Delta_{2}}{G_{1}\left|G_{2}\right| \Gamma_{1}, \Gamma_{2} \Rightarrow A \odot B, \Delta_{1}, \Delta_{2}}\left(\odot_{r}\right) \\
\frac{G \mid \Gamma, B \Rightarrow \Delta}{G \mid \Gamma, A \wedge B \Rightarrow \Delta}\left(\wedge_{l l}\right) \\
\frac{G \mid \Gamma \Rightarrow A, \Delta}{G \mid \Gamma \Rightarrow A \vee B, \Delta}\left(\vee_{r r}\right) \\
\frac{G_{1}\left|\Gamma, A \Rightarrow \Delta \quad G_{2}\right| \Gamma, B \Rightarrow \Delta}{G_{1}\left|G_{2}\right| \Gamma, A \vee B \Rightarrow \Delta}\left(\vee_{l}\right)
\end{array}
\end{aligned}
$$

Cut rule

$$
\frac{G_{1}\left|\Gamma_{1}, A \Rightarrow \Delta_{1} \quad G_{2}\right| \Gamma_{2} \Rightarrow A, \Delta_{2}}{G_{1}\left|G_{2}\right| \Gamma_{1}, \Gamma_{2} \Rightarrow \Delta_{1}, \Delta_{2}}(\text { CUT })
$$

Definition 5. ([9]) GMTL and GIMTL are GUL and GIUL plus the single conclusion and multiple-conclusion versions, respectively, of:

$$
\frac{G \mid \Gamma \Rightarrow \Delta}{G \mid \Gamma, A \Rightarrow \Delta}(W L), \frac{G \mid \Gamma \Rightarrow \Delta}{G \mid \Gamma \Rightarrow A, \Delta}(W R)
$$

Definition 6. (i) $(I) \in\left\{\left(t_{l}\right),\left(f_{r}\right),\left(\rightarrow_{r}\right),\left(\odot_{l}\right),\left(\wedge_{l r}\right),\left(\wedge_{l l}\right),\left(\vee_{r r}\right),\left(\vee_{r l}\right),(W L),(W R)\right\}$ and $(I I) \in\left\{\left(\rightarrow_{l}\right),\left(\odot_{r}\right),\left(\wedge_{r}\right),\left(\vee_{l}\right),(C O M)\right\}$;

(ii) By $\frac{G^{\prime}\left|S^{\prime} \quad G^{\prime \prime}\right| S^{\prime \prime}}{G^{\prime}\left|G^{\prime \prime}\right| H^{\prime}}(I I)$ (or $\left.\frac{G^{\prime} \mid S^{\prime}}{G^{\prime} \mid H^{\prime}}(I)\right)$ we denote an instance of a two-premise rule (II) (or one-premise rule $(I))$ of $\mathbf{G} \mathbf{L}$, where $S^{\prime}$ and $S^{\prime \prime}$ are its focus sequents and $H^{\prime}$ is its principle sequent $\left(\right.$ for $\left(\rightarrow_{l}\right),\left(\odot_{r}\right),\left(\wedge_{r}\right)$ and $\left.\left(\vee_{l}\right)\right)$ or hypersequent $\left(\right.$ for $(C O M),\left(\wedge_{r w}\right)$ and $\left(\vee_{l w}\right)$, see Definition 12$)$. 
Definition 7. ([9]) $\mathbf{G L}^{\mathrm{D}}$ is $\mathbf{G L}$ extended with the following density rule:

$$
\frac{G\left|\Gamma_{1}, p \Rightarrow \Delta_{1}\right| \Gamma_{2} \Rightarrow p, \Delta_{2}}{G \mid \Gamma_{1}, \Gamma_{2} \Rightarrow \Delta_{1}, \Delta_{2}}(D)
$$

where $p$ does not occur in $G, \Gamma_{1}, \Gamma_{2}, \Delta_{1}$ or $\Delta_{2}$.

Definition 8. ([12]) A derivation $\tau$ of a hypersequent $G$ from hypersequents $G_{1}, \cdots, G_{n}$ in a hypersequent calculus $\mathbf{G L}$ is a labeled tree with the root labeled by $G$, leaves labeled initial sequents or some $G_{1}, \cdots, G_{n}$, and for each node labeled $G_{0}^{\prime}$ with parent nodes labeled $G_{1}^{\prime}, \cdots, G_{m}^{\prime}$ (where possibly $\left.m=0\right), \frac{G_{1}^{\prime} \cdots G_{m}^{\prime}}{G_{0}^{\prime}}$ is an instance of a rule of $\mathbf{G L}$.

Notation 2. (i) $\frac{G_{1} \cdots G_{n}}{G_{0}}\langle\tau\rangle$ denotes that $\tau$ is a derivation of $G_{0}$ from $G_{1}, \cdots, G_{n}$;

(ii) Let $H$ be a hypersequent. $H \in \tau$ denotes that $H$ is a node of $\tau$. We call $H$ a leaf hypersequent if $H$ is a leaf of $\tau$, the root hypersequent if it is the root of $\tau$. $\frac{G_{1}^{\prime} \cdots G_{m}^{\prime}}{G_{0}^{\prime}} \in \tau$ denotes that $G_{0}^{\prime} \in \tau$ and its parent nodes are $G_{1}^{\prime}, \cdots, G_{m}^{\prime} ;$

(iii) Let $H \in \tau$ then $\tau(H)$ denotes the subtree of $\tau$ rooted at $H$;

(iv) $\tau$ determines a partial order $\leqslant \tau$ with the root as the least element. $H_{1} \| H_{2}$ denotes $H_{1} \nless \tau H_{2}$ and $H_{2} \nless \tau H_{1}$ for any $H_{1}, H_{2} \in \tau$. By $H_{1}=\tau H_{2}$ we mean that $H_{1}$ is the same node as $H_{2}$ in $\tau$. We sometimes write $\leqslant \tau$ as $\leqslant$

(v) An inference of the form $\frac{G^{\prime} \mid S^{n}}{G^{\prime} \mid S} \in \tau$ is called the full external contraction and denoted by $\left(E C^{*}\right)$, if $n \geqslant 2$, $G^{\prime} \mid S^{n}$ is not a lower hypersequent of an application of (EC) whose contraction sequent is $S$, and $G^{\prime} \mid S$ not an upper one in $\tau$.

Definition 9. Let $\tau$ be a derivation of $G$ and $H \in \tau$. The thread $T h_{\tau}(H)$ of $\tau$ at $H$ is a sequence $H_{0}, \cdots, H_{n}$ of node hypersequents of $\tau$ such that $H_{0}={ }_{\tau} H, H_{n}={ }_{\tau} G, \frac{H_{k}}{H_{k+1}} \in \tau$ or there exists $G^{\prime} \in \tau$ such that $\frac{H_{k} G^{\prime}}{H_{k+1}}$ or $\frac{G^{\prime} \quad H_{k}}{H_{k+1}}$ in $\tau$ for all $0 \leqslant k \leqslant n-1$.

Proposition 1. Let $H_{1}, H_{2} \in \tau$. Then

(i) $H_{1} \leqslant H_{2}$ if and only if $H_{1} \in T h_{\tau}\left(H_{2}\right)$;

(ii) $H_{1} \| H_{2}$ and $H_{1} \leqslant H_{3}$ imply $H_{2} \| H_{3}$;

(iii) $H_{1} \leqslant H_{3}$ and $H_{2} \leqslant H_{3}$ imply $H_{1} H H_{2}$.

We need the following definition to give each node of $\tau$ an identification number, which is used in Construction 3 to differentiate sequents in a hypersequent in a proof.

Definition 10. (Appendix A.5.2) Let $H \in \tau$ and $T h(H)=\left(H_{0}, \cdots, H_{n}\right)$. Let $b_{n}:=1$,

$$
b_{k}:= \begin{cases}1 & \text { if } \frac{G^{\prime} H_{k}}{H_{k+1}} \in \tau, \\ 0 & \text { if } \frac{H_{k}}{H_{k+1}} \in \tau \text { or } \frac{H_{k} G^{\prime}}{H_{k+1}} \in \tau\end{cases}
$$

for all $0 \leqslant k \leqslant n-1$. Then $\mathcal{P}(H):=\sum_{k=0}^{k=n} 2^{k} b_{k}$ and call it the position of $H$ in $\tau$. 
Definition 11. A rule is admissible for a calculus $\mathbf{G L}$ if whenever its premises are derivable in $\mathbf{G L}$, then so is its conclusion.

Lemma 1. ([9]) Cut-elimination holds for GL, i.e., proofs using (CUT) can be transformed syntactically into proofs not using (CUT).

\section{Proof of the Main Theorem: A Computational Example}

In this section, we present an example to illustrate the proof of the main theorem.

Let $G_{0} \equiv \Rightarrow p, B|B \Rightarrow p, \neg A \odot \neg A| p \Rightarrow C \mid C, p \Rightarrow A \odot A$. $G_{0}$ is a theorem of IUL and a cut-free proof $\tau$ of $G_{0}$ is shown in Figure 1, where we use an additional rule $\frac{\Gamma, A \Rightarrow \Delta}{\Gamma \Rightarrow \neg A, \Delta}(\neg r)$ for simplicity. Note that we denote three applications of $(E C)$ in $\tau$ respectively by $(E C)_{1},(E C)_{2},(E C)_{3}$ and three $\left(\odot_{r}\right)$ by $\left(\odot_{r}\right)_{1},\left(\odot_{r}\right)_{2}$ and $\left(\odot_{r}\right)_{3}$.

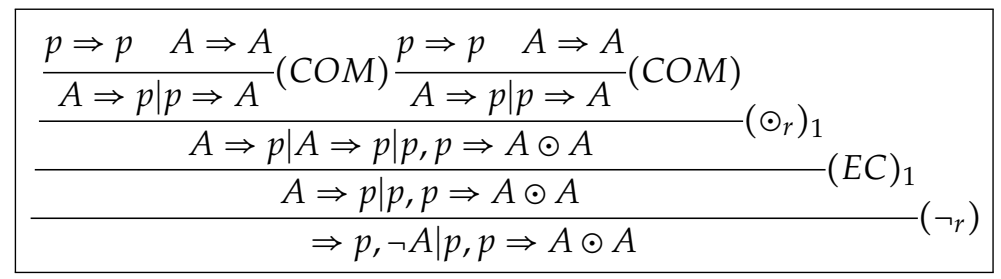

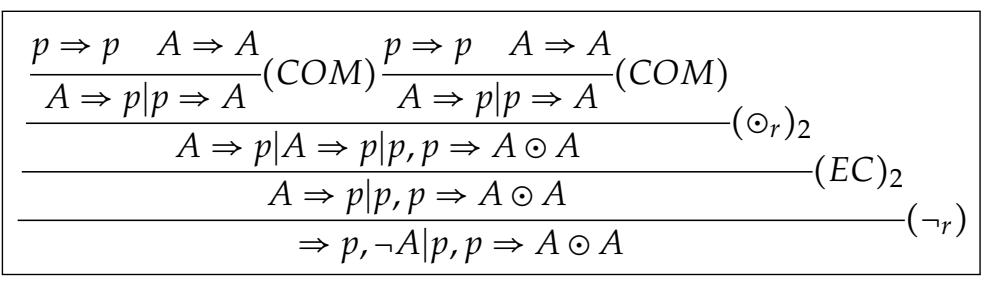

(continued)

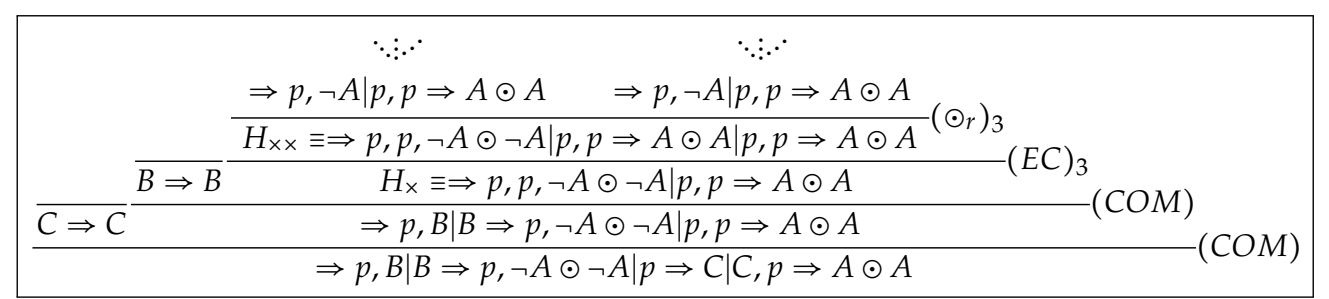

Figure 1. A proof $\tau$ of $G_{0}$.

By applying (D) to free combinations of all sequents in $\Rightarrow p, B \mid B \Rightarrow p, \neg A \odot \neg A$ and in $p \Rightarrow$ $C \mid C, p \Rightarrow A \odot A$, we get that $H_{0} \equiv \Rightarrow B, C|C \Rightarrow A \odot A, B| B \Rightarrow C, \neg A \odot \neg A \mid C, B \Rightarrow A \odot A, \neg A \odot \neg A$. $H_{0}$ is a theorem of IUL and a cut-free proof $\rho$ of $H_{0}$ is shown in Figure 2. It supports the validity of the generalized density rule $\left(\mathcal{D}_{0}\right)$ in Section 1 , as an instance of $\left(\mathcal{D}_{0}\right)$. 


\begin{aligned} &$\frac{\frac{A \Rightarrow A \quad A \Rightarrow A}{A, A \Rightarrow A \odot A}}{\frac{A \Rightarrow \neg A, A \odot A}{A, A \Rightarrow \neg A \odot \neg A, A \odot A, A \odot A}} \frac{A \Rightarrow A \quad A \Rightarrow A}{A, A \Rightarrow A \odot A} \\ & \frac{\mathrm{C \Rightarrow C}}{\frac{B \Rightarrow B}{\frac{A \Rightarrow A, A \odot A}{A, B \Rightarrow A \odot A, \neg A \odot \neg A \mid A \Rightarrow A \odot A, B}}} \\ &$\hline$H_{1} \equiv A \Rightarrow C|A, B \Rightarrow A \odot A, \neg A \odot \neg A| C \Rightarrow A \odot A, B\end{aligned}$

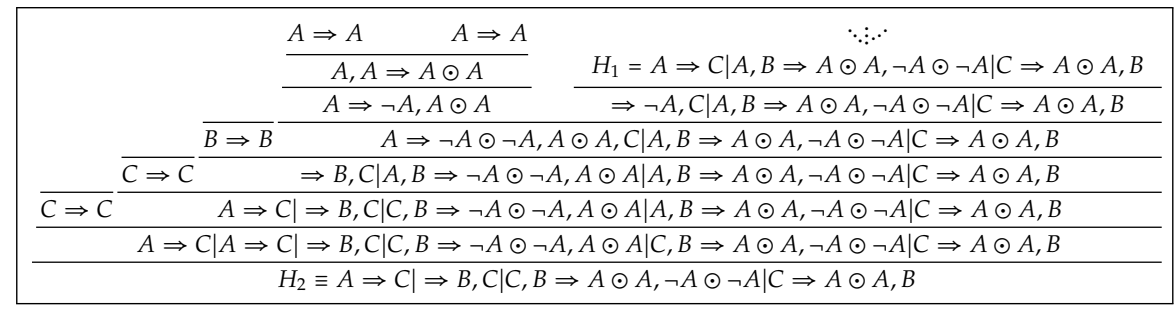

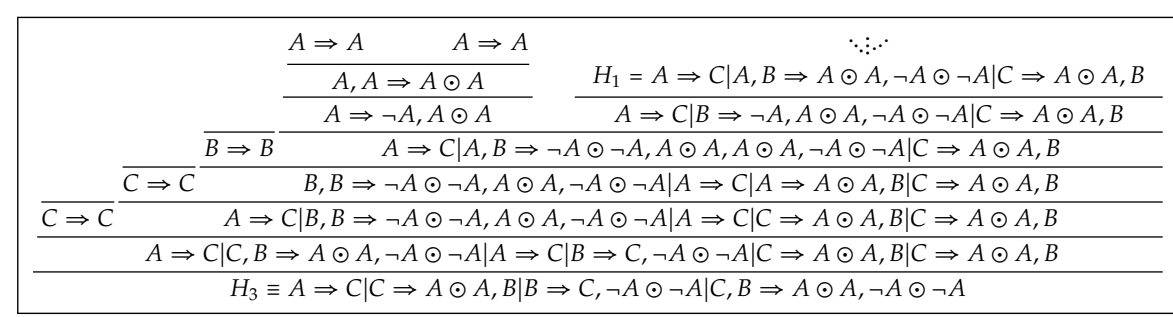

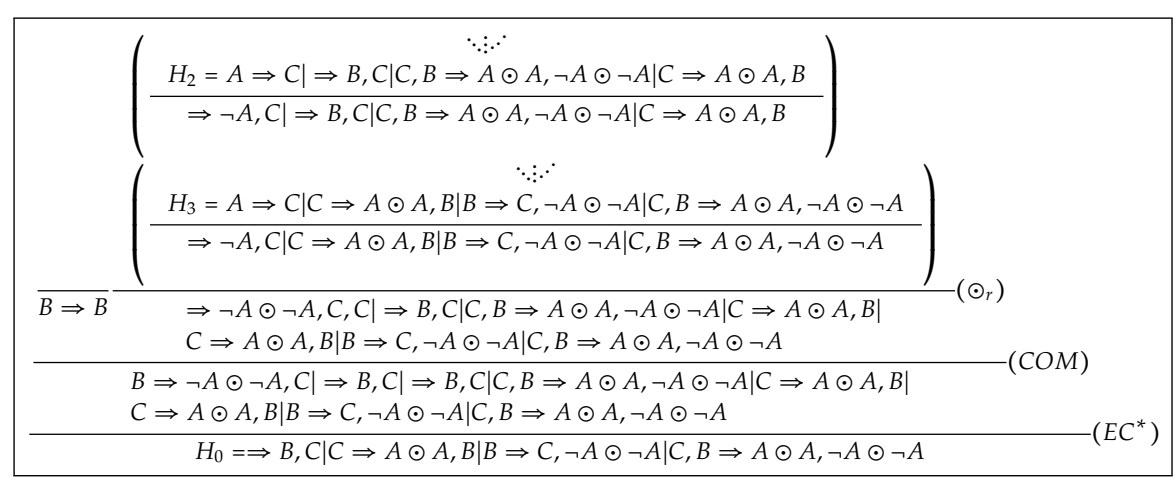

Figure 2. A proof $\rho$ of $H_{0}$.

Our task is to construct $\rho$, starting from $\tau$. The tree structure of $\rho$ is more complicated than that of $\tau$. Compared with UL, MTL and IMTL, there is no one-to-one correspondence between nodes in $\tau$ and $\rho$.

Following the method given by G. Metcalfe and F. Montagna, we need to define a generalized density rule for IUL. We denote such an expected unknown rule by $\left(\mathcal{D}_{x}\right)$ for convenience. Then $\mathcal{D}_{x}(H)$ must be definable for all $H \in \tau$. Naturally,

$$
\begin{gathered}
\mathcal{D}_{x}(p \Rightarrow p)=\Rightarrow t ; \\
\mathcal{D}_{x}(A \Rightarrow p \mid p \Rightarrow A)=A \Rightarrow A ; \\
\mathcal{D}_{x}(\Rightarrow p, \neg A \mid p, p \Rightarrow A \odot A)=\Rightarrow \neg A, \neg A, A \odot A ; \\
\mathcal{D}_{x}(\Rightarrow p, B|B \Rightarrow p, \neg A \odot \neg A| p, p \Rightarrow A \odot A)= \\
\Rightarrow B, B, A \odot A|B, B \Rightarrow A \odot A, \neg A \odot \neg A, \neg A \odot \neg A| B \Rightarrow A \odot A, B, \neg A \odot \neg A ; \\
\mathcal{D}_{x}\left(G_{0}\right)=\mathcal{D}_{0}\left(G_{0}\right)=H_{0} .
\end{gathered}
$$


However, we could not find a suitable way to define $\mathcal{D}_{x}\left(H_{\times \times}\right)$and $\mathcal{D}_{x}\left(H_{\times}\right)$for $H_{\times \times}$and $H_{\times}$in $\tau$, see Figure 1 . This is the biggest difficulty we encounter in the case of IUL such that it is hard to prove density elimination for IUL. A possible way is to define $\mathcal{D}_{x}(\Rightarrow p, p, \neg A \odot \neg A \mid p, p \Rightarrow A \odot A)$ as $\Rightarrow t, A \odot A, \neg A \odot \neg A$. Unfortunately, it is not a theorem of IUL.

Notice that two upper hypersequents $\Rightarrow p, \neg A \mid p, p \Rightarrow A \odot A$ of $\left(\odot_{r}\right)_{3}$ are permissible inputs of $\left(\mathcal{D}_{x}\right)$. Why is $H_{\times \times}$an invalid input? One reason is that, two applications $(E C)_{1}$ and $(E C)_{2}$ cut off two sequents $A \Rightarrow p$ such that two $p^{\prime} s$ disappear in all nodes lower than upper hypersequent of $(E C)_{1}$ or $(E C)_{2}$, including $H_{x \times}$. These make occurrences of $p^{\prime} s$ to be incomplete in $H_{x \times}$. We then perform the following operation in order to get complete occurrences of $p^{\prime} s$ in $H_{x \times}$.

Step 1 (preprocessing of $\tau$ ). Firstly, we replace $H$ with $H \mid S^{\prime}$ for all $\frac{G^{\prime}\left|S^{\prime}\right| S^{\prime}}{G^{\prime} \mid S^{\prime}}(E C)_{k} \in \tau, H \leqslant G^{\prime} \mid S^{\prime}$ then replace $\frac{G^{\prime}\left|S^{\prime}\right| S^{\prime}}{G^{\prime}\left|S^{\prime}\right| S^{\prime}}(E C)_{k}$ with $G^{\prime}\left|S^{\prime}\right| S^{\prime}$ for all $k=1,2,3$. Then we construct a proof without $(E C)$, which we denote by $\tau_{1}$, as shown in Figure 3. We call such manipulations sequent-inserting operations, which eliminate applications of $(E C)$ in $\tau$.

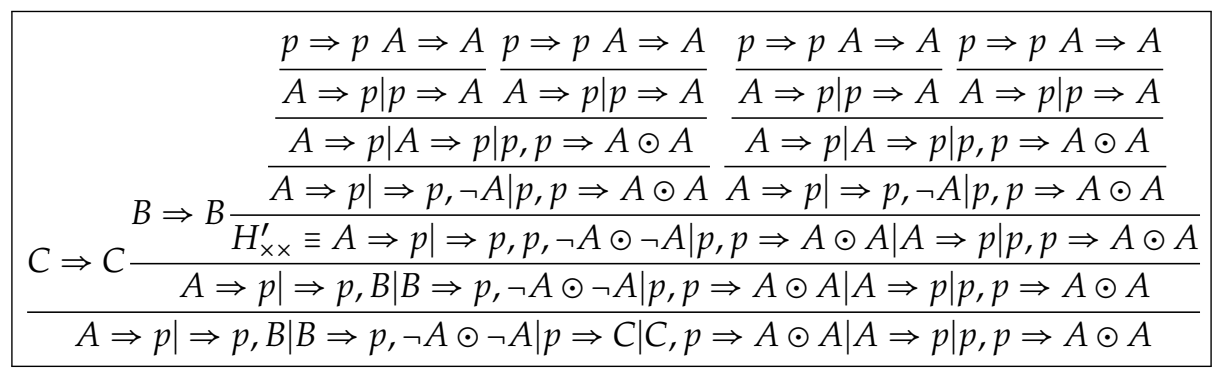

Figure 3. A proof $\tau_{1}$.

However, we also cannot define $\mathcal{D}_{x}\left(H_{\times x}^{\prime}\right)$ for $H_{\times x}^{\prime} \in \tau_{1}$ in that $\Rightarrow p, p, \neg A \odot \neg A \mid p, p \Rightarrow A \odot A \subseteq H_{\times x}^{\prime}$. The reason is that the origins of $p^{\prime} s$ in $H_{x \times}^{\prime}$ are indistinguishable if we regard all leaves in the form $p \Rightarrow p$ as the origins of $p^{\prime} s$ which occur in the inner node. For example, we do not know which $p$ comes from the left subtree of $\tau_{1}\left(H_{\times x}^{\prime}\right)$ and which from the right subtree in two occurrences of $p^{\prime} s$ in $\Rightarrow p, p, \neg A \odot \neg A \in H_{\times x}^{\prime}$. We then perform the following operation in order to make all occurrences of $p^{\prime} s$ in $H_{x \times}^{\prime}$ distinguishable.

We assign the unique identification number to each leaf in the form $p \Rightarrow p \in \tau_{1}$ and transfer these identification numbers from leaves to the root, as shown in Figure 4. We denote the proof of $G \mid G^{*}$ resulting from this step by $\tau^{*}$, where $G \equiv \Rightarrow p_{2}, B\left|B \Rightarrow p_{4}, \neg A \odot \neg A\right| p_{1} \Rightarrow C \mid C, p_{2} \Rightarrow A \odot A$ in which each sequent is a copy of some sequent in $G_{0}$ and $G^{*} \equiv A \Rightarrow p_{1}\left|A \Rightarrow p_{3}\right| p_{3}, p_{4} \Rightarrow A \odot A$ in which each sequent is a copy of some external contraction sequent in (EC)-node of $\tau$. We call such manipulations eigenvariable-labeling operations, which make us to trace eigenvariables in $\tau$.

\begin{tabular}{|c|c|}
\hline$\frac{p_{1} \Rightarrow p_{1} A \Rightarrow A}{A \Rightarrow p_{1} \mid p_{1} \Rightarrow A} \frac{p_{2} \Rightarrow p_{2} A \Rightarrow A}{A \Rightarrow p_{2} \mid p_{2} \Rightarrow A}$ & $\frac{p_{3} \Rightarrow p_{3} A \Rightarrow A}{A \Rightarrow p_{3} \mid p_{3} \Rightarrow A} \frac{p_{4} \Rightarrow p_{4} A \Rightarrow A}{A \Rightarrow p_{4} \mid p_{4} \Rightarrow A}$ \\
\hline \multicolumn{2}{|c|}{ 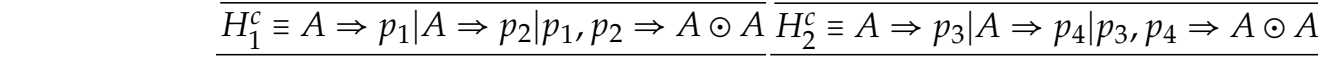 } \\
\hline$B \Rightarrow B \stackrel{A \Rightarrow p_{1}\left|\Rightarrow p_{2}, \neg A\right| p_{1}, p_{2} \Rightarrow A \odot A}{A}$ & $A \Rightarrow p_{3}\left|\Rightarrow p_{4}, \neg A\right| p_{3}, p_{4} \Rightarrow A \odot A$ \\
\hline \multicolumn{2}{|c|}{ 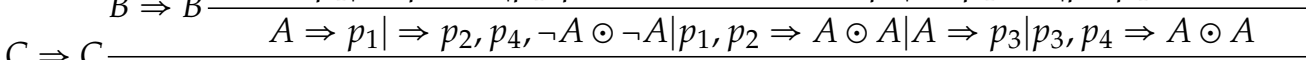 } \\
\hline \multicolumn{2}{|c|}{$C \Rightarrow C H_{3}^{c} \equiv A \Rightarrow p_{1}\left|\Rightarrow p_{2}, B\right| B \Rightarrow p_{4}, \neg A \odot \neg A\left|p_{1}, p_{2} \Rightarrow A \odot A\right| A \Rightarrow p_{3} \mid p_{3}, p_{4} \Rightarrow A \odot A$} \\
\hline \multicolumn{2}{|c|}{$A \Rightarrow p_{1}\left|\Rightarrow p_{2}, B\right| B \Rightarrow p_{4}, \neg A \odot \neg A\left|p_{1} \Rightarrow C\right| C, p_{2} \Rightarrow A \odot A\left|A \Rightarrow p_{3}\right| p_{3}, p_{4} \Rightarrow A \odot A$} \\
\hline
\end{tabular}

Figure 4. A proof $\tau^{*}$ of $G \mid G^{*}$. 
Then all occurrences of $p$ in $\tau^{*}$ are distinguishable and we regard them as distinct eigenvariables (See Definition 18 (i)). Firstly, by selecting $p_{1}$ as the eigenvariable and applying $(D)$ to $G \mid G^{*}$, we get

$$
G^{\prime} \equiv A \Rightarrow C\left|\Rightarrow p_{2}, B\right| B \Rightarrow p_{4}, \neg A \odot \neg A\left|C, p_{2} \Rightarrow A \odot A\right| A \Rightarrow p_{3} \mid p_{3}, p_{4} \Rightarrow A \odot A .
$$

Secondly, by selecting $p_{2}$ and applying $(D)$ to $G^{\prime}$, we get

$$
G^{\prime \prime} \equiv A \Rightarrow C\left|B \Rightarrow p_{4}, \neg A \odot \neg A\right| C \Rightarrow B, A \odot A\left|A \Rightarrow p_{3}\right| p_{3}, p_{4} \Rightarrow A \odot A .
$$

Repeatedly, we get

$$
G^{\prime \prime \prime \prime} \equiv A \Rightarrow C|A, B \Rightarrow A \odot A, \neg A \odot \neg A| C \Rightarrow A \odot A, B .
$$

We define such iterative applications of $(D)$ as $\mathcal{D}$-rule (See Definition 20). Lemma 10 shows that $\vdash_{\text {GIUL }} \mathcal{D}\left(G \mid G^{*}\right)$ if $\vdash_{\text {GIUL }} G \mid G^{*}$. Then we obtain $\vdash_{\text {GIUL }} \mathcal{D}\left(G \mid G^{*}\right)$, i.e., $\vdash_{\text {GIUL }} G^{\prime \prime \prime \prime}$.

A miracle happens here! The difficulty that we encountered in GIUL is overcome by converting $H_{\times x}^{\prime}=A \Rightarrow p|\Rightarrow p, p, \neg A \odot \neg A| p, p \Rightarrow A \odot A|A \Rightarrow p| p, p \Rightarrow A \odot A$ into $A \Rightarrow p_{1} \mid \Rightarrow p_{2}, p_{4}, \neg A \odot$ $\neg A\left|p_{1}, p_{2} \Rightarrow A \odot A\right| A \Rightarrow p_{3} \mid p_{3}, p_{4} \Rightarrow A \odot A$ and using $(\mathcal{D})$ to replace $\left(\mathcal{D}_{x}\right)$.

Why do we assign the unique identification number to each $p \Rightarrow p \in \tau_{1}$ ? We would return back to the same situation as that of $\tau_{1}$ if we assign the same indices to all $p \Rightarrow p \in \tau_{1}$ or, replace $p_{3} \Rightarrow p_{3}$ and $p_{4} \Rightarrow p_{4}$ by $p_{2} \Rightarrow p_{2}$ in $\tau^{*}$.

Note that $\mathcal{D}\left(G \mid G^{*}\right)=H_{1}$. So we have built up a one-one correspondence between the proof $\tau^{*}$ of $G \mid G^{*}$ and that of $H_{1}$. Observe that each sequent in $G^{*}$ is not a copy of any sequent in $G_{0}$. In the following steps, we work on eliminating these sequents in $G^{*}$.

Step 2 (extraction of elimination rules). We select $A \Rightarrow p_{2}$ as the focus sequent in $H_{1}^{c}$ in $\tau^{*}$ and keep $A \Rightarrow p_{1}$ unchanged from $H_{1}^{c}$ downward to $G \mid G^{*}$ (See Figure 4). So we extract a derivation from $A \Rightarrow p_{2}$ by pruning some sequents (or hypersequents) in $\tau^{*}$, which we denote by $\tau_{H_{1}^{c}: A \Rightarrow p_{2}}^{*}$ as shown in Figure 5.

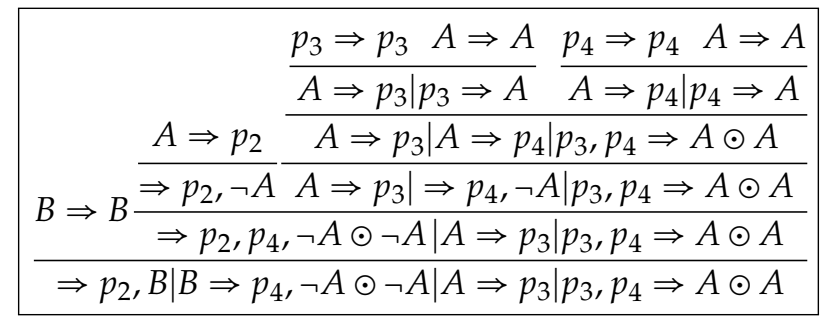

Figure 5. A derivation $\tau_{H_{1}^{c}: A \Rightarrow p_{2}}^{*}$ from $A \Rightarrow p_{2}$.

A derivation $\tau_{H_{1}^{c}: A \Rightarrow p_{1}}^{*}$ from $A \Rightarrow p_{1}$ is constructed by replacing $p_{2}$ with $p_{1}, p_{3}$ with $p_{5}$ and $p_{4}$ with $p_{6}$ in $\tau_{H_{1}^{c}: A \Rightarrow p_{2}}^{*}$ as shown in Figure 6. Notice that we assign new identification numbers to new occurrences of $p$ in $\tau_{H_{1}^{c}: A \Rightarrow p_{1}}^{*}$. 


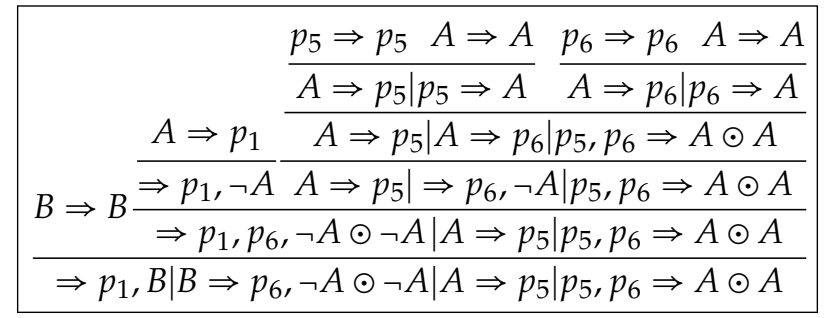

Figure 6. A derivation $\tau_{H_{1}^{c}: A \Rightarrow p_{1}}^{*}$ from $A \Rightarrow p_{1}$.

Next, we apply $\tau_{H_{1}^{c}: A \Rightarrow p_{1}}^{*}$ to $A \Rightarrow p_{1}$ in $G \mid G^{*}$. Then we construct a proof $\tau_{H_{1}^{c}: G \mid G^{*}}^{i(1)}$, as shown in Figure 7, where $G^{\prime} \equiv G \mid G^{*} \backslash\left\{A \Rightarrow p_{1}\right\}$.

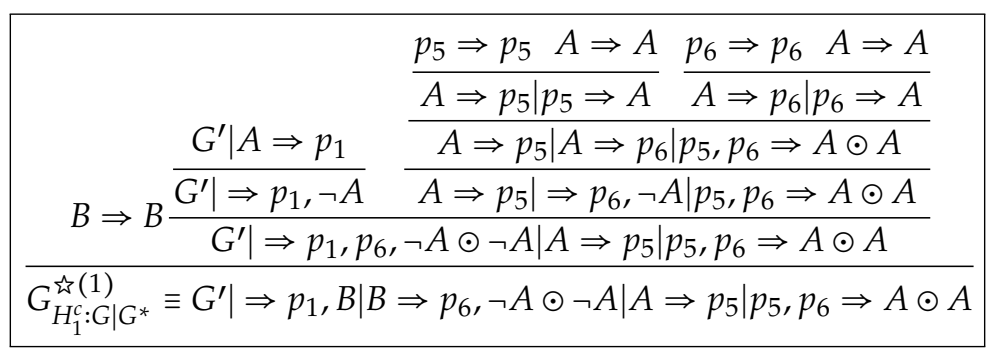

Figure 7. A proof $\tau_{H_{1}^{c}: G \mid G^{*}}^{\xi(1)}$ of $G_{H_{1}^{c}: G \mid G^{*}}^{\xi(1)}$.

However, $G_{H_{1}^{c}: G \mid G^{*}}^{i \sim(1)}=\Rightarrow p_{2}, B\left|B \Rightarrow p_{4}, \neg A \odot \neg A\right| p_{1} \Rightarrow C\left|C, p_{2} \Rightarrow A \odot A\right| A \Rightarrow p_{3}\left|p_{3}, p_{4} \Rightarrow A \odot A\right| \Rightarrow$ $p_{1}, B\left|B \Rightarrow p_{6}, \neg A \odot \neg A\right| A \Rightarrow p_{5} \mid p_{5}, p_{6} \Rightarrow A \odot A$ contains more copies of sequents from $G^{*}$ and seems more complex than $G \mid G^{*}$. We will present a unified method to tackle with it in the following steps. Other derivations are shown in Figures 8-11.

$$
\begin{aligned}
& A \Rightarrow p_{1}\left|\Rightarrow p_{2}, \neg A\right| p_{1}, p_{2} \Rightarrow A \odot A \frac{A \Rightarrow p_{4}}{\Rightarrow p_{4}, \neg A} \\
& \frac{B \Rightarrow B \frac{B}{A \Rightarrow p_{1}\left|\Rightarrow p_{2}, p_{4}, \neg A \odot \neg A\right| p_{1}, p_{2} \Rightarrow A \odot A}}{A \Rightarrow p_{1}\left|\Rightarrow p_{2}, B\right| B \Rightarrow p_{4}, \neg A \odot \neg A \mid p_{1}, p_{2} \Rightarrow A \odot A} \\
& \hline A \Rightarrow p_{1}\left|\Rightarrow p_{2}, B\right| B \Rightarrow p_{4}, \neg A \odot \neg A\left|p_{1} \Rightarrow C\right| C, p_{2} \Rightarrow A \odot A
\end{aligned}
$$

Figure 8. A derivation $\tau_{H_{2}^{c}}^{*} A \Rightarrow p_{4}$ from $A \Rightarrow p_{4}$.

$$
\begin{aligned}
& C \Rightarrow p_{5}\left|\Rightarrow p_{6}, \neg A\right| p_{5}, p_{6} \Rightarrow A \odot A \frac{A \Rightarrow p_{3}}{\Rightarrow p_{3,} A} \\
& \frac{B \Rightarrow B \frac{A}{A \Rightarrow p_{5}\left|\Rightarrow p_{6}, p_{3}, \neg A \odot \neg A\right| p_{5}, p_{6} \Rightarrow A \odot A}}{A \Rightarrow p_{5}\left|\Rightarrow p_{6}, B\right| B \Rightarrow p_{3}, \neg A \odot \neg A \mid p_{5}, p_{6} \Rightarrow A \odot A} \\
& A \Rightarrow p_{5}\left|\Rightarrow p_{6}, B\right| B \Rightarrow p_{3}, \neg A \odot \neg A\left|p_{5} \Rightarrow C\right| C, p_{6} \Rightarrow A \odot A
\end{aligned}
$$

Figure 9. A derivation $\tau_{H_{2}^{c}: A \Rightarrow p_{3}}^{*}$ from $A \Rightarrow p_{3}$. 


$$
\begin{array}{|c}
B \Rightarrow B \frac{\frac{A \Rightarrow p_{2}}{\Rightarrow p_{2}, \neg A} \frac{A \Rightarrow p_{4}}{\Rightarrow p_{2}, p_{4}, \neg A \odot \neg A}}{\Rightarrow p_{4} \neg A} \\
\Rightarrow p_{2}, B \mid B \Rightarrow p_{4}, \neg A \odot \neg A
\end{array} \quad \begin{array}{r|}
\frac{A \Rightarrow p_{5}}{\Rightarrow p_{5}, \neg A} \frac{A \Rightarrow p_{3}}{\Rightarrow p_{3}, \neg A} \\
\Rightarrow p_{5}, p_{3}, \neg A \odot \neg A \\
\hline p_{5}, B \mid B \Rightarrow p_{3}, \neg A \odot \neg A
\end{array}
$$

Figure 10. $\tau_{\left\{H_{1}^{c}: A \Rightarrow p_{2}, H_{2}^{c}: A \Rightarrow p_{4}\right\}}^{*}$ and $\tau_{\left\{H_{1}^{c}: A \Rightarrow p_{5}, H_{2}^{c}: A \Rightarrow p_{3}\right\}}^{*}$

$$
\begin{gathered}
\frac{C \Rightarrow C \quad p_{1}, p_{2} \Rightarrow A \odot A}{p_{1} \Rightarrow C \mid C, p_{2} \Rightarrow A \odot A} \quad \frac{C \Rightarrow C \quad p_{3}, p_{4} \Rightarrow A \odot A}{p_{3} \Rightarrow C \mid C, p_{4} \Rightarrow A \odot A} \\
\frac{C \Rightarrow C \quad p_{5}, p_{6} \Rightarrow A \odot A}{p_{5} \Rightarrow C \mid C, p_{6} \Rightarrow A \odot A}
\end{gathered}
$$

Figure 11. $\tau_{H_{3}^{c}: p_{1}, p_{2} \Rightarrow A \odot A}^{*} \tau_{H_{3}^{c}: p_{3}, p_{4} \Rightarrow A \odot A}^{*}$ and $\tau_{H_{3}^{c}: p_{5}, p_{6} \Rightarrow A \odot A}^{*}$

Step 3 (separation of one branch). A proof $\tau_{H_{1}^{c}: G \mid G^{*}}^{\xi(2)}$ is constructed by applying sequentially

$$
\tau_{H_{3}^{c}: p_{3}, p_{4} \Rightarrow A \odot A}, \tau_{H_{3}^{c}: p_{5}, p_{6} \Rightarrow A \odot A}^{*}
$$

to $p_{3}, p_{4} \Rightarrow A \odot A$ and $p_{5}, p_{6} \Rightarrow A \odot A$ in $G_{H_{1}^{c}: G \mid G^{*}}^{\text {is }(1)}$ as shown in Figure 12 , where $G^{\prime \prime} \equiv$ $G_{H_{1}^{c}: G \mid G^{*}}^{i(1)} \backslash\left\{p_{3}, p_{4} \Rightarrow A \odot A, p_{5}, p_{6} \Rightarrow A \odot A\right\}$

$$
\begin{gathered}
G_{H_{1}^{c}: G \mid G^{*}}^{\mathfrak{i n}(2)}=\Rightarrow p_{2}, B\left|B \Rightarrow p_{4}, \neg A \odot \neg A\right| p_{1} \Rightarrow C\left|C, p_{2} \Rightarrow A \odot A\right| A \Rightarrow p_{3}\left|\Rightarrow p_{1}, B\right| \\
B \Rightarrow p_{6}, \neg A \odot \neg A\left|A \Rightarrow p_{5}\right| p_{3} \Rightarrow C\left|C, p_{4} \Rightarrow A \odot A\right| p_{5} \Rightarrow C \mid C, p_{6} \Rightarrow A \odot A .
\end{gathered}
$$

$$
\begin{gathered}
C \Rightarrow C \frac{C \Rightarrow C G^{\prime \prime}\left|p_{3}, p_{4} \Rightarrow A \odot A\right| p_{5}, p_{6} \Rightarrow A \odot A}{G^{\prime \prime}\left|p_{3} \Rightarrow C\right| C, p_{4} \Rightarrow A \odot A \mid p_{5}, p_{6} \Rightarrow A \odot A} \\
\frac{G_{H_{1}^{c}: G \mid G^{*}}^{\tilde{h}(2)}}{G^{\prime \prime}}\left|{p_{3}}^{\prime} \Rightarrow C\right| C, p_{4} \Rightarrow A \odot A\left|p_{5} \Rightarrow C\right| C, p_{6} \Rightarrow A \odot A
\end{gathered}
$$

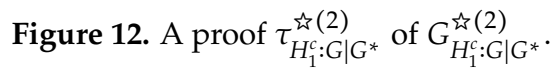

Notice that

$$
\begin{aligned}
& \mathcal{D}\left(B \Rightarrow p_{4}, \neg A \odot \neg A\left|A \Rightarrow p_{3}\right| p_{3} \Rightarrow C \mid C, p_{4} \Rightarrow A \odot A\right) \\
= & \mathcal{D}\left(B \Rightarrow p_{6}, \neg A \odot \neg A\left|A \Rightarrow p_{5}\right| p_{5} \Rightarrow C \mid C, p_{6} \Rightarrow A \odot A\right) \\
= & A \Rightarrow C \mid C, B \Rightarrow A \odot A, \neg A \odot \neg A .
\end{aligned}
$$

Then it is permissible to cut off the part

$$
B \Rightarrow p_{6}, \neg A \odot \neg A\left|A \Rightarrow p_{5}\right| p_{5} \Rightarrow C \mid C, p_{6} \Rightarrow A \odot A
$$

of $G_{H_{1}^{c}: G \mid G^{*}}^{i(2)}$, which corresponds to applying $(E C)$ to $\mathcal{D}\left(G_{H_{1}^{c}: G \mid G^{*}}^{i(2)}\right)$. We regard such a manipulation as

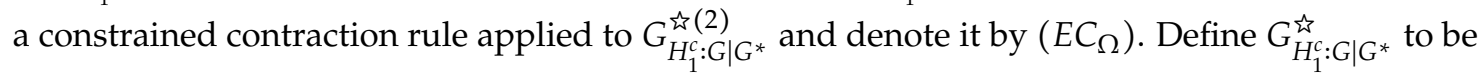

$$
\Rightarrow p_{2}, B\left|B \Rightarrow p_{4}, \neg A \odot \neg A\right| p_{1} \Rightarrow C\left|C, p_{2} \Rightarrow A \odot A\right|
$$




$$
A \Rightarrow p_{3}\left|\Rightarrow p_{1}, B\right| p_{3} \Rightarrow C \mid C, p_{4} \Rightarrow A \odot A .
$$

Then we construct a proof of $G_{H_{1}^{c}: G \mid G^{*}}^{i ⿱ 乛}$ by $\frac{G_{H_{1}^{c}: G \mid G^{*}}^{i c(2)}}{G_{H_{1}^{c}: G \mid G^{*}}^{i j}}\left(E C_{\Omega}\right)$, which guarantees the validity of

$$
\vdash_{\text {GIUL }} \mathcal{D}\left(G_{H_{1}^{c}: G \mid G^{*}}^{i \zeta}\right)
$$

under the condition

$$
\vdash_{\text {GIUL }} \mathcal{D}\left(G_{H_{1}^{c}: G \mid G^{*}}^{i(2)}\right) .
$$

A change happens here! There is only one sequent which is a copy of a sequent in $G^{*}$ in $G_{H_{1}^{c}: G \mid G^{*}}^{i}$. It is simpler than $G \mid G^{*}$. So we are moving forward. The above procedure is called the separation of $G \mid G^{*}$ as a branch of $H_{1}^{c}$ and reformulated as follows (See Section 7 for details).

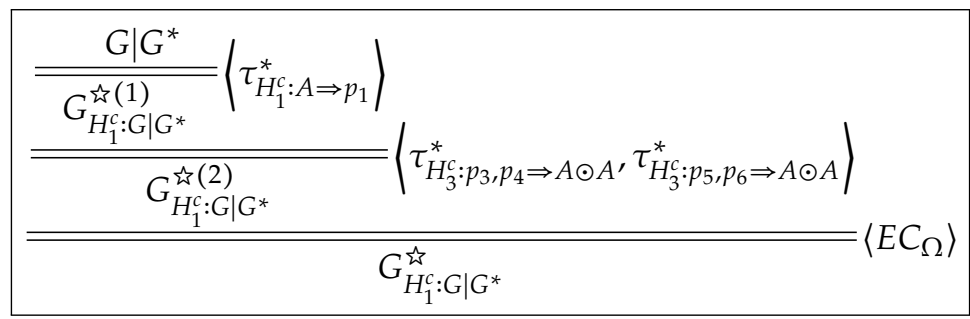

The separation of $G \mid G^{*}$ as a branch of $H_{2}^{c}$ is constructed by a similar procedure as follows.

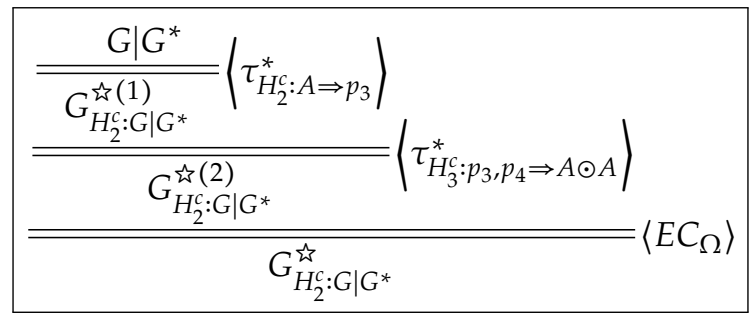

Note that $\mathcal{D}\left(G_{H_{1}^{c}: G \mid G^{*}}^{\grave{3}}\right)=H_{2}$ and $\mathcal{D}\left(G_{H_{2}^{c}: G \mid G^{*}}^{\dot{\xi}}\right)=H_{3}$. So we have built up one-one correspondences between proofs of $G_{H_{1}^{c}: G \mid G^{*}}^{i 3}, G_{H_{1}^{c}: G \mid G^{*}}^{i s}$ and those of $H_{2}, H_{3}$.

Step 3 (separation algorithm of multiple branches). We will prove $\vdash_{\text {GIUL }} \mathcal{D}_{0}\left(G_{0}\right)$ in a direct way, i.e., only the major step of Theorem 2 is presented in the following. (See Appendix A.5.4 for a complete illustration.) Recall that

$$
\begin{aligned}
& G_{H_{1}^{c}: G \mid G^{*}}^{\check{\imath}^{*}}=\Rightarrow p_{2}, B\left|B \Rightarrow p_{4}, \neg A \odot \neg A\right| p_{1} \Rightarrow C\left|C, p_{2} \Rightarrow A \odot A\right| \\
& A \Rightarrow p_{3}\left|\Rightarrow p_{1}, B\right| p_{3} \Rightarrow C \mid C, p_{4} \Rightarrow A \odot A, \\
& G_{H_{2}^{c}: G \mid G^{*}}^{i s}=A \Rightarrow p_{1}\left|\Rightarrow p_{2}, B\right| B \Rightarrow p_{4}, \neg A \odot \neg A\left|p_{1} \Rightarrow C\right| C, p_{2} \Rightarrow A \odot A \mid \\
& B \Rightarrow p_{3}, \neg A \odot \neg A\left|p_{3} \Rightarrow C\right| C, p_{4} \Rightarrow A \odot A .
\end{aligned}
$$

By reassigning identification numbers to occurrences of $p^{\prime} s$ in $G_{H_{2}^{c}: G \mid G^{*}}^{i d}$

$$
\begin{gathered}
G_{H_{2}^{c}: G \mid G^{*}}^{\grave{\hbar}}=A \Rightarrow p_{5}\left|\Rightarrow p_{6}, B\right| B \Rightarrow p_{8}, \neg A \odot \neg A\left|p_{5} \Rightarrow C\right| C, p_{6} \Rightarrow A \odot A \mid \\
B \Rightarrow p_{7}, \neg A \odot \neg A\left|p_{7} \Rightarrow C\right| C, p_{8} \Rightarrow A \odot A .
\end{gathered}
$$


By applying $\tau_{\left\{H_{1}^{c}: A \Rightarrow p_{5}, H_{2}^{c}: A \Rightarrow p_{3}\right\}}^{*}$ to $A \Rightarrow p_{3}$ in $G_{H_{1}^{c}: G \mid G^{*}}^{\tilde{\imath}}$ and $A \Rightarrow p_{5}$ in $G_{H_{2}^{c}: G \mid G^{*}}^{\hat{i}}$, we get $\vdash_{\text {GIUL }}$ $G^{\prime}$, where

$$
\begin{gathered}
G^{\prime} \equiv \Rightarrow p_{2}, B\left|B \Rightarrow p_{4}, \neg A \odot \neg A\right| p_{1} \Rightarrow C\left|C, p_{2} \Rightarrow A \odot A\right| \Rightarrow p_{1}, B \mid \\
p_{3} \Rightarrow C\left|C, p_{4} \Rightarrow A \odot A\right| \Rightarrow p_{6}, B\left|B \Rightarrow p_{8}, \neg A \odot \neg A\right| p_{5} \Rightarrow C\left|C, p_{6} \Rightarrow A \odot A\right| \\
B \Rightarrow p_{7}, \neg A \odot \neg A\left|p_{7} \Rightarrow C\right| C, p_{8} \Rightarrow A \odot A\left|\Rightarrow p_{5}, B\right| B \Rightarrow p_{3}, \neg A \odot \neg A .
\end{gathered}
$$

Why reassign identification numbers to occurrences of $p^{\prime} s$ in $G_{H_{2}^{c}: G \mid G^{*}}^{\text {i }}$ ? It makes different occurrences of $p^{\prime} s$ to be assigned different identification numbers in two nodes $G_{H_{1}^{c}: G \mid G^{*}}^{i j}$ and $G_{H_{2}^{c}: G \mid G^{*}}^{i j}$ of the proof of $G^{\prime}$.

By applying $\left\langle E C_{\Omega}^{*}\right\rangle$ to $G^{\prime}$, we get $\vdash_{\mathrm{GIUL}_{\Omega}} G_{\mathrm{I}}^{\text {is }}$, where

$$
\begin{gathered}
G_{\mathbf{I}}^{\text {¿ }} \equiv \Rightarrow p_{2}, B\left|B \Rightarrow p_{4}, \neg A \odot \neg A\right| p_{1} \Rightarrow C\left|C, p_{2} \Rightarrow A \odot A\right| \Rightarrow p_{1}, B \mid \\
p_{3} \Rightarrow C\left|C, p_{4} \Rightarrow A \odot A\right| B \Rightarrow p_{3}, \neg A \odot \neg A .
\end{gathered}
$$

A great change happens here! We have eliminated all sequents which are copies of some sequents in $G^{*}$ and converted $G \mid G^{*}$ into $G_{I}^{\text {is }}$ in which each sequent is some copy of a sequent in $G_{0}$.

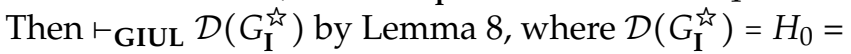

$$
\Rightarrow C, B|C \Rightarrow B, A \odot A| B \Rightarrow C, \neg A \odot \neg A \mid C, B \Rightarrow A \odot A, \neg A \odot \neg A .
$$

\begin{tabular}{|c|c|}
\hline \multicolumn{2}{|c|}{$\begin{array}{l}G\left|G^{*}=\Rightarrow p_{2}, B\right| B \Rightarrow p_{4}, \neg A \odot \neg A\left|p_{1} \Rightarrow C\right| \\
C, p_{2} \Rightarrow A \odot A\left|A \Rightarrow p_{1}\right| A \Rightarrow p_{3} \mid p_{3}, p_{4} \Rightarrow A \odot A\end{array}$} \\
\hline$\because \vdots \bullet^{\circ}$ & $\because \because{ }^{\circ}$ \\
\hline $\begin{array}{l}G_{H_{2}^{c}: G \mid G^{*}}^{\varkappa^{*}}=A \Rightarrow p_{1}\left|\Rightarrow p_{2}, B\right| \\
B \Rightarrow p_{4}, \neg A \odot \neg A\left|p_{1} \Rightarrow C\right| \\
C, p_{2} \Rightarrow A \odot A\left|p_{3} \Rightarrow C\right| \\
B \Rightarrow p_{3}, \neg A \odot \neg A \mid C, p_{4} \Rightarrow A \odot A\end{array}$ & $\begin{array}{l}G_{H_{1}^{c}: G \mid G^{*}}^{i} \Rightarrow p_{2}, B\left|B \Rightarrow p_{4}, \neg A \odot \neg A\right| \\
p_{1} \Rightarrow C\left|C, p_{2} \Rightarrow A \odot A\right| A \Rightarrow p_{3} \mid \\
A \Rightarrow p_{1}, B\left|p_{3} \Rightarrow C\right| C, p_{4} \Rightarrow A \odot A\end{array}$ \\
\hline \multicolumn{2}{|r|}{$\because \vdots \bullet$} \\
\hline \multicolumn{2}{|c|}{$\begin{array}{l}G_{\mathrm{I}}^{\tilde{I}^{2}}=p_{2}, B\left|B \Rightarrow p_{4}, \neg A \odot \neg A\right| p_{1} \Rightarrow C\left|C, p_{2} \Rightarrow A \odot A\right| \\
B \Rightarrow \neg A \odot \neg A, p_{3}\left|\Rightarrow p_{1}, B\right| p_{3} \Rightarrow C \mid C, p_{4} \Rightarrow A \odot A\end{array}$} \\
\hline
\end{tabular}

So we have built up one-one correspondences between the proof of $G_{I}^{\text {i }}$ and that of $H_{0}$, i.e., the proof of $H_{0}$ can be constructed by applying $(\mathcal{D})$ to the proof of $G_{I}^{\text {i }}$. The major steps of constructing

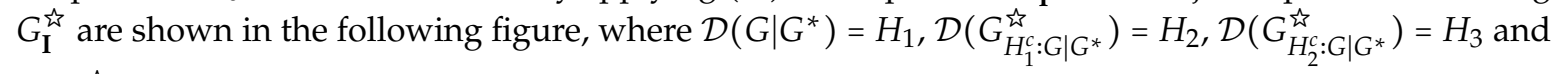
$\mathcal{D}\left(G_{\mathbf{I}}^{\text {¿ }}\right)=H_{0}$.

In the above example, $\mathcal{D}\left(G_{\mathbf{I}}^{\hat{i}}\right)=\mathcal{D}_{0}\left(G_{0}\right)$. But that is not always the case. In general, we can prove that $\vdash_{\mathrm{GL}} \mathcal{D}_{0}\left(G_{0}\right)$ if $\vdash_{\mathbf{G L}} \mathcal{D}\left(G_{\mathbf{I}}^{\mathfrak{i}}\right)$, which is shown in the proof of the main theorem in Page 42 . This example shows that the proof of the main theorem essentially presents an algorithm to construct a proof of $\mathcal{D}_{0}\left(G_{0}\right)$ from $\tau$.

\section{Preprocessing of Proof Tree}

Let $\tau$ be a cut-free proof of $G_{0}$ in the main theorem in GL by Lemma 1 . Starting with $\tau$, we will construct a proof $\tau^{*}$ which contains no application of $(E C)$ and has some other properties in this section. 
Lemma 2. (i) If $\vdash_{\mathrm{GL}} \Gamma_{1} \Rightarrow A, \Delta_{1}$ and $\vdash_{\mathrm{GL}} \Gamma_{2} \Rightarrow B, \Delta_{2}$

$$
\text { then } \vdash_{\mathrm{GL}} \Gamma_{1} \Rightarrow A \wedge B, \Delta_{1} \mid \Gamma_{2} \Rightarrow A \wedge B, \Delta_{2} ;
$$

(ii) If $\vdash_{\mathrm{GL}} \Gamma_{1}, A \Rightarrow \Delta_{1}$ and $\vdash_{\mathrm{GL}} \Gamma_{2}, B \Rightarrow \Delta_{2}$

$$
\text { then } \vdash_{\mathrm{GL}} \Gamma_{1}, A \vee B \Rightarrow \Delta_{1} \mid \Gamma_{2}, A \vee B \Rightarrow \Delta_{2} .
$$

Proof. (i)

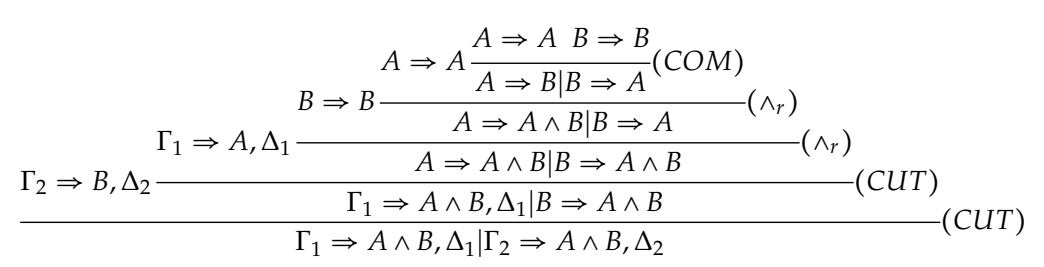

(ii) is proved by a procedure similar to that of (i) and omitted.

We introduce two new rules by Lemma 2 .

Definition 12. $\frac{G_{1}\left|\Gamma_{1} \Rightarrow A, \Delta_{1} \quad G_{2}\right| \Gamma_{2} \Rightarrow B, \Delta_{2}}{G_{1}\left|G_{2}\right| \Gamma_{1} \Rightarrow A \wedge B, \Delta_{1} \mid \Gamma_{2} \Rightarrow A \wedge B, \Delta_{2}}(\wedge$ rw $)$ and $\frac{G_{1}\left|\Gamma_{1}, A \Rightarrow \Delta_{1} \quad G_{2}\right| \Gamma_{2}, B \Rightarrow \Delta_{2}}{G_{1}\left|G_{2}\right| \Gamma_{1}, A \vee B \Rightarrow \Delta_{1} \mid \Gamma_{2}, A \vee B \Rightarrow \Delta_{2}}\left(\vee_{l w}\right)$ are called the generalized $\left(\wedge_{r}\right)$ and $\left(\vee_{l}\right)$ rules, respectively.

Now, we begin to process $\tau$ as follows.

Step 1. A proof $\tau^{1}$ is constructed by replacing inductively all applications of

$$
\frac{G_{1}\left|\Gamma \Rightarrow A, \Delta \quad G_{2}\right| \Gamma \Rightarrow B, \Delta}{G_{1}\left|G_{2}\right| \Gamma \Rightarrow A \wedge B, \Delta}\left(\wedge_{r}\right) \quad\left(\text { or } \frac{G_{1}\left|\Gamma, A \Rightarrow \Delta \quad G_{2}\right| \Gamma, B \Rightarrow \Delta}{G_{1}\left|G_{2}\right| \Gamma, A \vee B \Rightarrow \Delta}\left(\vee_{l}\right)\right)
$$

in $\tau$ with

$$
\begin{gathered}
\frac{G_{1}\left|\Gamma \Rightarrow A, \Delta \quad G_{2}\right| \Gamma \Rightarrow B, \Delta}{\frac{G_{1}\left|G_{2}\right| \Gamma \Rightarrow A \wedge B, \Delta \mid \Gamma \Rightarrow A \wedge B, \Delta}{G_{1}\left|G_{2}\right| \Gamma \Rightarrow A \wedge B, \Delta}(\wedge r w)}(E C) \\
\text { (accordingly } \left.\frac{G_{1}\left|\Gamma, A \Rightarrow \Delta \quad G_{2}\right| \Gamma, B \Rightarrow \Delta}{\frac{G_{1}\left|G_{2}\right| \Gamma, A \vee B \Rightarrow \Delta \mid \Gamma, A \vee B \Rightarrow \Delta}{G_{1}\left|G_{2}\right| \Gamma, A \vee B \Rightarrow \Delta}\left(\vee_{l w}\right)}(E C) \text { for }\left(\vee_{l}\right)\right) .
\end{gathered}
$$

The replacements in Step 1 are local and the root of $\tau^{1}$ is also labeled by $G_{0}$.

Definition 13. We sometimes may regard $\frac{G^{\prime}}{G^{\prime}}$ as a structural rule of $\mathbf{G} \mathbf{L}$ and denote it by $\left(I D_{\Omega}\right)$ for convenience. The focus sequent for $\left(I D_{\Omega}\right)$ is undefined.

Lemma 3. Let $\frac{G^{\prime} \mid S^{m}}{G^{\prime} \mid S}\left(E C^{*}\right) \in \tau^{1}, T h_{\tau^{1}}\left(G^{\prime} \mid S\right)=\left(H_{0}, H_{1}, \cdots, H_{n}\right)$, where $H_{0}=G^{\prime} \mid S$ and $H_{n}=G_{0}$. A tree $\tau^{\prime}$ is constructed by replacing each $H_{k}$ in $\tau^{1}$ with $H_{k} \mid S^{m-1}$ for all $0 \leqslant k \leqslant n$. Then $\tau^{\prime}$ is a proof of $G_{0} \mid S^{m-1}$.

Proof. The proof is by induction on $n$. Since $\tau^{1}\left(G^{\prime} \mid S^{m}\right)$ is a proof and $\frac{G^{\prime} \mid S^{m}}{H_{0} \mid S^{m-1}}\left(I D_{\Omega}\right)$ is valid in $\mathbf{G} \mathbf{L}$, then $\tau^{\prime}\left(H_{0} \mid S^{m-1}\right)$ is a proof. Suppose that $\tau^{\prime}\left(H_{n-1} \mid S^{m-1}\right)$ is a proof. Since $\frac{H_{n-1} G^{\prime \prime}}{H_{n}}(I I)$ 
(or $\left.\frac{H_{n-1}}{H_{n}}(I)\right)$ in $\tau^{1}$, then $\frac{H_{n-1} \mid S^{m-1} \quad G^{\prime \prime}}{H_{n} \mid S^{m-1}}$ (or $\left.\frac{H_{n-1} \mid S^{m-1}}{H_{n} \mid S^{m-1}}\right)$ is an application of the same rule (II) (or $(I)$ ). Thus $\tau^{\prime}\left(H_{n} \mid S^{m-1}\right)$ is a proof.

Definition 14. The manipulation described in Lemma 3 is called a sequent-inserting operation.

Clearly, the number of (EC*)-applications in $\tau^{\prime}$ is less than $\tau^{1}$. Next, we continue to process $\tau$. Step 2. Let $\frac{G_{1}^{\prime \prime \prime} \mid\left\{S_{1}^{c}\right\}^{m_{1}^{\prime}}}{G_{1}^{\prime} \mid S_{1}^{c}}\left(E C^{*}\right), \cdots, \frac{G_{N}^{\prime \prime \prime} \mid\left\{S_{N}^{c}\right\}^{m_{N}^{\prime}}}{G_{N}^{\prime} \mid S_{N}^{c}}\left(E C^{*}\right)$ be all applications of $\left(E C^{*}\right)$ in $\tau^{1}$ and $G_{0}^{*}:=$ $\left\{S_{1}^{c}\right\}^{m_{1}^{\prime}-1}|\cdots|\left\{S_{N}^{c}\right\}^{m_{N}^{\prime}-1}$. By repeatedly applying sequent-inserting operations, we construct a proof of $G_{0} \mid G_{0}^{*}$ in $\mathbf{G L}$ without applications of $\left(E C^{*}\right)$ and denote it by $\tau^{2}$.

Remark 1. (i) $\tau^{2}$ is constructed by converting (EC) into $\left(I D_{\Omega}\right)$; (ii) Each node of $\tau^{2}$ has the form $H_{0} \mid H_{0}^{*}$, where $H_{0} \in \tau^{1}$ and $H_{0}^{*}$ is a (possibly empty) subset of $G_{0}^{*}$.

We need the following construction to eliminate applications of $(E W)$ in $\tau^{2}$.

Construction 1. Let $H \in \tau^{2}, H^{\prime} \subseteq H$ and $T h_{\tau^{2}}(H)=\left(H_{0}, \cdots, H_{n}\right)$, where $H_{0}=H, H_{n}=G_{0} \mid G_{0}^{*}$. Hypersequents $\left\langle H_{k}\right\rangle_{H: H^{\prime}}$ and trees $\tau_{H: H^{\prime}}^{2}\left(\left\langle H_{k}\right\rangle_{H: H^{\prime}}\right)$ for all $0 \leqslant k \leqslant n$ are constructed inductively as follows.

(i) $\left\langle H_{0}\right\rangle_{H: H^{\prime}}:=H^{\prime}$ and $\tau_{H: H^{\prime}}^{2}\left(\left\langle H_{0}\right\rangle_{H: H^{\prime}}\right)$ consists of a single node $H^{\prime}$;

(ii) Let $\frac{G^{\prime}\left|S^{\prime} \quad G^{\prime \prime}\right| S^{\prime \prime}}{G^{\prime}\left|G^{\prime \prime}\right| H^{\prime \prime}}(I I)$ (or $\frac{G^{\prime} \mid S^{\prime}}{G^{\prime} \mid S^{\prime \prime}}(I)$ ) be in $\tau^{2}, H_{k}=G^{\prime} \mid S^{\prime}$ and $H_{k+1}=G^{\prime}\left|G^{\prime \prime}\right| H^{\prime \prime}$ (accordingly $H_{k+1}=G^{\prime} \mid S^{\prime \prime}$ for $\left.(I)\right)$ for some $0 \leqslant k \leqslant n-1$.

If $S^{\prime} \in\left\langle H_{k}\right\rangle_{H: H^{\prime}}$

$$
\begin{gathered}
\left\langle H_{k+1}\right\rangle_{H: H^{\prime}}:=\left\langle H_{k}\right\rangle_{H: H^{\prime}} \backslash\left\{S^{\prime}\right\}\left|G^{\prime \prime}\right| H^{\prime \prime} \\
\text { (accordingly }\left\langle H_{k+1}\right\rangle_{H: H^{\prime}}:=\left\langle H_{k}\right\rangle_{H: H^{\prime}} \backslash\left\{S^{\prime}\right\} \mid S^{\prime \prime} \text { for }(I) \text { ) }
\end{gathered}
$$

and $\tau_{H: H^{\prime}}^{2}\left(\left\langle H_{k+1}\right\rangle_{H: H^{\prime}}\right)$ is constructed by combining trees

$$
\begin{gathered}
\tau_{H: H^{\prime}}^{2}\left(\left\langle H_{k}\right\rangle_{H: H^{\prime}}\right), \tau^{2}\left(G^{\prime \prime} \mid S^{\prime \prime}\right) \text { with } \frac{\left\langle H_{k}\right\rangle_{H: H^{\prime}} G^{\prime \prime} \mid S^{\prime \prime}}{\left\langle H_{k+1}\right\rangle_{H: H^{\prime}}}(I I) \\
\text { (accordingly } \left.\tau_{H: H^{\prime}}^{2}\left(\left\langle H_{k}\right\rangle_{H: H^{\prime}}\right) \text { with } \frac{\left\langle H_{k}\right\rangle_{H: H^{\prime}}}{\left\langle H_{k+1}\right\rangle_{H: H^{\prime}}}(I) \text { for }(I)\right)
\end{gathered}
$$

otherwise $\left\langle H_{k+1}\right\rangle_{H: H^{\prime}}:=\left\langle H_{k}\right\rangle_{H: H^{\prime}}$ and $\tau_{H: H^{\prime}}^{2}\left(\left\langle H_{k+1}\right\rangle_{H: H^{\prime}}\right)$ is constructed by combining

$$
\tau_{H: H^{\prime}}^{2}\left(\left\langle H_{k}\right\rangle_{H: H^{\prime}}\right) \text { with } \frac{\left\langle H_{k}\right\rangle_{H: H^{\prime}}}{\left\langle H_{k+1}\right\rangle_{H: H^{\prime}}}\left(I D_{\Omega}\right) .
$$

(iii) Let $\frac{G^{\prime}}{G^{\prime} \mid S^{\prime}}(E W) \in \tau^{2}, H_{k}=G^{\prime}$ and $H_{k+1}=G^{\prime} \mid S^{\prime}$ then $\left\langle H_{k+1}\right\rangle_{H: H^{\prime}}:=\left\langle H_{k}\right\rangle_{H: H^{\prime}}$ and $\tau_{H: H^{\prime}}^{2}\left(\left\langle H_{k+1}\right\rangle_{H: H^{\prime}}\right)$ is constructed by combining $\tau_{H: H^{\prime}}^{2}\left(\left\langle H_{k}\right\rangle_{H: H^{\prime}}\right)$ with $\frac{\left\langle H_{k}\right\rangle_{H: H^{\prime}}}{\left\langle H_{k+1}\right\rangle_{H: H^{\prime}}}\left(I D_{\Omega}\right)$.

Lemma 4. (i) $\left\langle H_{k}\right\rangle_{H: H^{\prime}} \subseteq H_{k}$ for all $0 \leqslant k \leqslant n$;

(ii) $\tau_{H: H^{\prime}}^{2}\left(\left\langle H_{k}\right\rangle_{H: H^{\prime}}\right)$ is a derivation of $\left\langle H_{k}\right\rangle_{H: H^{\prime}}$ from $H^{\prime}$ without $(E C)$.

Proof. The proof is by induction on $k$. For the base step, $\left\langle H_{0}\right\rangle_{H: H^{\prime}}=H^{\prime}$ and $\tau_{H: H^{\prime}}^{2}\left(\left\langle H_{0}\right\rangle_{H: H^{\prime}}\right)$ consists of a single node $H^{\prime}$. Then $\left\langle H_{0}\right\rangle_{H: H^{\prime}} \subseteq H_{0}=H, \tau_{H: H^{\prime}}^{2}\left(\left\langle H_{0}\right\rangle_{H: H^{\prime}}\right)$ is a derivation of $\left\langle H_{0}\right\rangle_{H: H^{\prime}}$ from $H^{\prime}$ without $(E C)$. 
For the induction step, suppose that $\left\langle H_{k}\right\rangle_{H: H^{\prime}}$ and $\tau_{H: H^{\prime}}^{2}\left(\left\langle H_{k}\right\rangle_{H: H^{\prime}}\right)$ be constructed such that (i) and (ii) hold for some $0 \leqslant k \leqslant n-1$. There are two cases to be considered.

Case 1. Let $\frac{G^{\prime} \mid S^{\prime}}{G^{\prime} \mid S^{\prime \prime}}(I) \in \tau^{2}, H_{k}=G^{\prime} \mid S^{\prime}$ and $H_{k+1}=G^{\prime} \mid S^{\prime \prime}$. If $S^{\prime} \in\left\langle H_{k}\right\rangle_{H: H^{\prime}}$ then $\left\langle H_{k}\right\rangle_{H: H^{\prime}} \backslash\left\{S^{\prime}\right\} \subseteq G^{\prime}$ by $\left\langle H_{k}\right\rangle_{H: H^{\prime}} \subseteq H_{k}=G^{\prime} \mid S^{\prime}$. Thus $\left\langle H_{k+1}\right\rangle_{H: H^{\prime}}=\left(\left\langle H_{k}\right\rangle_{H: H^{\prime}} \backslash\left\{S^{\prime}\right\}\right)\left|S^{\prime \prime} \subseteq G^{\prime}\right| S^{\prime \prime}=H_{k+1}$. Otherwise $S^{\prime} \notin\left\langle H_{k}\right\rangle_{H: H^{\prime}}$ then $\left\langle H_{k}\right\rangle_{H: H^{\prime}} \subseteq G^{\prime}$ by $\left\langle H_{k}\right\rangle_{H: H^{\prime}} \subseteq H_{k}=G^{\prime} \mid S^{\prime}$. Thus $\left\langle H_{k+1}\right\rangle_{H: H^{\prime}} \subseteq H_{k+1}$ by $\left\langle H_{k+1}\right\rangle_{H: H^{\prime}}=\left\langle H_{k}\right\rangle_{H: H^{\prime}} \subseteq G^{\prime} \subseteq H_{k+1} \cdot \tau_{H: H^{\prime}}^{2}\left(\left\langle H_{k+1}\right\rangle_{H: H^{\prime}}\right)$ is a derivation of $\left\langle H_{k+1}\right\rangle_{H: H^{\prime}}$ from $H^{\prime}$ without (EC) since $\tau_{H: H^{\prime}}^{2}\left(\left\langle H_{k}\right\rangle_{H: H^{\prime}}\right)$ is such one and $\frac{\left\langle H_{k}\right\rangle_{H: H^{\prime}}}{\left\langle H_{k+1}\right\rangle_{H: H^{\prime}}}(I)$ is a valid instance of a rule (I) of GL. The case of applications of the two-premise rule is proved by a similar procedure and omitted.

Case 2. Let $\frac{G^{\prime}}{G^{\prime} \mid S^{\prime}}(E W) \in \tau^{2}, H_{k}=G^{\prime}$ and $H_{k+1}=G^{\prime} \mid S^{\prime}$. Then $\left\langle H_{k+1}\right\rangle_{H: H^{\prime}} \subseteq H_{k+1}$ by $\left\langle H_{k+1}\right\rangle_{H: H^{\prime}}=$ $\left\langle H_{k}\right\rangle_{H: H^{\prime}} \subseteq H_{k} \subseteq H_{k+1} \cdot \tau_{H: H^{\prime}}^{2}\left(\left\langle H_{k+1}\right\rangle_{H: H^{\prime}}\right)$ is a derivation of $\left\langle H_{k+1}\right\rangle_{H: H^{\prime}}$ from $H^{\prime}$ without $(E C)$ since $\tau_{H: H^{\prime}}^{2}\left(\left\langle H_{k}\right\rangle_{H: H^{\prime}}\right)$ is such one and $\frac{\left\langle H_{k}\right\rangle_{H: H^{\prime}}}{\left\langle H_{k+1}\right\rangle_{H: H^{\prime}}}\left(I D_{\Omega}\right)$ is valid by Definition 13.

Definition 15. The manipulation described in Construction 1 is called a derivation-pruning operation.

Notation 3. We denote $\left\langle H_{n}\right\rangle_{H: H^{\prime}}$ by $G_{H: H^{\prime}}^{2} \tau_{H: H^{\prime}}^{2}\left(\left\langle H_{n}\right\rangle_{H: H^{\prime}}\right)$ by $\tau_{H: H^{\prime}}^{2}$ and say that $H^{\prime}$ is transformed into $G_{H: H^{\prime}}^{2}$ in $\tau^{2}$

Then Lemma 4 shows that $\frac{H^{\prime}}{G_{H: H^{\prime}}^{2}}\left\langle\tau_{H: H^{\prime}}^{2}\right\rangle, G_{H: H^{\prime}}^{2} \subseteq G_{0} \mid G_{0}^{*}$. Now, we continue to process $\tau$ as follows.

Step 3. Let $\frac{G^{\prime}}{G^{\prime} \mid S^{\prime}}(E W) \in \tau^{2}$ then $\tau_{G^{\prime} \mid S^{\prime}: G^{\prime}}^{2}\left(\left\langle H_{n}\right\rangle_{G^{\prime} \mid S^{\prime}: G^{\prime}}\right)$ is a derivation of $\left\langle H_{n}\right\rangle_{G^{\prime} \mid S^{\prime}: G^{\prime}}$ from $G^{\prime}$ thus a proof of $\left\langle H_{n}\right\rangle_{G^{\prime} \mid S^{\prime}: G^{\prime}}$ is constructed by combining $\tau^{2}\left(G^{\prime}\right)$ and $\tau_{G^{\prime} \mid S^{\prime}: G^{\prime}}^{2}\left(\left\langle H_{n}\right\rangle_{G^{\prime} \mid S^{\prime}: G^{\prime}}\right)$ with $\frac{G^{\prime}}{G^{\prime}}\left(I D_{\Omega}\right)$. By repeatedly applying the procedure above, we construct a proof $\tau^{3}$ of $G_{1} \mid G_{1}^{*}$ without $(E W)$ in $\mathbf{G L}$, where $G_{1} \subseteq G_{0}, G_{1}^{*} \subseteq G_{0}^{*}$ by Lemma $4(i)$.

Step 4. Let $\Gamma, p, \perp \Rightarrow \Delta \in \tau^{3}$ (or $\left.\Gamma, p \Rightarrow \mathrm{T}, \Delta, \frac{G \mid \Gamma \Rightarrow \Delta}{G \mid \Gamma, p \Rightarrow \Delta}(W L)\right)$ then there exists $\Gamma^{\prime} \Rightarrow \Delta^{\prime} \in H$ such that $p \in \Gamma^{\prime}$ for all $H \in T h_{\tau^{3}}(\Gamma, p, \perp \Rightarrow \Delta)$ (accordingly $H \in T h_{\tau^{3}}(\Gamma, p \Rightarrow T, \Delta), H \in T h_{\tau^{3}}(\Gamma, p \Rightarrow \Delta)$ ) thus a proof is constructed by replacing top-down $p$ in each $\Gamma^{\prime}$ with $T$.

Let $\Gamma, \perp \Rightarrow p, \Delta\left(\right.$ or $\left.\Gamma \Rightarrow T, p, \Delta, \frac{G \mid \Gamma \Rightarrow \Delta}{G \mid \Gamma \Rightarrow p, \Delta}(W R)\right)$ is a leaf of $\tau^{3}$ then there exists $\Gamma^{\prime} \Rightarrow \Delta^{\prime} \in H$ such that $p \in \Delta^{\prime}$ for all $H \in T h_{\tau^{3}}(\Gamma, \perp \Rightarrow p, \Delta)$ (accordingly $H \in T h_{\tau^{3}}(\Gamma \Rightarrow T, p, \Delta)$ or $H \in T h_{\tau^{3}}(\Gamma \Rightarrow p, \Delta)$ ) thus a proof is constructed by replacing top-down $p$ in each $\Gamma^{\prime}$ with $\perp$.

Repeatedly applying the procedure above, we construct a proof $\tau^{4}$ of $G_{2} \mid G_{2}^{*}$ in GL such that there does not exist occurrence of $p$ in $\Gamma$ or $\Delta$ at each leaf labeled by $\Gamma, \perp \Rightarrow \Delta$ or $\Gamma \Rightarrow T, \Delta$, or $p$ is not the weakening formula $A$ in $\frac{G \mid \Gamma \Rightarrow \Delta}{G \mid \Gamma \Rightarrow A, \Delta}(W R)$ or $\frac{G \mid \Gamma \Rightarrow \Delta}{G \mid \Gamma, A \Rightarrow \Delta}(W L)$ when $(W R)$ or $(W L)$ is available. Define two operations $\sigma_{l}$ and $\sigma_{r}$ on sequents by $\sigma_{l}(\Gamma, p \Rightarrow \Delta):=\Gamma, \top \Rightarrow \Delta$ and $\sigma_{r}(\Gamma \Rightarrow p, \Delta):=\Gamma \Rightarrow \perp, \Delta$. Then $G_{2} \mid G_{2}^{*}$ is obtained by applying $\sigma_{l}$ and $\sigma_{r}$ to some designated sequents in $G_{1} \mid G_{1}^{*}$.

Definition 16. The manipulation described in Step 4 is called eigenvariable-replacing operation.

Step 5. A proof $\tau^{*}$ is constructed from $\tau^{4}$ by assigning inductively one unique identification number to each occurrence of $p$ in $\tau^{4}$ as follows. 
One unique identification number, which is a positive integer, is assigned to each leaf of the form $p \Rightarrow p$ in $\tau^{4}$ which corresponds to $p_{k} \Rightarrow p_{k}$ in $\tau^{*}$. Other nodes of $\tau^{4}$ are processed as follows.

- Let $\frac{G_{1} \mid \Gamma, \lambda p \Rightarrow \mu p, \Delta}{G_{1} \mid \Gamma^{\prime}, \lambda p \Rightarrow \mu p, \Delta^{\prime}}(I) \in \tau^{4}$. Suppose that all occurrences of $p$ in $G_{1} \mid \Gamma, \lambda p \Rightarrow \mu p, \Delta$ are assigned identification numbers and have the form $G_{1}^{\prime} \mid \Gamma, p_{i_{1}}, \cdots, p_{i_{\lambda}} \Rightarrow p_{j_{1}}, \cdots, p_{j_{\mu}}, \Delta$ in $\tau^{*}$, which we often write as $G_{1}^{\prime} \mid \Gamma,\left\{p_{i_{k}}\right\}_{k=1}^{\lambda} \Rightarrow\left\{p_{j_{k}}\right\}_{k=1}^{\mu}, \Delta$. Then $G_{1} \mid \Gamma^{\prime}, \lambda p \Rightarrow \mu p, \Delta^{\prime}$ has the form $G_{1}^{\prime} \mid \Gamma^{\prime},\left\{p_{i_{k}}\right\}_{k=1}^{\lambda} \Rightarrow$ $\left\{p_{j_{k}}\right\}_{k=1}^{\mu}, \Delta^{\prime}$.

- Let $\frac{G^{\prime} G^{\prime \prime}}{G^{\prime \prime \prime}}\left(\wedge_{r w}\right) \in \tau^{4}$, where $G^{\prime} \equiv G_{1}\left|\Gamma, \lambda p \Rightarrow \mu p, A, \Delta, G^{\prime \prime} \equiv G_{2}\right| \Gamma, \lambda p \Rightarrow \mu p, B, \Delta$, $G^{\prime \prime \prime} \equiv G_{1}\left|G_{2}\right| \Gamma, \lambda p \Rightarrow \mu p, A \wedge B, \Delta \mid \Gamma, \lambda p \Rightarrow \mu p, A \wedge B, \Delta$. Suppose that $G^{\prime}$ and $G^{\prime \prime}$ have the forms $G_{1}^{\prime} \mid \Gamma,\left\{p_{i_{1 k}}\right\}_{k=1}^{\lambda} \Rightarrow\left\{p_{j_{1 k}}\right\}_{k=1}^{\mu}, A, \Delta$ and $G_{2}^{\prime} \mid \Gamma,\left\{p_{i_{2 k}}\right\}_{k=1}^{\lambda} \Rightarrow\left\{p_{j_{2 k}}\right\}_{k=1}^{\mu}, B, \Delta$ in $\tau^{*}$, respectively. Then $G^{\prime \prime \prime}$ has the form $G_{1}^{\prime}\left|G_{2}^{\prime}\right| \Gamma,\left\{p_{i_{1 k}}\right\}_{k=1}^{\lambda} \Rightarrow\left\{p_{j_{1 k}}\right\}_{k=1}^{\mu}, A \wedge B, \Delta \mid \Gamma,\left\{p_{i_{2 k}}\right\}_{k=1}^{\lambda} \Rightarrow\left\{p_{j_{2 k}}\right\}_{k=1}^{\mu}, A \wedge B, \Delta$. All applications of $\left(\vee_{l w}\right)$ are processed by the procedure similar to that of $\left(\wedge_{r w}\right)$.

- Let $\frac{G^{\prime} G^{\prime \prime}}{G^{\prime \prime \prime}}\left(\odot_{r}\right) \in \tau^{4}$, where $G^{\prime} \equiv G_{1} \mid \Gamma_{1}, \lambda_{1} p \Rightarrow \mu_{1} p, A, \Delta_{1}$,

$G^{\prime \prime} \equiv G_{2}\left|\Gamma_{2}, \lambda_{2} p \Rightarrow \mu_{2} p, B, \Delta_{2}, G^{\prime \prime \prime} \equiv G_{1}\right| G_{2} \mid \Gamma_{1}, \Gamma_{2},\left(\lambda_{1}+\lambda_{2}\right) p \Rightarrow\left(\mu_{1}+\mu_{2}\right) p, A \odot B, \Delta_{1}, \Delta_{2}$. Suppose that $G^{\prime}$ and $G^{\prime \prime}$ have the forms $G_{1}^{\prime} \mid \Gamma_{1},\left\{p_{i_{1 k}}\right\}_{k=1}^{\lambda_{1}} \Rightarrow\left\{p_{j_{1 k}}\right\}_{k=1}^{\mu_{1}}, A, \Delta_{1}$ and $G_{2}^{\prime} \mid \Gamma_{2},\left\{p_{i_{2 k}}\right\}_{k=1}^{\lambda_{2}} \Rightarrow$ $\left\{p_{j_{2 k}}\right\}_{k=1}^{\mu_{2}}, B, \Delta_{2}$ in $\tau^{*}$, respectively. Then $G^{\prime \prime \prime}$ has the form $G_{1}^{\prime}\left|G_{2}^{\prime}\right| \Gamma_{1}, \Gamma_{2},\left\{p_{i_{1 k}}\right\}_{k=1}^{\lambda_{1}},\left\{p_{i_{2 k}}\right\}_{k=1}^{\lambda_{2}} \Rightarrow$ $\left\{p_{j_{1 k}}\right\}_{k=1}^{\mu_{1}},\left\{p_{j_{2 k}}\right\}_{k=1}^{\mu_{2}}, A \odot B, \Delta_{1}, \Delta_{2}$. All applications of $\left(\rightarrow_{l}\right)$ are processed by the procedure similar to that of $\left(\odot_{r}\right)$.

- $\quad$ Let $\frac{G^{\prime} G^{\prime \prime}}{G^{\prime \prime \prime}}(C O M) \in \tau^{4}$, where $G^{\prime} \equiv G_{1} \mid \Gamma_{1}, \Pi_{1}, \lambda_{1} p \Rightarrow \mu_{1} p, \Sigma_{1}, \Delta_{1}$,

$G^{\prime \prime} \equiv G_{2}\left|\Gamma_{2}, \Pi_{2}, \lambda_{2} p \Rightarrow \mu_{2} p, \Sigma_{2}, \Delta_{2}, G^{\prime \prime \prime} \equiv G_{1}\right| G_{2}\left|\Gamma_{1}, \Gamma_{2},\left(\lambda_{11}+\lambda_{21}\right) p \Rightarrow\left(\mu_{11}+\mu_{21}\right) p, \Delta_{1}, \Delta_{2}\right|$

$\Pi_{1}, \Pi_{2},\left(\lambda_{12}+\lambda_{22}\right) p \Rightarrow\left(\mu_{12}+\mu_{22}\right) p, \Sigma_{1}, \Sigma_{2}$, where $\lambda_{11}+\lambda_{12}=\lambda_{1}, \lambda_{21}+\lambda_{22}=\lambda_{2}, \mu_{11}+\mu_{12}=$ $\mu_{1}, \mu_{21}+\mu_{22}=\mu_{2}$.

Suppose that $G^{\prime}$ and $G^{\prime \prime}$ have the forms $G_{1}^{\prime} \mid \Gamma_{1}, \Pi_{1},\left\{p_{i_{k}}\right\}_{k=1}^{\lambda_{1}} \Rightarrow\left\{p_{j_{k}^{1}}\right\}_{k=1}^{\mu_{1}}, \Sigma_{1}, \Delta_{1}$ and $G_{2}^{\prime} \mid \Gamma_{2}, \Pi_{2},\left\{p_{i_{k}^{2}}\right\}_{k=1}^{\lambda_{2}} \Rightarrow\left\{p_{j_{k}^{2}}^{2}\right\}_{k=1}^{\mu_{2}}, \Sigma_{2}, \Delta_{2}$ in $\tau^{*}$, respectively. Then $G^{\prime \prime \prime}$ has the form

$$
\begin{aligned}
& G_{1}^{\prime}\left|G_{2}^{\prime}\right| \Gamma_{1}, \Gamma_{2},\left\{p_{i_{1 k}}\right\}_{k=1}^{\lambda_{11}},\left\{p_{i_{1 k}^{2}}\right\}_{k=1}^{\lambda_{21}} \Rightarrow\left\{p_{j_{1 k}^{1}}\right\}_{k=1}^{\mu_{11}},\left\{p_{j_{1 k}^{2}}\right\}_{k=1}^{\mu_{21}}, \Delta_{1}, \Delta_{2} \mid \\
& \Pi_{1}, \Pi_{2},\left\{p_{i_{2 k}^{1}}\right\}_{k=1}^{\lambda_{12}},\left\{p_{i_{2 k}^{2}}\right\}_{k=1}^{\lambda_{22}} \Rightarrow\left\{p_{j_{2 k}^{1}}\right\}_{k=1}^{\mu_{12}},\left\{p_{j_{2 k}^{2}}\right\}_{k=1}^{\mu_{22}}, \Sigma_{1}, \Sigma_{2},
\end{aligned}
$$

where

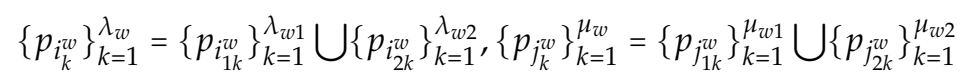

for $w=1,2$.

Definition 17. The manipulation described in Step 5 is called eigenvariable-labeling operation.

Notation 4. Let $G_{2}$ and $G_{2}^{*}$ be converted to $G$ and $G^{*}$ in $\tau^{*}$, respectively. Then $\tau^{*}$ is a proof of $G \mid G^{*}$.

In the preprocessing of $\tau$, each $\frac{G_{i}^{\prime \prime \prime} \mid\left\{S_{i}^{c}\right\}^{m_{i}^{\prime}}}{G_{i}^{\prime \prime \prime} \mid S_{i}^{c}}\left(E C^{*}\right)_{i}$ is converted into $\frac{G_{i}^{\prime \prime} \mid\left\{S_{i}^{c}\right\}^{m_{i}^{\prime}}}{G_{i}^{\prime \prime} \mid\left\{S_{i}^{c}\right\}^{m_{i}^{\prime}}}\left(I D_{\Omega}\right)_{i}$ in Step 2, where $G_{i}^{\prime \prime \prime} \subseteq G_{i}^{\prime \prime}$ by Lemma 3. $\frac{G^{\prime}}{G^{\prime} \mid S^{\prime}}(E W) \in \tau^{2}$ is converted into $\frac{G^{\prime \prime}}{G^{\prime \prime}}\left(I D_{\Omega}\right)$ in Step 3, where $G^{\prime \prime} \subseteq G^{\prime}$ by Lemma 4(i). Some $G^{\prime} \mid \Gamma^{\prime}, p \Rightarrow \Delta^{\prime} \in \tau^{3}$ (or $\left.G^{\prime} \mid \Gamma^{\prime} \Rightarrow p, \Delta^{\prime}\right)$ is revised as $G^{\prime} \mid \Gamma^{\prime}, \top \Rightarrow \Delta^{\prime}\left(\right.$ or $\left.G^{\prime} \mid \Gamma^{\prime} \Rightarrow 1, \Delta^{\prime}\right)$ in Step 4. Each occurrence of $p$ in $\tau^{4}$ is assigned the unique identification number in Step 5. The whole preprocessing above is depicted by Figure 13. 


$$
\begin{aligned}
& \stackrel{\tau}{\rightarrow} G_{0} \underset{\wedge_{r w}, \vee_{l w}}{\stackrel{\text { Step } 1: \tau^{1}}{\longrightarrow}} G_{0} \underset{\text { Step 2: } \tau^{2}}{\stackrel{\text { SC }}{\longrightarrow}} G_{0}\left|G_{0}^{*} \underset{\text { Step 3: } \tau^{3}}{\mathrm{EW}} G_{1}\right| G_{1}^{*} \\
& \stackrel{\text { Step 4: } \tau^{4}}{\longrightarrow} G_{2}\left|G_{2}^{*} \underset{\text { ID numbers }}{\stackrel{\text { Step 5: } \tau^{*}}{\longrightarrow}} G\right| G^{*}
\end{aligned}
$$

Figure 13. Preprocessing of $\tau$.

Notation 5. Let $\frac{G_{i}^{\prime \prime \prime} \mid\left\{S_{i}^{c}\right\}^{m_{i}^{\prime}}}{G_{i}^{\prime \prime \prime} \mid S_{i}^{c}}\left(E C^{*}\right)_{i}, 1 \leqslant i \leqslant N$ be all $\left(E C^{*}\right)$-nodes of $\tau^{1}$ and $G_{i}^{\prime \prime \prime} \mid\left\{S_{i}^{c}\right\}^{m_{i}^{\prime}}$ be converted to $G_{i}^{\prime \prime} \mid\left\{S_{i}^{c}\right\}^{m_{i}}$ in $\tau^{*}$. Note that there are no identification numbers for occurrences of variable $p$ in $S_{i}^{c} \in G_{i}^{\prime \prime \prime} \mid\left\{S_{i}^{c}\right\}^{m_{i}^{\prime}}$ meanwhile they are assigned to $p$ in $S_{i}^{c} \in G_{i}^{\prime \prime} \mid\left\{S_{i}^{c}\right\}^{m_{i}}$. But we use the same notations for $S_{i}^{c} \in G_{i}^{\prime \prime \prime} \mid\left\{S_{i}^{c}\right\}^{m_{i}^{\prime}}$ and $S_{i}^{c} \in G_{i}^{\prime \prime} \mid\left\{S_{i}^{c}\right\}^{m_{i}}$ for simplicity.

In the whole paper, let $H_{i}^{c}=G_{i}^{\prime} \mid\left\{S_{i}^{c}\right\}^{m_{i}}$ denote the unique node of $\tau^{*}$ such that $H_{i}^{c} \leqslant G_{i}^{\prime \prime} \mid\left\{S_{i}^{c}\right\}^{m_{i}}$ and $S_{i}^{c}$ is the focus sequent of $H_{i}^{c}$ in $\tau^{*}$, in which case we denote the focus one $S_{i 1}^{c}$ and others $S_{i 2}^{c}|\cdots| S_{i m_{i}}^{c}$ among $\left\{S_{i}^{c}\right\}^{m_{i}}$. We sometimes denote $H_{i}^{c}$ also by $G_{i}^{\prime} \mid\left\{S_{i v}^{c}\right\}_{v=1}^{m_{i}}$ or $G_{i}^{\prime}\left|S_{i 1}^{c}\right|\left\{S_{i v}^{c}\right\}_{v=2}^{m_{i}}$. We then write $G^{*}$ as $\left\{S_{i v}^{c}\right\}_{i=2 \cdots N}^{v i \cdots}$.

We call $H_{i}^{c}, S_{i u}^{c}$ the $i$-th pseudo-(EC) node of $\tau^{*}$ and pseudo-(EC) sequent, respectively. We abbreviate pseudo-EC as $p E C$. Let $H \in \tau^{*}$, by $S_{i}^{c} \in H$ we mean that $S_{i u}^{c} \in H$ for some $1 \leqslant u \leqslant m_{i}$.

It is possible that there does not exist $H_{i}^{c} \leqslant G_{i}^{\prime \prime} \mid\left\{S_{i}^{c}\right\}^{m_{i}}$ such that $S_{i}^{c}$ is the focus sequent of $H_{i}^{c}$ in $\tau^{*}$, in which case $\left\{S_{i}^{c}\right\}^{m_{i}} \subseteq G \mid G^{*}$, then it has not any effect on our argument to treat all such $S_{i}^{c}$ as members of $G$. So we assume that all $H_{i}^{c}$ are always defined for all $G_{i}^{\prime \prime} \mid\left\{S_{i}^{c}\right\}^{m_{i}}$ in $\tau^{*}$, i.e., $H_{i}^{c}>G \mid G^{*}$.

Proposition 2. (i) $\left\{S_{i v}^{c}\right\}_{v=2 \cdots m_{i}} \subseteq H$ for all $H \leqslant H_{i}^{c}$; (ii) $G^{*}=\left\{S_{i v}^{c}\right\}_{i=1 \cdots N}^{v=2 \cdots m_{i}}$.

Now, we replace locally each $\frac{G^{\prime}}{G^{\prime}}\left(I D_{\Omega}\right)$ in $\tau^{*}$ with $G^{\prime}$ and denote the resulting proof also by $\tau^{*}$, which has no essential difference with the original one, but could simplify subsequent arguments. We introduce the system $\mathbf{G L}_{\Omega}$ as follows.

Definition 18. $\mathbf{G L}_{\Omega}$ is a restricted subsystem of $\mathbf{G L}$ such that

(i) $p$ is designated as the unique eigenvariable by which we mean that it is not used to built up any formula containing logical connectives and only used as a sequent-formula.

(ii) Each occurrence of $p$ on each side of every component of a hypersequent in GL is assigned one unique identification number $i$ and written as $p_{i}$ in $\mathbf{G L}_{\Omega}$. Initial sequent $p \Rightarrow p$ of $\mathbf{G L}$ has the form $p_{i} \Rightarrow p_{i}$ in $\mathbf{G} \mathbf{L}_{\Omega}$.

(iii) Each sequent $S$ of $\mathbf{G L}$ in the form $\Gamma, \lambda p \Rightarrow \mu p, \Delta$ has the form

$$
\Gamma,\left\{p_{i_{k}}\right\}_{k=1}^{\lambda} \Rightarrow\left\{p_{j_{k}}\right\}_{k=1}^{\mu}, \Delta
$$

in $\mathbf{G L}_{\Omega}$, where $p$ does not occur in $\Gamma$ or $\Delta, i_{k} \neq i_{l}$ for all $1 \leqslant k<l \leqslant \lambda, j_{k} \neq j_{l}$ for all $1 \leqslant k<l \leqslant \mu$. Define $v_{l}(S)=\left\{i_{1}, \cdots, i_{\lambda}\right\}$ and $v_{r}(S)=\left\{j_{1}, \cdots, j_{\mu}\right\}$. Let $G$ be a hypersequent of $\mathbf{G L}_{\Omega}$ in the form $S_{1}|\cdots| S_{n}$ then $v_{l}\left(S_{k}\right) \cap v_{l}\left(S_{l}\right)=\varnothing$ and $v_{r}\left(S_{k}\right) \cap v_{r}\left(S_{l}\right)=\varnothing$ for all $1 \leqslant k<l \leqslant n$. Define $v_{l}(G)=\cup_{k=1}^{n} v_{l}\left(S_{k}\right)$, $v_{r}(G)=\cup_{k=1}^{n} v_{r}\left(S_{k}\right)$. Here, $l$ and $r$ in $v_{l}$ and $v_{r}$ indicate the left side and right side of a sequent, respectively.

(iv) A hypersequent $G$ of $\mathbf{G L}_{\Omega}$ is called closed if $v_{l}(G)=v_{r}(G)$. Two hypersequents $G^{\prime}$ and $G^{\prime \prime}$ of $\mathbf{G L}_{\Omega}$ are called disjoint if $v_{l}\left(G^{\prime}\right) \cap v_{l}\left(G^{\prime \prime}\right)=\varnothing, v_{l}\left(G^{\prime}\right) \cap v_{r}\left(G^{\prime \prime}\right)=\varnothing, v_{r}\left(G^{\prime}\right) \cap v_{l}\left(G^{\prime \prime}\right)=\varnothing$ and $v_{r}\left(G^{\prime}\right) \cap v_{r}\left(G^{\prime \prime}\right)=\varnothing$. $G^{\prime \prime}$ is a copy of $G^{\prime}$ if they are disjoint and there exist two bijections $\sigma_{l}: v_{l}\left(G^{\prime}\right) \rightarrow v_{l}\left(G^{\prime \prime}\right)$ and $\sigma_{r}: v_{r}\left(G^{\prime}\right) \rightarrow$ $v_{r}\left(G^{\prime \prime}\right)$ such that $G^{\prime \prime}$ can be obtained by applying $\sigma_{l}$ to antecedents of sequents in $G^{\prime}$ and $\sigma_{r}$ to succedents of sequents in $G^{\prime}$, i.e., $G^{\prime \prime}=\sigma_{r} \circ \sigma_{l}\left(G^{\prime}\right)$. 
(v) A closed hypersequent $G^{\prime}\left|G^{\prime \prime}\right| G^{\prime \prime \prime}$ can be contracted as $G^{\prime} \mid G^{\prime \prime}$ in $\mathbf{G L}_{\Omega}$ under the condition that $G^{\prime \prime}$ and $G^{\prime \prime \prime}$ are closed and $G^{\prime \prime \prime}$ is a copy of $G^{\prime \prime}$. We call it the constraint external contraction rule and denote it by

$$
\frac{G^{\prime}\left|G^{\prime \prime}\right| G^{\prime \prime \prime}}{G^{\prime} \mid G^{\prime \prime}}\left(E C_{\Omega}\right)
$$

Furthermore, if there do not exist two closed hypersequents $H^{\prime}, H^{\prime \prime} \subseteq G^{\prime} \mid G^{\prime \prime}$ such that $H^{\prime \prime}$ is a copy of $H^{\prime}$ then we call it the fully constraint contraction rule and denote by $\frac{G^{\prime}\left|G^{\prime \prime}\right| G^{\prime \prime \prime}}{G^{\prime} \mid G^{\prime \prime}}\left\langle E C_{\Omega}^{*}\right\rangle$.

(vi) (EW) and (CUT) of $\mathbf{G L}$ are forbidden. $(E C),\left(\wedge_{r}\right)$ and $\left(\vee_{l}\right)$ of $\mathbf{G L}$ are replaced with $\left(E C_{\Omega}\right),\left(\wedge_{r w}\right)$ and $\left(\vee_{l w}\right)$ in $\mathbf{G L}_{\Omega}$, respectively.

(vii) $G_{1} \mid S_{1}$ and $G_{2} \mid S_{2}$ are closed and disjoint for each two-premise rule $\frac{G_{1}\left|S_{1} \quad G_{2}\right| S_{2}}{G_{1}\left|G_{2}\right| H^{\prime}}(I I)$ of $\mathbf{G L}_{\Omega}$ and, $G^{\prime} \mid S^{\prime}$ is closed for each one-premise rule $\frac{G^{\prime} \mid S^{\prime}}{G^{\prime} \mid S^{\prime \prime}}(I)$.

(viii) $p$ does not occur in $\Gamma$ or $\Delta$ for each initial sequent $\Gamma, \perp \Rightarrow \Delta$ or $\Gamma \Rightarrow T, \Delta$ and, $p$ does not act as the weakening formula $A$ in $\frac{G \mid \Gamma \Rightarrow \Delta}{G \mid \Gamma \Rightarrow A, \Delta}(W R)$ or $\frac{G \mid \Gamma \Rightarrow \Delta}{G \mid \Gamma, A \Rightarrow \Delta}(W L)$ when $(W R)$ or $(W L)$ is available.

Lemma 5. Let $\tau$ be a cut-free proof of $G_{0}$ in $\mathbf{L}$ and $\tau^{*}$ be the tree resulting from preprocessing of $\tau$.

(i) If $\frac{G^{\prime} \mid S^{\prime}}{G^{\prime} \mid S^{\prime \prime}}(I) \in \tau^{*}$ then $v_{l}\left(G^{\prime} \mid S^{\prime \prime}\right)=v_{r}\left(G^{\prime} \mid S^{\prime \prime}\right)=v_{r}\left(G^{\prime} \mid S^{\prime}\right)=v_{l}\left(G^{\prime} \mid S^{\prime}\right)$;

(ii) If $\frac{G^{\prime}\left|S^{\prime} G^{\prime \prime}\right| S^{\prime \prime}}{G^{\prime}\left|G^{\prime \prime}\right| H^{\prime}}(I I) \in \tau^{*}$ then $v_{l}\left(G^{\prime}\left|G^{\prime \prime}\right| H^{\prime}\right)=v_{l}\left(G^{\prime} \mid S^{\prime}\right) \cup v_{l}\left(G^{\prime \prime} \mid S^{\prime \prime}\right)=v_{r}\left(G^{\prime}\left|G^{\prime \prime}\right| H^{\prime}\right)=$ $v_{r}\left(G^{\prime} \mid S^{\prime}\right) \cup v_{r}\left(G^{\prime \prime} \mid S^{\prime \prime}\right)$;

(iii) If $H \in \tau^{*}$ and $k \in v_{l}(H)$ then $k \in v_{r}(H)$;

(iv) If $H \in \tau^{*}$ and $k \in v_{l}(H)$ (or $k \in v_{r}(H)$ ) then $H \leqslant p_{k} \Rightarrow p_{k}$;

(v) $\tau^{*}$ is a proof of $G \mid G^{*}$ in $\mathbf{G L}_{\Omega}$ without $\left(E C_{\Omega}\right)$;

(vi) If $H^{\prime}, H^{\prime \prime} \in \tau^{*}$ and $H^{\prime} \| H^{\prime \prime}$ then $v_{l}\left(H^{\prime}\right) \cap v_{l}\left(H^{\prime \prime}\right)=\varnothing, v_{r}\left(H^{\prime}\right) \cap v_{r}\left(H^{\prime \prime}\right)=\varnothing$.

Proof. Claims from (i) to (iv) follow immediately from Step 5 in preprocessing of $\tau$ and Definition 18. Claim (v) is from Notation 4 and Definition 18. Only (vi) is proved as follows.

Suppose that $k \in v_{l}\left(H^{\prime}\right) \cap v_{l}\left(H^{\prime \prime}\right)$. Then $H^{\prime} \leqslant p_{k} \Rightarrow p_{k}, H^{\prime \prime} \leqslant p_{k} \Rightarrow p_{k}$ by Claim (iv). Thus $H^{\prime} \leqslant H^{\prime \prime}$ or $H^{\prime \prime} \leqslant H^{\prime}$, a contradiction with $H^{\prime} \| H^{\prime \prime}$ hence $v_{l}\left(H^{\prime}\right) \cap v_{l}\left(H^{\prime \prime}\right)=\varnothing$.

$v_{r}\left(H^{\prime}\right) \cap v_{r}\left(H^{\prime \prime}\right)=\varnothing$ is proved by a similar procedure and omitted.

\section{The Generalized Density Rule $(\mathcal{D})$ for $\mathrm{GL}_{\Omega}$}

In this section, we define the generalized density rule $(\mathcal{D})$ for $\mathbf{G L}_{\Omega}$ and prove that it is admissible in $\mathrm{GL}_{\Omega}$.

Definition 19. Let $G$ be a closed hypersequent of $\mathbf{G L}_{\Omega}$ and $S \in G$. Define $[S]_{G}=\bigcap\left\{H: S \in H \subseteq G, v_{l}(H)=\right.$ $\left.v_{r}(H)\right\}$, i.e., $[S]_{G}$ is the minimal closed unit of $G$ containing $S$. In general, for $G^{\prime} \subseteq G$, define $\left[G^{\prime}\right]_{G}=\cap\{H$ : $\left.G^{\prime} \subseteq H \subseteq G, v_{l}(H)=v_{r}(H)\right\}$.

Clearly, $[S]_{G}=S$ if $v_{l}(S)=v_{r}(S)$ or $p$ does not occur in $S$. The following construction gives a procedure to construct $[S]_{G}$ for any given $S \in G$.

Construction 2. Let $G$ and $S$ be as above. A sequence $G_{1}, G_{2}, \cdots, G_{n}$ of hypersequents is constructed recursively as follows. (i) $G_{1}=\{S\}$; (ii) Suppose that $G_{k}$ is constructed for $k \geqslant 1$. If $v_{l}\left(G_{k}\right) \neq v_{r}\left(G_{k}\right)$ then there exists $i_{k+1} \in v_{l}\left(G_{k}\right) \backslash v_{r}\left(G_{k}\right)$ (or $i_{k+1} \in v_{r}\left(G_{k}\right) \backslash v_{l}\left(G_{k}\right)$ ) thus there exists the unique $S_{k+1} \in G \backslash G_{k}$ such that $i_{k+1} \epsilon$ $v_{r}\left(S_{k+1}\right) \backslash v_{l}\left(S_{k+1}\right)$ (or $\left.i_{k+1} \in v_{l}\left(S_{k+1}\right) \backslash v_{r}\left(S_{k+1}\right)\right)$ by $v_{l}(G)=v_{r}(G)$ and Definition 18 then let $G_{k+1}=G_{k} \mid S_{k+1}$ otherwise the procedure terminates and $n:=k$. 
Lemma 6. (i) $G_{n}=[S]_{G}$;

(ii)Let $S^{\prime} \in[S]_{G}$ then $\left[S^{\prime}\right]_{G}=[S]_{G^{\prime}}$;

(iii)Let $G^{\prime} \equiv G\left|H^{\prime}, G^{\prime \prime} \equiv G\right| H^{\prime \prime}, v_{l}\left(G^{\prime}\right)=v_{r}\left(G^{\prime}\right), v_{l}\left(G^{\prime \prime}\right)=v_{r}\left(G^{\prime \prime}\right), v_{l}\left(H^{\prime}\right) \ominus v_{r}\left(H^{\prime}\right)=v_{l}\left(H^{\prime \prime}\right) \ominus v_{r}\left(H^{\prime \prime}\right)$ then $\left[H^{\prime}\right]_{G^{\prime}} \backslash H^{\prime}=\left[H^{\prime \prime}\right]_{G^{\prime \prime}} \backslash H^{\prime \prime}$, where $A \ominus B$ is the symmetric difference of two multisets $A, B$;

(iv)Let $v_{l r}\left(G_{k}\right)=v_{l}\left(G_{k}\right) \cap v_{r}\left(G_{k}\right)$ then $\left|v_{l r}\left(G_{k}\right)\right|+1 \geqslant\left|G_{k}\right|$ for all $1 \leqslant k \leqslant n$;

(v) $\left|v_{l}\left([S]_{G}\right)\right|+1 \geqslant\left|[S]_{G}\right|$.

Proof. (i) Since $G_{k} \subseteq G_{k+1}$ for $1 \leqslant k \leqslant n-1$ and $S \in G_{1}$ then $S \in G_{n} \subseteq G$ thus $[S]_{G} \subseteq G_{n}$ by $v_{l}\left(G_{n}\right)=v_{r}\left(G_{n}\right)$. We prove $G_{k} \subseteq[S]_{G}$ for $1 \leqslant k \leqslant n$ by induction on $k$ in the following. Clearly, $G_{1} \subseteq[S]_{G}$. Suppose that $G_{k} \subseteq[S]_{G}$ for some $1 \leqslant k \leqslant n-1$. Since $i_{k+1} \in v_{l}\left(G_{k}\right) \backslash v_{r}\left(G_{k}\right)\left(\right.$ or $\left.i_{k+1} \in v_{r}\left(G_{k}\right) \backslash v_{l}\left(G_{k}\right)\right)$ and $i_{k+1} \in v_{r}\left(S_{k+1}\right)$ (or $i_{k+1} \in v_{l}\left(S_{k+1}\right)$ ) then $S_{k+1} \in[S]_{G}$ by $G_{k} \subseteq[S]_{G}$ and $v_{l}\left([S]_{G}\right)=v_{r}\left([S]_{G}\right)$ thus $G_{k+1} \subseteq[S]_{G}$. Then $G_{n} \subseteq$ $[S]_{G}$ thus $G_{n}=[S]_{G}$.

(ii) By (i), $[S]_{G}=S_{1}\left|S_{2}\right| \cdots \mid S_{n}$, where $S_{1}=S$. Then $S^{\prime}=S_{k}$ for some $1 \leqslant k \leqslant n$ thus $i_{k} \in v_{r}\left(S_{k}\right) \backslash v_{l}\left(S_{k}\right)$ (or $i_{k} \in v_{l}\left(S_{k}\right) \backslash v_{r}\left(S_{k}\right)$ ) hence there exists the unique $k^{\prime}<k$ such that $i_{k} \in v_{l}\left(S_{k^{\prime}}\right) \backslash v_{r}\left(S_{k^{\prime}}\right)$ (or $i_{k} \in$ $\left.v_{r}\left(S_{k^{\prime}}\right) \backslash v_{l}\left(S_{k^{\prime}}\right)\right)$ if $k \geq 2$ hence $S_{k^{\prime}} \in\left[S_{k}\right]_{G}$. Repeatedly, $S_{1} \in\left[S_{k}\right]_{G^{\prime}}$, i.e., $S \in\left[S^{\prime}\right]_{G}$ then $[S]_{G} \subseteq\left[S^{\prime}\right]_{G}$. $\left[S^{\prime}\right]_{G} \subseteq[S]_{G}$ by $S^{\prime} \in[S]_{G}$ then $\left[S^{\prime}\right]_{G}=[S]_{G}$.

(iii) It holds immediately from Construction 2 and (i).

(iv) The proof is by induction on $k$. For the base step, let $k=1$ then $\left|G_{k}\right|=1$ thus $\left|v_{l r}\left(G_{k}\right)\right|+1 \geqslant\left|G_{k}\right|$ by $\left|v_{l r}\left(G_{k}\right)\right| \geqslant 0$. For the induction step, suppose that $\left|v_{l r}\left(G_{k}\right)\right|+1 \geqslant\left|G_{k}\right|$ for some $1 \leqslant k<n$. Then $\left|v_{l r}\left(G_{k+1}\right)\right| \geqslant\left|v_{l r}\left(G_{k}\right)\right|+1$ by $i_{k+1} \in v_{l r}\left(G_{k+1}\right) \backslash v_{l r}\left(G_{k}\right)$ and $v_{l r}\left(G_{k}\right) \subseteq v_{l r}\left(G_{k+1}\right)$. Then $\left|v_{l r}\left(G_{k+1}\right)\right|+1 \geqslant$ $\left|G_{k+1}\right|$ by $\left|G_{k+1}\right|=\left|G_{k}\right|+1=k+1$.

(v) It holds by (iv) and $v_{l r}\left([S]_{G}\right)=v_{l}\left([S]_{G}\right)$.

Definition 20. Let $G=S_{1}|\cdots| S_{r}$ and $S_{l}$ be in the form $\Gamma_{l},\left\{p_{i_{k}}\right\}_{k=1}^{\lambda_{l}} \Rightarrow\left\{p_{j_{k}}\right\}_{k=1}^{\mu_{l}}, \Delta_{l}$ for $1 \leqslant l \leqslant r$.

(i) If $S \in G$ and $[S]_{G}$ be $S_{k_{1}}|\cdots| S_{k_{u}}$ then $\mathcal{D}_{G}(S)$ is defined as

$\Gamma_{k_{1}}, \cdots, \Gamma_{k_{u}} \Rightarrow\left(\left|v_{l}\left([S]_{G}\right)\right|-\left|[S]_{G}\right|+1\right) t, \Delta_{k_{1}}, \cdots, \Delta_{k_{u}}$;

(ii) Let $\cup_{k=1}^{v}\left[S_{q_{k}}\right]_{G}=G$ and $\left[S_{q_{k}}\right]_{G} \cap\left[S_{q_{l}}\right]_{G}=\varnothing$ for all $1 \leqslant k<l \leqslant v$ then $\mathcal{D}(G)$ is defined as $\mathcal{D}_{G}\left(S_{q_{1}}\right)|\cdots| \mathcal{D}_{G}\left(S_{q_{v}}\right)$.

(iii) We call $(\mathcal{D})$ the generalized density rule of $\mathbf{G L}_{\Omega}$, whose conclusion $\mathcal{D}(G)$ is defined by (ii) if its premise is $G$.

Clearly, $\mathcal{D}\left(p_{k} \Rightarrow p_{k}\right)$ is $\Rightarrow t$ and $\mathcal{D}(S)=S$ if $p$ does not occur in $S$.

Lemma 7. Let $G^{\prime} \equiv G \mid S$ and $G^{\prime \prime} \equiv G\left|S_{1}\right| S_{2}$ be closed and $\left[S_{1}\right]_{G^{\prime \prime}} \cap\left[S_{2}\right]_{G^{\prime \prime}}=\varnothing$, where $S_{1}=\Gamma_{1},\left\{p_{i_{k}^{1}}\right\}_{k=1}^{\lambda_{1}} \Rightarrow$ $\left\{p_{j_{k}^{1}}\right\}_{k=1}^{\mu_{1}}, \Delta_{1} ; S_{2}=\Gamma_{2},\left\{p_{i_{k}^{2}}\right\}_{k=1}^{\lambda_{2}} \Rightarrow\left\{p_{j_{k}^{2}}\right\}_{k=1}^{\mu_{2}}, \Delta_{2} ; S=\Gamma_{1}, \Gamma_{2},\left\{p_{i_{k}^{1}}\right\}_{k=1}^{\lambda_{1}},\left\{p_{i_{k}^{2}}\right\}_{k=1}^{\lambda_{2}} \Rightarrow\left\{p_{j_{k}^{1}}\right\}_{k=1}^{\mu_{1}},\left\{p_{j_{k}^{2}}\right\}_{k=1}^{\mu_{2}}, \Delta_{1}, \Delta_{2} ;$ $\mathcal{D}_{G^{\prime \prime}}\left(S_{1}\right)=\Gamma_{1}, \Sigma_{1} \Rightarrow \Pi_{1}, \Delta_{1}$ and $\mathcal{D}_{G^{\prime \prime}}\left(S_{2}\right)=\Gamma_{2}, \Sigma_{2} \Rightarrow \Pi_{2}, \Delta_{2}$. Then $\mathcal{D}_{G^{\prime}}(S)=\Gamma_{1}, \Sigma_{1}, \Gamma_{2}, \Sigma_{2} \Rightarrow$ $\Pi_{1}, \Delta_{1}, \Pi_{2}, \Delta_{2}$.

Proof. Since $\left[S_{1}\right]_{G^{\prime \prime}} \cap\left[S_{2}\right]_{G^{\prime \prime}}=\varnothing$ then $[S]_{G^{\prime}}=\left[S_{1}\right]_{G^{\prime \prime}} \backslash\left\{S_{1}\right\} \cup\left[S_{2}\right]_{G^{\prime \prime}} \backslash\left\{S_{2}\right\} \cup\{S\}$ by $v_{l}(S)=v_{l}\left(S_{1} \mid S_{2}\right)$, $v_{r}(S)=v_{r}\left(S_{1} \mid S_{2}\right)$ and Lemma 6 (iii). Thus $\left|v_{l}\left([S]_{G^{\prime}}\right)\right|=\left|v_{l}\left(\left[S_{1}\right]_{G^{\prime \prime}}\right)\right|+\left|v_{l}\left(\left[S_{2}\right]_{G^{\prime \prime}}\right)\right|,\left|[S]_{G^{\prime}}\right|=\left|\left[S_{1}\right]_{G^{\prime \prime}}\right|+$ $\left|\left[S_{2}\right]_{G^{\prime \prime}}\right|-1$. Hence

$$
\left|v_{l}\left([S]_{G^{\prime}}\right)\right|-\left|[S]_{G^{\prime}}\right|+1=\left|v_{l}\left(\left[S_{1}\right]_{G^{\prime \prime}}\right)\right|-\left|\left[S_{1}\right]_{G^{\prime \prime}}\right|+1+\left|v_{l}\left(\left[S_{2}\right]_{G^{\prime \prime}}\right)\right|-\left|\left[S_{2}\right]_{G^{\prime \prime}}\right|+1 .
$$

Therefore $\mathcal{D}_{G^{\prime}}(S)=\Gamma_{1}, \Sigma_{1}, \Gamma_{2}, \Sigma_{2} \Rightarrow \Pi_{1}, \Delta_{1}, \Pi_{2}, \Delta_{2}$ by

$$
\begin{aligned}
\Pi_{1} & =\left(\left|v_{l}\left(\left[S_{1}\right]_{G^{\prime \prime}}\right)\right|-\left|\left[S_{1}\right]_{G^{\prime \prime}}\right|+1\right) t, \Pi_{1} \backslash\left(\left|v_{l}\left(\left[S_{1}\right]_{G^{\prime \prime}}\right)\right|-\left|\left[S_{1}\right]_{G^{\prime \prime}}\right|+1\right) t \\
\Pi_{2} & =\left(\left|v_{l}\left(\left[S_{2}\right]_{G^{\prime \prime}}\right)\right|-\left|\left[S_{2}\right]_{G^{\prime \prime}}\right|+1\right) t, \Pi_{2} \backslash\left(\left|v_{l}\left(\left[S_{2}\right]_{G^{\prime \prime}}\right)\right|-\left|\left[S_{2}\right]_{G^{\prime \prime}}\right|+1\right) t \\
\mathcal{D}_{G^{\prime}}(S) & =\Gamma_{1}, \Sigma_{1}, \Gamma_{2}, \Sigma_{2} \Rightarrow\left(\left|v_{l}\left([S]_{G^{\prime}}\right)\right|-\left|[S]_{G^{\prime}}\right|+1\right) t,
\end{aligned}
$$




$$
\Pi_{1} \backslash\left(\left|v_{l}\left(\left[S_{1}\right]_{G^{\prime \prime}}\right)\right|-\left|\left[S_{1}\right]_{G^{\prime \prime}}\right|+1\right) t, \Delta_{1}, \Pi_{2} \backslash\left(\left|v_{l}\left(\left[S_{2}\right]_{G^{\prime \prime}}\right)\right|-\left|\left[S_{2}\right]_{G^{\prime \prime}}\right|+1\right) t, \Delta_{2}
$$

where $\lambda t=\{\underbrace{t, \cdots, t}_{\lambda}\}$.

Lemma 8. (Appendix A.5.1) If there exists a proof $\tau$ of $G$ in $\mathbf{G L}_{\Omega}$ then there exists a proof of $\mathcal{D}(G)$ in $\mathbf{G} \mathbf{L}$, i.e., $(\mathcal{D})$ is admissible in $\mathbf{G L}_{\Omega}$.

Proof. We proceed by induction on the height of $\tau$. For the base step, if $G$ is $p_{k} \Rightarrow p_{k}$ then $\mathcal{D}(G)$ is $\Rightarrow t$ otherwise $\mathcal{D}(G)$ is $G$ then $\vdash_{G L} \mathcal{D}(G)$ holds. For the induction step, the following cases are considered.

Case 1 Let

$$
\frac{G^{\prime} \mid S^{\prime}}{G^{\prime} \mid S^{\prime \prime}}\left(\rightarrow_{r}\right) \in \tau
$$

where

$$
\begin{aligned}
S^{\prime} & \equiv A, \Gamma,\left\{p_{i_{k}}\right\}_{k=1}^{\lambda} \Rightarrow\left\{p_{j_{k}}\right\}_{k=1}^{\mu}, \Delta, B, \\
S^{\prime \prime} & \equiv \Gamma,\left\{p_{i_{k}}\right\}_{k=1}^{\lambda} \Rightarrow\left\{p_{j_{k}}\right\}_{k=1}^{\mu}, \Delta, A \rightarrow B .
\end{aligned}
$$

Then $\left[S^{\prime \prime}\right]_{G^{\prime} \mid S^{\prime \prime}}=\left[S^{\prime}\right]_{G^{\prime} \mid S^{\prime}} \backslash\left\{S^{\prime}\right\} \mid S^{\prime \prime}$ by $v_{l}\left(S^{\prime}\right)=v_{l}\left(S^{\prime \prime}\right), v_{r}\left(S^{\prime}\right)=v_{r}\left(S^{\prime \prime}\right)$ and Lemma 6(iii). Let $\mathcal{D}_{G^{\prime} \mid S^{\prime}}\left(S^{\prime}\right)=A, \Gamma, \Gamma^{\prime \prime} \Rightarrow \Delta^{\prime \prime}, \Delta, B$ then $\mathcal{D}_{G^{\prime} \mid S^{\prime \prime}}\left(S^{\prime \prime}\right)=\Gamma, \Gamma^{\prime \prime} \Rightarrow \Delta^{\prime \prime}, \Delta, A \rightarrow B$ thus a proof of $\mathcal{D}\left(G^{\prime} \mid S^{\prime \prime}\right)$ is constructed by combining the proof of $\mathcal{D}\left(G^{\prime} \mid S^{\prime}\right)$ and $\frac{\mathcal{D}_{G^{\prime} \mid S^{\prime}}\left(S^{\prime}\right)}{\mathcal{D}_{G^{\prime} \mid S^{\prime \prime}}\left(S^{\prime \prime}\right)}\left(\rightarrow_{r}\right)$. Other rules of type $(I)$ are processed by a procedure similar to above.

Case 2 Let

$$
\frac{G_{1}\left|S_{1} \quad G_{2}\right| S_{2}}{G_{1}\left|G_{2}\right| S_{3}}\left(\odot_{r}\right) \in \tau
$$

where

$$
\begin{gathered}
S_{1} \equiv \Gamma_{1},\left\{p_{i_{k}^{1}}\right\}_{k=1}^{\lambda_{1}} \Rightarrow\left\{p_{j_{k}^{1}}\right\}_{k=1}^{\mu_{1}}, A, \Delta_{1} \\
S_{2} \equiv \Gamma_{2},\left\{p_{i_{k}^{2}}\right\}_{k=1}^{\lambda_{2}} \Rightarrow\left\{p_{j_{k}^{2}}\right\}_{k=1}^{\mu_{2}}, B, \Delta_{2} \\
S_{3} \equiv \Gamma_{1}, \Gamma_{2},\left\{p_{i_{k}}\right\}_{k=1^{\prime}}^{\lambda_{1}}\left\{p_{i_{k}^{2}}\right\}_{k=1}^{\lambda_{2}} \Rightarrow\left\{p_{j_{k}^{2}}\right\}_{k=1}^{\mu_{2}},\left\{p_{j_{k}^{1}}\right\}_{k=1}^{\mu_{1}}, A \odot B, \Delta_{1}, \Delta_{2} .
\end{gathered}
$$

Let

$$
\begin{aligned}
& \mathcal{D}_{G_{1} \mid S_{1}}\left(S_{1}\right)=\Gamma_{1}, \Gamma_{11} \Rightarrow \Delta_{11},\left(\left|v_{l}\left(\left[S_{1}\right]_{G_{1} \mid S_{1}}\right)\right|-\left|\left[S_{1}\right]_{G_{1} \mid S_{1}}\right|+1\right) t, A, \Delta_{1}, \\
& \mathcal{D}_{G_{2} \mid S_{2}}\left(S_{2}\right)=\Gamma_{2}, \Gamma_{21} \Rightarrow \Delta_{21},\left(\left|v_{l}\left(\left[S_{2}\right]_{G_{2} \mid S_{2}}\right)\right|-\left|\left[S_{2}\right]_{G_{2} \mid S_{2}}\right|+1\right) t, B, \Delta_{2} .
\end{aligned}
$$

Then $\mathcal{D}_{G_{1}\left|G_{2}\right| S_{3}}\left(S_{3}\right)$ is

$$
\begin{gathered}
\Gamma_{1}, \Gamma_{2}, \Gamma_{11}, \Gamma_{21} \Rightarrow \Delta_{11}, \Delta_{21}, A \odot B, \Delta_{1}, \Delta_{2} \\
\left(\left|v_{l}\left(\left[S_{1}\right]_{G_{1} \mid S_{1}}\right)\right|+\left|v_{l}\left(\left[S_{2}\right]_{G_{2} \mid S_{2}}\right)\right|-\left|\left[S_{1}\right]_{G_{1} \mid S_{1}}\right|-\left|\left[S_{2}\right]_{G_{2} \mid S_{2}}\right|+2\right) t
\end{gathered}
$$

by $\left[S_{3}\right]_{G_{1}\left|G_{2}\right| S_{3}}=\left(\left[S_{1}\right]_{G_{1} \mid S_{1}} \backslash\left\{S_{1}\right\}\right) \cup\left(\left[S_{2}\right]_{G_{2} \mid S_{2}} \backslash\left\{S_{2}\right\}\right) \cup\left\{S_{3}\right\}$. Then the proof of $\mathcal{D}\left(G_{1}\left|G_{2}\right| S_{3}\right)$ is constructed by combining $\vdash_{\mathrm{GL}} \mathcal{D}\left(G_{1} \mid S_{1}\right)$ and

$\vdash_{\text {GL }} \mathcal{D}\left(G_{2} \mid S_{2}\right)$ with $\frac{\mathcal{D}_{G_{1} \mid S_{1}}\left(S_{1}\right) \mathcal{D}_{G_{2} \mid S_{2}}\left(S_{2}\right)}{\mathcal{D}_{G_{1}\left|G_{2}\right| S_{3}}\left(S_{3}\right)}\left(\odot_{r}\right)$. All applications of $\left(\rightarrow_{l}\right)$ are processed by a procedure similar to that of $\odot_{r}$ and omitted.

Case 3 Let

$$
\frac{G^{\prime} G^{\prime \prime}}{G^{\prime \prime \prime}}\left(\wedge_{r w}\right) \in \tau
$$

where

$$
G^{\prime} \equiv G_{1}\left|S_{1}, \quad G^{\prime \prime} \equiv G_{2}\right| S_{2}, \quad G^{\prime \prime \prime} \equiv G_{1}\left|G_{2}\right| S_{1}^{\prime} \mid S_{2}^{\prime}
$$




$$
\begin{gathered}
S_{w} \equiv \Gamma_{w},\left\{p_{i_{k}^{w}}\right\}_{k=1}^{\lambda_{w}} \Rightarrow\left\{p_{j_{k}^{w}}\right\}_{k=1}^{\mu_{w}}, A_{w}, \Delta_{w}, \\
S_{w}^{\prime} \equiv \Gamma_{w},\left\{p_{i_{k}^{w}}\right\}_{k=1}^{\lambda_{w}} \Rightarrow\left\{p_{j_{k}^{w}}\right\}_{k=1}^{\mu_{w}}, A_{1} \wedge A_{2}, \Delta_{w}
\end{gathered}
$$

for $w=1$, 2. Then $\left[S_{1}^{\prime}\right]_{G^{\prime \prime \prime}}=\left[S_{1}\right]_{G^{\prime}} \backslash\left\{S_{1}\right\}\left|S_{1}^{\prime},\left[S_{2}^{\prime}\right]_{G^{\prime \prime \prime}}=\left[S_{2}\right]_{G^{\prime \prime}} \backslash\left\{S_{2}\right\}\right| S_{2}^{\prime}$ by Lemma 6 (iii). Let

$$
\mathcal{D}_{G_{w} \mid S_{w}}\left(S_{w}\right)=\Gamma_{w}, \Gamma_{w 1} \Rightarrow \Delta_{w 1},\left(\left|v_{l}\left(\left[S_{w}\right]_{G_{w} \mid S_{w}}\right)\right|-\left|\left[S_{w}\right]_{G_{w} \mid S_{w}}\right|+1\right) t, A_{w}, \Delta_{1}
$$

for $w=1,2$. Then

$$
\mathcal{D}_{G^{\prime \prime \prime}}\left(S_{w}^{\prime}\right)=\Gamma_{w}, \Gamma_{w 1} \Rightarrow \Delta_{w 1},\left(\left|v_{l}\left(\left[S_{w}\right]_{G_{w} \mid S_{w}}\right)\right|-\left|\left[S_{w}\right]_{G_{w} \mid S_{w}}\right|+1\right) t, A_{1} \wedge A_{2}, \Delta_{w}
$$

for $w=1$, 2. Then the proof of $\mathcal{D}\left(G^{\prime \prime \prime}\right)$ is constructed by combining $\vdash_{\mathrm{GL}} \mathcal{D}\left(G^{\prime}\right)$ and $\vdash_{\mathrm{GL}} \mathcal{D}\left(G^{\prime \prime}\right)$ with $\frac{\mathcal{D}_{G^{\prime}}\left(S_{1}\right) \mathcal{D}_{G^{\prime \prime}}\left(S_{2}\right)}{\mathcal{D}_{G^{\prime \prime \prime}}\left(S_{1}^{\prime} \mid S_{2}^{\prime}\right)}\left(\wedge_{r w w}\right)$. All applications of $\left(\vee_{l w}\right)$ are processed by a procedure similar to that of $\left(\wedge_{r w}\right)$ and omitted.

\section{Case 4 Let}

$$
\frac{G^{\prime} G^{\prime \prime}}{G^{\prime \prime \prime}}(C O M) \in \tau
$$

where

$$
\begin{gathered}
G^{\prime} \equiv G_{1}\left|S_{1}, \quad G^{\prime \prime} \equiv G_{2}\right| S_{2}, \quad G^{\prime \prime \prime} \equiv G_{1}\left|G_{2}\right| S_{3} \mid S_{4} \\
S_{1} \equiv \Gamma_{1}, \Pi_{1},\left\{p_{i_{k}^{1}}\right\}_{k=1}^{\lambda_{1}} \Rightarrow\left\{p_{j_{k}^{1}}\right\}_{k=1}^{\mu_{1}}, \Sigma_{1}, \Delta_{1}, \\
S_{2} \equiv \Gamma_{2}, \Pi_{2},\left\{p_{i_{k}^{2}}\right\}_{k=1}^{\lambda_{2}} \Rightarrow\left\{p_{j_{k}^{2}}\right\}_{k=1}^{\mu_{2}}, \Sigma_{2}, \Delta_{2}, \\
S_{3} \equiv \Gamma_{1}, \Gamma_{2},\left\{p_{i_{1 k}^{1}}\right\}_{k=1}^{\lambda_{11}},\left\{p_{i_{1 k}^{2}}\right\}_{k=1}^{\lambda_{21}} \Rightarrow\left\{p_{j_{1 k}^{1}}\right\}_{k=1}^{\mu_{11}},\left\{p_{j_{1 k}^{2}}\right\}_{k=1}^{\mu_{21}}, \Delta_{1}, \Delta_{2}, \\
S_{4} \equiv \Pi_{1}, \Pi_{2},\left\{p_{i_{2 k}^{1}}\right\}_{k=1}^{\lambda_{12}},\left\{p_{i_{2 k}^{2}}\right\}_{k=1}^{\lambda_{22}} \Rightarrow\left\{p_{j_{2 k}}\right\}_{k=1}^{\mu_{12}}\left\{p_{j_{2 k}}\right\}_{k=1}^{\mu_{22}}, \Sigma_{1}, \Sigma_{2}
\end{gathered}
$$

where $\left\{p_{i_{k}^{w}}\right\}_{k=1}^{\lambda_{w}}=\left\{p_{i_{k} w}\right\}_{k=1}^{\lambda_{w 1}} \cup\left\{p_{i_{2 k}^{w}}\right\}_{k=1}^{\lambda_{w 2}},\left\{p_{j_{k}^{w}}\right\}_{k=1}^{\mu_{w}}=\left\{p_{j_{1 k}}\right\}_{k=1}^{\mu_{w 1}} \cup\left\{p_{j_{2 k}}\right\}_{k=1}^{\mu_{w 22}}$ for $w=1,2$

Case 4.1. $S_{3} \in\left[S_{4}\right]_{G^{\prime \prime \prime}}$. Then $\left[S_{3}\right]_{G^{\prime \prime \prime}}=\left[S_{4}\right]_{G^{\prime \prime \prime}}$ by Lemma 6(ii) and $\left[S_{3}\right]_{G^{\prime \prime \prime}}=\left[S_{1}\right]_{G^{\prime}}\left|\left[S_{2}\right]_{G^{\prime \prime}}\right| S_{3} \mid S_{4} \backslash\left\{S_{1}, S_{2}\right\}$ by Lemma 6(iii). Then

$$
\left|v_{l}\left(\left[S_{3}\right]_{G^{\prime \prime \prime}}\right)\right|-\left|\left[S_{3}\right]_{G^{\prime \prime \prime}}\right|+1=\left|v_{l}\left(\left[S_{1}\right]_{G^{\prime}}\right)\right|+\left|v_{l}\left(\left[S_{2}\right]_{G^{\prime \prime}}\right)\right|-\left|\left[S_{1}\right]_{G^{\prime}}\right|-\left|\left[S_{2}\right]_{G^{\prime \prime}}\right|+1 \geqslant 0 .
$$

Thus $\left|v_{l}\left(\left[S_{1}\right]_{G^{\prime}}\right)\right|-\left|\left[S_{1}\right]_{G^{\prime}}\right|+1 \geqslant 1$ or $\left|v_{l}\left(\left[S_{2}\right]_{G^{\prime \prime}}\right)\right|-\left|\left[S_{2}\right]_{G^{\prime \prime}}\right|+1 \geqslant 1$. Hence we assume that, without loss of generality,

$$
\begin{aligned}
& \mathcal{D}_{G^{\prime}}\left(S_{1}\right)=\Gamma_{1}, \Pi_{1}, \Gamma^{\prime} \Rightarrow \Delta^{\prime}, t, \Sigma_{1}, \Delta_{1}, \\
& \mathcal{D}_{G^{\prime \prime}}\left(S_{2}\right)=\Gamma_{2}, \Pi_{2}, \Gamma^{\prime \prime} \Rightarrow \Delta^{\prime \prime}, \Sigma_{2}, \Delta_{2} .
\end{aligned}
$$

Then

$$
\mathcal{D}_{G^{\prime \prime \prime}}\left(S_{3} \mid S_{4}\right)=\Gamma_{1}, \Pi_{1}, \Gamma^{\prime}, \Gamma_{2}, \Pi_{2}, \Gamma^{\prime \prime} \Rightarrow \Delta^{\prime}, \Sigma_{1}, \Delta_{1}, \Delta^{\prime \prime}, \Sigma_{2}, \Delta_{2}
$$

Thus the proof of $\frac{\mathcal{D}_{G^{\prime}}\left(S_{1}\right) \mathcal{D}_{G^{\prime \prime}}\left(S_{2}\right)}{\mathcal{D}_{G^{\prime \prime \prime}}\left(S_{3} \mid S_{4}\right)}$ is constructed by

$$
\frac{\Gamma_{1}, \Pi_{1}, \Gamma^{\prime} \Rightarrow \Delta^{\prime}, t, \Sigma_{1}, \Delta_{1} \frac{\Gamma_{2}, \Pi_{2}, \Gamma^{\prime \prime} \Rightarrow \Delta^{\prime \prime}, \Sigma_{2}, \Delta_{2}}{\Gamma_{2}, \Pi_{2}, \Gamma^{\prime \prime}, t \Rightarrow \Delta^{\prime \prime}, \Sigma_{2}, \Delta_{2}}\left(t_{l}\right)}{\Gamma_{1}, \Pi_{1}, \Gamma^{\prime}, \Gamma_{2}, \Pi_{2}, \Gamma^{\prime \prime} \Rightarrow \Delta^{\prime}, \Sigma_{1}, \Delta_{1}, \Delta^{\prime \prime}, \Sigma_{2}, \Delta_{2}}(\text { CUT }) .
$$

Case 4.2. $S_{3} \notin\left[S_{4}\right]_{G^{\prime \prime \prime}}$. Then $\left[S_{3}\right]_{G^{\prime \prime \prime}} \cap\left[S_{4}\right]_{G^{\prime \prime \prime}}=\varnothing$ by Lemma 6(ii). Let

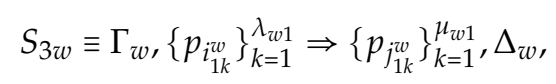




$$
S_{4 w} \equiv \Pi_{w},\left\{p_{i w}^{w}\right\}_{k=1}^{\lambda_{w 2}} \Rightarrow\left\{p_{j k}\right\}_{k=1}^{\lambda_{w 2}}, \Sigma_{w}
$$

for $w=1,2$. Then

$$
\begin{aligned}
& {\left[S_{3}\right]_{G^{\prime \prime \prime}}=\left[S_{31}\right]_{G_{1}\left|S_{31}\right| S_{41}} \backslash\left\{S_{31}\right\} \bigcup\left[S_{32}\right]_{G_{2}\left|S_{32}\right| S_{42}} \backslash\left\{S_{32}\right\} \bigcup\left\{S_{3}\right\},} \\
& {\left[S_{4}\right]_{G^{\prime \prime \prime}}=\left[S_{41}\right]_{G_{1}\left|S_{31}\right| S_{41}} \backslash\left\{S_{41}\right\} \bigcup\left[S_{42}\right]_{G_{2}\left|S_{32}\right| S_{42}} \backslash\left\{S_{42}\right\} \bigcup\left\{S_{4}\right\}}
\end{aligned}
$$

by $v_{l}\left(S_{3}\right)=v_{l}\left(S_{31} \mid S_{32}\right), v_{l}\left(S_{1}\right)=v_{l}\left(S_{31} \mid S_{41}\right), v_{l}\left(S_{2}\right)=v_{l}\left(S_{32} \mid S_{42}\right)$ and $v_{l}\left(S_{4}\right)=v_{l}\left(S_{41} \mid S_{42}\right)$. Let

$$
\begin{aligned}
& \mathcal{D}_{G_{w}\left|S_{3 w}\right| S_{4 w}}\left(S_{3 w}\right)=\Gamma_{w}, \mathrm{X}_{3 w} \Rightarrow \Psi_{3 w}, \Delta_{w}, \\
& \mathcal{D}_{G_{w}\left|S_{3 w}\right| S_{4 w}}\left(S_{4 w}\right)=\Pi_{w}, \mathrm{X}_{4 w} \Rightarrow \Psi_{4 w}, \Sigma_{w}
\end{aligned}
$$

for $w=1,2$. Then

$$
\begin{aligned}
& \mathcal{D}_{G^{\prime}}\left(S_{1}\right)=\Gamma_{1}, \Pi_{1}, X_{31}, X_{41} \Rightarrow \Psi_{31}, \Psi_{41}, \Sigma_{1}, \Delta_{1}, \\
& \mathcal{D}_{G^{\prime \prime}}\left(S_{2}\right)=\Gamma_{2}, \Pi_{2}, X_{32}, X_{42} \Rightarrow \Psi_{32}, \Psi_{42}, \Sigma_{2}, \Delta_{2}, \\
& \mathcal{D}_{G^{\prime \prime \prime}}\left(S_{3}\right)=\Gamma_{1}, X_{31}, \Gamma_{2}, X_{32} \Rightarrow \Psi_{31}, \Delta_{1}, \Psi_{32}, \Delta_{2}, \\
& \mathcal{D}_{G^{\prime \prime \prime}}\left(S_{4}\right)=\Pi_{1}, X_{41}, \Pi_{2}, X_{42} \Rightarrow \Psi_{41}, \Sigma_{1}, \Psi_{42}, \Sigma_{2}
\end{aligned}
$$

by Lemma 7, $\left[S_{3}\right]_{G^{\prime \prime \prime}} \cap\left[S_{4}\right]_{G^{\prime \prime \prime}}=\varnothing,\left[S_{31}\right]_{G_{1}\left|S_{31}\right| S_{41}} \cap\left[S_{41}\right]_{G_{1}\left|S_{31}\right| S_{41}}=\varnothing,\left[S_{32}\right]_{G_{2}\left|S_{32}\right| S_{42}} \cap\left[S_{42}\right]_{G_{2}\left|S_{32}\right| S_{42}}=\varnothing$. Then the proof of $\mathcal{D}_{G^{\prime \prime \prime}}\left(S_{3} \mid S_{4}\right)$ is constructed by combing the proofs of $\mathcal{D}_{G^{\prime}}\left(S_{1}\right)$ and $\mathcal{D}_{G^{\prime \prime}}\left(S_{2}\right)$ with $\frac{\mathcal{D}_{G^{\prime}}\left(S_{1}\right) \mathcal{D}_{G^{\prime \prime}}\left(S_{2}\right)}{\mathcal{D}_{G^{\prime \prime \prime}}\left(S_{3} \mid S_{4}\right)}(C O M)$.

Case $5 \frac{G^{\prime}\left|G^{\prime \prime}\right| G^{\prime \prime \prime}}{G^{\prime} \mid G^{\prime \prime}}\left(E C_{\Omega}\right) \in \tau$. Then $G^{\prime}, G^{\prime \prime}$ and $G^{\prime \prime \prime}$ are closed and $G^{\prime \prime \prime}$ is a copy of $G^{\prime \prime}$ thus $\mathcal{D}_{G^{\prime}\left|G^{\prime \prime}\right| G^{\prime \prime \prime}}\left(G^{\prime \prime}\right)=\mathcal{D}_{G^{\prime}\left|G^{\prime \prime}\right| G^{\prime \prime \prime}}\left(G^{\prime \prime \prime}\right)$ hence a proof of $\mathcal{D}\left(G^{\prime} \mid G^{\prime \prime}\right)$ is constructed by combining the proof of $\mathcal{D}\left(G^{\prime}\left|G^{\prime \prime}\right| G^{\prime \prime \prime}\right)$ and $\frac{\mathcal{D}\left(G^{\prime}\left|G^{\prime \prime}\right| G^{\prime \prime \prime}\right)}{\mathcal{D}\left(G^{\prime} \mid G^{\prime \prime}\right)}\left(E C^{*}\right)$.

The following two lemmas are corollaries of Lemma 8.

Lemma 9. If there exists a derivation of $G_{0}$ from $G_{1}, \cdots, G_{r}$ in $\mathbf{G L}_{\Omega}$ then there exists a derivation of $\mathcal{D}\left(G_{0}\right)$ from $\mathcal{D}\left(G_{1}\right), \cdots, \mathcal{D}\left(G_{r}\right)$ in $\mathbf{G L}$.

Lemma 10. Let $\tau$ be a cut-free proof of $G_{0}$ in $\mathbf{G L}$ and $\tau^{*}$ be the proof of $G \mid G^{*}$ in $\mathbf{G L}_{\Omega}$ resulting from preprocessing of $\tau$. Then $\vdash \mathrm{GL} \mathcal{D}\left(G \mid G^{*}\right)$.

\section{Extraction of Elimination Rules}

In this section, we will investigate Construction 1 further to extract more derivations from $\tau^{*}$.

Any two sequents in a hypersequent seem independent of one another in the sense that they can only be contracted into one by $(E C)$ when it is applicable. Note that one-premise logical rules just modify one sequent of a hypersequent and two-premise rules associate a sequent in a hypersequent with one in a different hypersequent.

$\tau^{*}$ (or any proof without $\left(E C_{\Omega}\right)$ in $\mathrm{GL}_{\Omega}$ ) has an essential property, which we call the distinguishability of $\tau^{*}$, i.e., any variables, formulas, sequents or hypersequents which occur at the node $H$ of $\tau^{*}$ occur inevitably at $H^{\prime}<H$ in some forms.

Let $H \equiv G^{\prime}\left|S^{\prime}\right| S^{\prime \prime} \in \tau^{*}$. If $S^{\prime}$ is equal to $S^{\prime \prime}$ as two sequents then the case that $\tau_{H: S^{\prime}}^{*}$ is equal to $\tau_{H: S^{\prime \prime}}^{*}$ as two derivations could possibly happen. This means that both $S^{\prime}$ and $S^{\prime \prime}$ are the focus sequent of one node in $\tau^{*}$ when $G_{H: S^{\prime}}^{*} \neq S^{\prime}$ and $G_{H: S^{\prime \prime}}^{*} \neq S^{\prime \prime}$, which contradicts that each node has the unique focus sequent in any derivation. Thus we need to differentiate $S^{\prime}$ from $S^{\prime \prime}$ for all $G^{\prime}\left|S^{\prime}\right| S^{\prime \prime} \in \tau^{*}$. 
Define $\overline{S^{\prime}} \in \tau^{*}$ such that $G^{\prime}\left|S^{\prime}\right| S^{\prime \prime} \leqslant \overline{S^{\prime}}, S^{\prime} \in \overline{S^{\prime}}$ and $S^{\prime}$ is the principal sequent of $\overline{S^{\prime}}$. If $\overline{S^{\prime}}$ has the unique principal sequent, $N_{S^{\prime}}:=0$, otherwise $N_{S^{\prime}}:=1$ (or $N_{S^{\prime}}=2$ ) to indicate that $S^{\prime}$ is one designated principal sequent (or accordingly $N_{S^{\prime}}=2$ for another) of such an application as $(C O M),\left(\wedge_{r w}\right)$ or $\left(\vee_{l w}\right)$. Then we may regard $S^{\prime}$ as $\left(S^{\prime} ; \mathcal{P}\left(\overline{S^{\prime}}\right), N_{S^{\prime}}\right)$. Thus $S^{\prime}$ is always different from $S^{\prime \prime}$ by $\mathcal{P}\left(\overline{S^{\prime}}\right) \neq \mathcal{P}\left(\overline{S^{\prime \prime}}\right)$ or, $\mathcal{P}\left(\overline{S^{\prime}}\right)=\mathcal{P}\left(\overline{S^{\prime \prime}}\right)$ and $N_{S^{\prime}} \neq N_{S^{\prime \prime}}$. We formulate it by the following construction.

Construction 3. (Appendix A.5.2) A labeled tree $\tau^{* *}$, which has the same tree structure as $\tau^{*}$, is constructed as follows.

(i) If $S$ is a leaf $\tau^{*}$, define $\bar{S}=S, N_{S}=0$ and the node $\mathcal{P}(S)$ of $\tau^{* *}$ is labeled by $\left(S ; \mathcal{P}(\bar{S}), N_{S}\right)$;

(ii) If $\frac{G^{\prime} \mid S^{\prime}}{H \equiv G^{\prime} \mid S^{\prime \prime}}(I) \in \tau^{*}$ and $\mathcal{P}\left(G^{\prime} \mid S^{\prime}\right)$ be labeled by $\mathcal{G}^{\prime} \mid\left(S^{\prime} ; \mathcal{P}\left(\overline{S^{\prime}}\right), N_{S^{\prime}}\right)$ in $\tau^{* *}$. Then define $\overline{S^{\prime \prime}}=H$, $N_{S^{\prime \prime}}=0$ and the node $\mathcal{P}(H)$ of $\tau^{* *}$ is labeled by $\mathcal{G}^{\prime} \mid\left(S^{\prime \prime} ; \mathcal{P}\left(\overline{S^{\prime \prime}}\right), N_{S^{\prime \prime}}\right)$;

(iii) If $\frac{G^{\prime}\left|S^{\prime} \quad G^{\prime \prime}\right| S^{\prime \prime}}{H \equiv G^{\prime}\left|G^{\prime \prime}\right| H^{\prime}}(I I) \in \tau^{*}, \mathcal{P}\left(G^{\prime} \mid S^{\prime}\right)$ and $\mathcal{P}\left(G^{\prime \prime} \mid S^{\prime \prime}\right)$ be labeled by $\mathcal{G}^{\prime} \mid\left(S^{\prime} ; \mathcal{P}\left(\overline{S^{\prime}}\right), N_{S^{\prime}}\right)$ and $\mathcal{G}^{\prime \prime} \mid\left(S^{\prime \prime} ; \mathcal{P}\left(\overline{S^{\prime \prime}}\right), N_{S^{\prime \prime}}\right)$ in $\tau^{* *}$, respectively. If $H^{\prime}=S_{1} \mid S_{2}$ then define $\overline{S_{1}}=\overline{S_{2}}=H, N_{S_{1}}=1, N_{S_{2}}=2$ and the node $\mathcal{P}(H)$ of $\tau^{* *}$ is labeled by $\mathcal{G}^{\prime}\left|\mathcal{G}^{\prime \prime}\right|\left(S_{1} ; \mathcal{P}\left(\overline{S_{1}}\right), N_{S_{1}}\right) \mid\left(S_{2} ; \mathcal{P}\left(\overline{S_{2}}\right), N_{S_{2}}\right)$. If $H^{\prime}=S_{1}$ then define $\overline{S_{1}}=H$, $N_{S_{1}}=0$ and $\mathcal{P}(H)$ is labeled by $\mathcal{G}^{\prime}\left|\mathcal{G}^{\prime \prime}\right|\left(S_{1} ; \mathcal{P}\left(\overline{S_{1}}\right), N_{S_{1}}\right)$.

In the whole paper, we treat $\tau^{*}$ as $\tau^{* *}$ without mention of $\tau^{* *}$. Note that in preprocessing of $\tau$, some logical applications could also be converted to $\left(I D_{\Omega}\right)$ in Step 3 and we need fix the focus sequent at each node $H$ and subsequently assign valid identification numbers to each $H^{\prime}<H$ by eigenvariable-labeling operation.

Proposition 3. (i) $G^{\prime}\left|S^{\prime}\right| S^{\prime \prime} \in \tau^{*}$ implies $\left\{S^{\prime}\right\} \cap\left\{S^{\prime \prime}\right\}=\varnothing$; (ii) $H \in \tau^{*}$ and $H^{\prime} \mid H^{\prime \prime} \subseteq H$ imply $H^{\prime} \cap H^{\prime \prime}=\varnothing$; (iii) Let $H \in \tau^{*}$ and $S_{i}^{c} \in H$ then $H \leqslant H_{i}^{c}$ or $H_{i}^{c} \leqslant H$.

Proof. (iii) Let $S_{i}^{c} \in H$ then $S_{i}^{c}=S_{i u}^{c}$ for some $1 \leqslant u \leqslant m_{i}$ by Notation 5 . Thus $S_{i}^{c} \in H_{i}^{c}$ also by Notation 5 . Hence $H \leqslant \overline{S_{i}^{c}}$ and $H_{i}^{c} \leqslant \overline{S_{i}^{c}}$ by Construction 3. Therefore $H \leqslant H_{i}^{c}$ or $H_{i}^{c} \leqslant H$.

Lemma 11. Let $H \in \tau^{*}$ and $\operatorname{Th}(H)=\left(H_{0}, \cdots, H_{n}\right)$, where $H_{0}=H, H_{n}=G \mid G^{*}, G_{k} \subseteq H$ for $1 \leqslant k \leqslant 3$.

(i) If $G_{3}=G_{1} \cap G_{2}$ then $\left\langle H_{i}\right\rangle_{H: G_{3}}=\left\langle H_{i}\right\rangle_{H: G_{1}} \cap\left\langle H_{i}\right\rangle_{H: G_{2}}$ for all $0 \leqslant i \leqslant n$;

(ii) If $G_{3}=G_{1} \mid G_{2}$ then $\left\langle H_{i}\right\rangle_{H: G_{3}}=\left\langle H_{i}\right\rangle_{H: G_{1}} \mid\left\langle H_{i}\right\rangle_{H: G_{2}}$ for all $0 \leqslant i \leqslant n$.

Proof. The proof is by induction on $i$ for $0 \leqslant i<n$. Only (i) is proved as follows and (ii) by a similar procedure and omitted.

For the base step, $\left\langle H_{0}\right\rangle_{H: G_{3}}=\left\langle H_{0}\right\rangle_{H: G_{1}} \cap\left\langle H_{0}\right\rangle_{H: G_{2}}$ holds by $\left\langle H_{0}\right\rangle_{H: G_{1}}=G_{1},\left\langle H_{0}\right\rangle_{H: G_{2}}=G_{2}$, $\left\langle H_{0}\right\rangle_{H: G_{3}}=G_{3}$ and $G_{3}=G_{1} \cap G_{2}$.

For the induction step, suppose that $\left\langle H_{i}\right\rangle_{H: G_{3}}=\left\langle H_{i}\right\rangle_{H: G_{1}} \cap\left\langle H_{i}\right\rangle_{H: G_{2}}$ for some $0 \leqslant i<n$. Only is the case of a one-premise rule given in the following and other cases are omitted.

Let $\frac{G^{\prime} \mid S^{\prime}}{G^{\prime} \mid S^{\prime \prime}}(I) \in \tau^{*}, H_{i}=G^{\prime} \mid S^{\prime}$ and $H_{i+1}=G^{\prime} \mid S^{\prime \prime}$.

Let $S^{\prime} \in\left\langle H_{i}\right\rangle_{H: G_{3}}$. Then $\left\langle H_{i+1}\right\rangle_{H: G_{3}}=\left(\left\langle H_{i}\right\rangle_{H: G_{3}} \backslash\left\{S^{\prime}\right\}\right)\left|S^{\prime \prime},\left\langle H_{i+1}\right\rangle_{H: G_{1}}=\left(\left\langle H_{i}\right\rangle_{H: G_{1}} \backslash\left\{S^{\prime}\right\}\right)\right| S^{\prime \prime}$ by $S^{\prime} \in\left\langle H_{i}\right\rangle_{H: G_{1}}$ and

$$
\left\langle H_{i+1}\right\rangle_{H: G_{2}}=\left(\left\langle H_{i}\right\rangle_{H: G_{2}} \backslash\left\{S^{\prime}\right\}\right) \mid S^{\prime \prime} \text { by } S^{\prime} \in\left\langle H_{i}\right\rangle_{H: G_{2}} .
$$

Thus

$$
\left\langle H_{i+1}\right\rangle_{H: G_{3}}=\left\langle H_{i+1}\right\rangle_{H: G_{1}} \cap\left\langle H_{i+1}\right\rangle_{H: G_{2}} \text { by }\left\langle H_{i}\right\rangle_{H: G_{3}}=\left\langle H_{i}\right\rangle_{H: G_{1}} \cap\left\langle H_{i}\right\rangle_{H: G_{2}} .
$$

Let $S^{\prime} \notin\left\langle H_{i}\right\rangle_{H: G_{1}}$ and $S^{\prime} \notin\left\langle H_{i}\right\rangle_{H: G_{2}}$. Then $\left\langle H_{i+1}\right\rangle_{H: G_{1}}=\left\langle H_{i}\right\rangle_{H: G_{1}}$,

$$
\left\langle H_{i+1}\right\rangle_{H: G_{2}}=\left\langle H_{i}\right\rangle_{H: G_{2}} \text { and }\left\langle H_{i+1}\right\rangle_{H: G_{3}}=\left\langle H_{i}\right\rangle_{H: G_{3}} \text {. }
$$

Thus 


$$
\left\langle H_{i+1}\right\rangle_{H: G_{3}}=\left\langle H_{i+1}\right\rangle_{H: G_{1}} \cap\left\langle H_{i+1}\right\rangle_{H: G_{2}} \text { by }\left\langle H_{i}\right\rangle_{H: G_{3}}=\left\langle H_{i}\right\rangle_{H: G_{1}} \cap\left\langle H_{i}\right\rangle_{H: G_{2}} .
$$

Let $S^{\prime} \notin\left\langle H_{i}\right\rangle_{H: G_{1}}, S^{\prime} \in\left\langle H_{i}\right\rangle_{H: G_{2}}$. Then $\left\langle H_{i+1}\right\rangle_{H: G_{1}}=\left\langle H_{i}\right\rangle_{H: G_{1}}$,

$$
\left\langle H_{i+1}\right\rangle_{H: G_{3}}=\left\langle H_{i}\right\rangle_{H: G_{3}} \text { and }\left\langle H_{i+1}\right\rangle_{H: G_{2}}=\left(\left\langle H_{i}\right\rangle_{H: G_{2}} \backslash\left\{S^{\prime}\right\}\right) \mid S^{\prime \prime} \text {. }
$$

Thus

$$
\left\langle H_{i+1}\right\rangle_{H: G_{3}}=\left\langle H_{i+1}\right\rangle_{H: G_{1}} \cap\left\langle H_{i+1}\right\rangle_{H: G_{2}} \text { by }\left\langle H_{i}\right\rangle_{H: G_{3}}=\left\langle H_{i}\right\rangle_{H: G_{1}} \cap\left\langle H_{i}\right\rangle_{H: G_{2}}, S^{\prime \prime} \notin\left\langle H_{i+1}\right\rangle_{H: G_{1}} .
$$

The case of $S^{\prime} \notin\left\langle H_{i}\right\rangle_{H: G_{2}}, S^{\prime} \in\left\langle H_{i}\right\rangle_{H: G_{1}}$ is proved by a similar procedure and omitted.

Lemma 12. (i) Let $G^{\prime} \mid S^{\prime} \in \tau^{*}$ then $G_{G^{\prime} \mid S^{\prime}: S^{\prime}}^{*} \cap G_{G^{\prime} \mid S^{\prime}: G^{\prime}}^{*}=\varnothing, G_{G^{\prime} \mid S^{\prime}: G^{\prime}}^{*}\left|G_{G^{\prime} \mid S^{\prime}: S^{\prime}}^{*}=G\right| G^{*}$;

(ii) $H \in \tau^{*}, H^{\prime} \mid H^{\prime \prime} \subseteq H$ then $G_{H: H^{\prime} \mid H^{\prime \prime}}^{*}=G_{H: H^{\prime}}^{*} G_{H: H^{\prime \prime}}^{*}$.

Proof. (i) and (ii) are immediately from Lemma 11.

Notation 6. We write $\tau_{H_{i}^{c}: S_{11}^{c}}^{*}, G_{H_{i}^{c}: S_{i 1}^{c}}^{*}$ as $\tau_{S_{i 1}^{c}}^{*}, G_{S_{i 1}^{c}}^{*}$, respectively, for the sake of simplicity.

Lemma 13. (i) $G_{S_{i 1}^{c}}^{*} \subseteq G \mid G^{*}$;

(ii) $\tau_{S_{i 1}^{c}}^{*}$ is a derivation of $G_{S_{i 1}^{c}}^{*}$ from $S_{i 1}^{c}$, which we denote by $\frac{S_{i 1}^{c}}{G_{S_{i 1}^{c}}^{*}}\left\langle\tau_{S_{i 1}^{c}}^{*}\right|$;

(iii) $G_{S_{i u}^{c}}^{*}=S_{i u}^{c}$ and $\tau_{S_{i u}^{c}}^{*}$ consists of a single node $S_{i u}^{c}$ for all $2 \leqslant u \leqslant m_{i}$;

(iv) $v_{l}\left(G_{S_{i 1}^{c}}^{*}\right) \backslash v_{l}\left(S_{i 1}^{c}\right) \stackrel{i u}{=} v_{r}\left(G_{S_{i 1}^{c}}^{*}\right) \backslash v_{r}\left(S_{i 1}^{c}\right)$;

(v) $\langle H\rangle_{S_{i 1}^{c}} \in \tau_{S_{i 1}^{c}}^{*}$ implies $H \leqslant H_{i}^{c}$. Note that $\langle H\rangle_{S_{i 1}^{c}}$ is undefined for any $H>H_{i}^{c}$ or $H \| H_{i}^{c}$.

(vi) $S_{j}^{c} \in G_{S_{i 1}^{c}}^{*}$ implies $H_{i}^{c} \nless H_{j}^{c}$.

Proof. Claims from (i) to (v) follow immediately from Construction 1 and Lemma 4.

(vi) Since $S_{j}^{c} \in G_{S_{i 1}^{c}}^{*} \subseteq G \mid G^{*}$ then $S_{j}^{c}$ has the form $S_{j u}^{c}$ for some $u \geq 2$ by Notation 5 . Then $G_{S_{j}^{c}}^{*}=S_{j}^{c}$ by (iii). Suppose that $H_{i}^{c} \leqslant H_{j}^{c}$. Then $S_{j}^{c}$ is transferred from $H_{j}^{c}$ downward to $H_{i}^{c}$ and in side-hypersequent of $H_{i}^{c}$ by Notation 5 and $G \mid G^{*}<H_{i}^{c} \leqslant H_{j}^{c}$. Thus $\left\{S_{i 1}^{c}\right\} \cap\left\{S_{j}^{c}\right\}=\varnothing$ at $H_{i}^{c}$ since $S_{i 1}^{c}$ is the unique focus sequent of $H_{i}^{c}$. Hence $S_{j}^{c} \notin G_{S_{i 1}^{c}}^{*}$ by Lemma 11 and (iii), a contradiction therefore $H_{i}^{c} \nless H_{j}^{c}$.

Lemma 14. Let $\frac{G^{\prime}\left|S^{\prime} \quad G^{\prime \prime}\right| S^{\prime \prime}}{H \equiv G^{\prime}\left|G^{\prime \prime}\right| H^{\prime}}(I I) \in \tau^{*}$. (i) If $S_{j}^{c} \in G_{H: H^{\prime}}^{*}$ then $H_{j}^{c} \leqslant H$ or $H_{j}^{c} \| H$; (ii) If $S_{j}^{c} \in G_{H: G^{\prime \prime}}^{*}$ then $H_{j}^{c} \leqslant H$ or $H_{j}^{c} \| G^{\prime} \mid S^{\prime}$.

Proof. (i) We impose a restriction on (II) such that each sequent in $H^{\prime}$ is different from $S^{\prime}$ or $S^{\prime \prime}$ otherwise we treat it as an $(E W)$-application. Since $S_{j}^{c} \in G_{H: H^{\prime}}^{*} \subseteq G \mid G^{*}$ then $S_{j}^{c}$ has the form $S_{j u}^{c}$ for some $u \geq 2$ by Notation 5 . Thus $G_{S_{j}^{c}}^{*}=S_{j}^{c}$. Suppose that $H_{j}^{c}>H$. Then $S_{j}^{c}$ is transferred from $H_{j}^{c}$ downward to $H$. Thus $S_{j}^{c} \in H^{\prime}$ by $G_{S_{j}^{c}}^{\star}=S_{j}^{c} \in G_{H: H^{\prime}}^{*}$ and Lemma 11 . Hence $S_{j}^{c}=S^{\prime}$ or $S_{j}^{c}=S^{\prime \prime}$, a contradiction with the restriction above. Therefore $H_{j}^{c} \leqslant H$ or $H_{j}^{c} \| H$.

(ii) Let $S_{j}^{c} \in G_{H: G^{\prime \prime}}^{*}$. If $H_{j}^{c}>H$ then $S_{j}^{c} \in H$ by Proposition 2(i) and thus $S_{j}^{c} \in G^{\prime \prime}$ by Lemma 11 and, hence $H_{j}^{c} \| G^{\prime} \mid S^{\prime}$ by $H_{j}^{c} \geqslant G^{\prime \prime}\left|S^{\prime \prime}, G^{\prime}\right| S^{\prime} \| G^{\prime \prime} \mid S^{\prime \prime}$. If $H_{j}^{c} \| H$ then $H_{j}^{c} \| G^{\prime} \mid S^{\prime}$ by $H \leqslant G^{\prime} \mid S^{\prime}$. Thus $H_{j}^{c} \leqslant H$ or $H_{j}^{c} \| G^{\prime} \mid S^{\prime}$.

Definition 21. (i) By $H_{i}^{c} \leadsto H_{j}^{c}$ we mean that $S_{j u}^{c} \in G_{S_{i 1}^{c}}^{*}$ for some $2 \leqslant u \leqslant m_{j}$; (ii) By $H_{i}^{c} \leadsto H_{j}^{c}$ we mean that $H_{i}^{c} \leadsto H_{j}^{c}$ and $H_{j}^{c} \leadsto H_{i}^{c}$; (iii) $H_{i}^{c} \gg H_{j}^{c}$ means that $S_{j u}^{c} \notin G_{S_{i 1}^{c}}^{*}$ for all $2 \leqslant u \leqslant m_{j}$.

Then Lemma 13(vi) shows that $H_{i}^{c} \leadsto H_{j}^{c}$ implies $H_{i}^{c} \nless H_{j}^{c}$. 
Lemma 15. Let $H_{i}^{c} \| H_{j}^{c}, H_{i}^{c} \leadsto H_{j}^{c}, \frac{G^{\prime}\left|S^{\prime} G^{\prime \prime}\right| S^{\prime \prime}}{G^{\prime}\left|G^{\prime \prime}\right| H^{\prime}}(I I) \in \tau^{*}$ such that $G^{\prime}\left|S^{\prime} \leqslant H_{i}^{c}, G^{\prime \prime}\right| S^{\prime \prime} \leqslant H_{j}^{c}$. Then $S^{\prime} \in\left\langle G^{\prime} \mid S^{\prime}\right\rangle_{S_{i 1}^{c}}$.

Proof. Suppose that $S^{\prime} \notin\left\langle G^{\prime} \mid S^{\prime}\right\rangle_{S_{i 1}^{c}}$. Then $\left\langle G^{\prime} \mid S^{\prime}\right\rangle_{S_{i 1}^{c}} \subseteq G^{\prime}$ by $\left\langle G^{\prime} \mid S^{\prime}\right\rangle_{S_{i 1}^{c}} \subseteq G^{\prime} \mid S^{\prime},\left\langle G^{\prime}\left|G^{\prime \prime}\right| H^{\prime}\right\rangle_{S_{i 1}^{c}}=\left\langle G^{\prime} \mid S^{\prime}\right\rangle_{S_{i 1}^{c}}$ by Construction 1. Thus $\left\langle G^{\prime}\left|G^{\prime \prime}\right| H^{\prime}\right\rangle_{S_{i 1}^{c}} \subseteq G^{\prime}$. Hence $G^{\prime \prime} \mid H^{\prime} \cap\left\langle G^{\prime}\left|G^{\prime \prime}\right| H^{\prime}\right\rangle_{S_{i 1}^{c}}=\varnothing$ by Proposition 3(ii). Therefore $S_{j u}^{c} \notin G_{S_{i 1}^{c}}^{*}$ for all $1 \leqslant u \leqslant m_{j}$ by Lemma 11, i.e., $H_{i}^{c} \gg H_{j}^{c}$, a contradiction and hence $S^{\prime} \in\left\langle G^{\prime} \mid S^{\prime}\right\rangle_{S_{i 1}^{c}}$.

Lemma 13(ii) shows that $\tau_{S_{i 1}^{c}}^{*}$ is a derivation of $G_{S_{i 1}^{c}}^{*}$ from one premise $S_{i 1}^{c}$. We generalize it by introducing derivations from multiple premises in the following. In the remainder of this section, let $I=\left\{H_{i_{1}}^{c}, \cdots, H_{i_{m}}^{c}\right\} \subseteq\left\{H_{1}^{c}, \cdots, H_{N}^{c}\right\}, H_{i_{k}}^{c} \leadsto H_{i_{q}}^{c}$ for all $1 \leqslant k<q \leqslant m$. Then $H_{i_{k}}^{c} \nless H_{i_{q}}^{c}$ and $H_{i_{q}}^{c} \nless H_{i_{k}}^{c}$ by Lemma 13(vi) thus $H_{i_{k}}^{c} \| H_{i_{q}}^{c}$ for all $1 \leqslant k<q \leqslant m$.

Notation 7. $H_{I}^{V}$ denotes the intersection node of $H_{i_{1}}^{c}, \cdots, H_{i_{m}}^{c}$. We sometimes write the intersection node of $H_{i}^{c}$ and $H_{j}^{c}$ as $H_{i j}^{V}$. If $I=\left\{H_{i}^{c}\right\}, H_{I}^{V}:=H_{i}^{c}$, i.e., the intersection node of a single node is itself.

Let $\frac{G^{\prime}\left|S^{\prime} G^{\prime \prime}\right| S^{\prime \prime}}{G^{\prime}\left|G^{\prime \prime}\right| H^{\prime}}(I I) \in \tau^{*}$ such that $G^{\prime}\left|G^{\prime \prime}\right| H^{\prime}=H_{I}^{V}$. Then $I$ is divided into two subsets $I_{l}=\left\{H_{l_{1}}^{c}, \cdots, H_{l_{m(l)}}^{c}\right\}$ and $I_{r}=\left\{H_{r_{1}}^{c}, \cdots, H_{r_{m(r)}}^{c}\right\}$, which occur in the left subtree $\tau^{*}\left(G^{\prime} \mid S^{\prime}\right)$ and right subtree $\tau^{*}\left(G^{\prime \prime} \mid S^{\prime \prime}\right)$ of $\tau^{*}\left(G^{\prime}\left|G^{\prime \prime}\right| H^{\prime}\right)$, respectively.

Let $\mathcal{I}=\left\{S_{i_{1} 1}^{c}, \cdots, S_{i_{m} 1}^{c}\right\}, \mathcal{I}_{l}=\left\{S_{l_{1} 1}^{c}, \cdots, S_{l_{m(l)}}^{c}\right\}, \mathcal{I}_{r}=\left\{S_{r_{1} 1}^{c}, \cdots, S_{r_{m(r)}}^{c}\right\}$ such that $\mathcal{I}=\mathcal{I}_{l} \cup \mathcal{I}_{r}$. A derivation $\tau_{\mathcal{I}}^{*}$ of $\left\langle G \mid G^{*}\right\rangle_{\mathcal{I}}$ from $S_{i_{1} 1}^{c}, \cdots, S_{i_{m} 1}^{c}$ is constructed by induction on $|I|$. The base case of $|I|=1$ has been done by Construction 1. For the induction case, suppose that derivations $\tau_{\mathcal{I}_{l}}^{*}$ of $\left\langle G \mid G^{*}\right\rangle_{\mathcal{I}_{l}}$ from $S_{l_{1} 1}^{c}, \cdots, S_{l_{m(l)}}^{c}$ and $\tau_{\mathcal{I}_{r}}^{*}$ of $\left\langle G \mid G^{*}\right\rangle_{\mathcal{I}_{r}}$ from $S_{r_{1} 1}^{c}, \cdots, S_{r_{m(r)}}^{c}$ are constructed. Then $\tau_{\mathcal{I}}^{*}$ of $\left\langle G \mid G^{*}\right\rangle_{\mathcal{I}}$ from $S_{i_{1} 1}^{c}, \cdots, S_{i_{m} 1}^{c}$ is constructed as follows.

Construction 4. (Appendix A.5.2) (i)

$$
\begin{gathered}
\langle H\rangle_{\mathcal{I}}:=\langle H\rangle_{\mathcal{I}_{l}} \text { for all } G^{\prime} \mid S^{\prime} \leqslant H \leqslant H_{i}^{c} \text { for some } H_{i}^{c} \in I_{l}, \\
\langle H\rangle_{\mathcal{I}}:=\langle H\rangle_{\mathcal{I}_{r}} \text { for all } G^{\prime \prime} \mid S^{\prime \prime} \leqslant H \leqslant H_{i}^{c} \text { for some } H_{i}^{c} \in I_{r}, \\
\tau_{\mathcal{I}}^{*}\left(\left\langle G^{\prime} \mid S^{\prime}\right\rangle_{\mathcal{I}}\right):=\tau_{\mathcal{I}_{l}}^{*}\left(\left\langle G^{\prime} \mid S^{\prime}\right\rangle_{\mathcal{I}_{l}}\right), \tau_{\mathcal{I}}^{*}\left(\left\langle G^{\prime \prime} \mid S^{\prime \prime}\right\rangle_{\mathcal{I}}\right):=\tau_{\mathcal{I}_{r}}^{*}\left(\left\langle G^{\prime \prime} \mid S^{\prime \prime}\right\rangle_{\mathcal{I}_{r}}\right) ;
\end{gathered}
$$

(ii)

$$
\left\langle G^{\prime}\left|G^{\prime \prime}\right| H^{\prime}\right\rangle_{\mathcal{I}}:=\left\langle G^{\prime}\right\rangle_{\mathcal{I}_{l}}\left|\left\langle G^{\prime \prime}\right\rangle_{\mathcal{I}_{r}}\right| H^{\prime}
$$

and

$$
\frac{\left\langle G^{\prime} \mid S^{\prime}\right\rangle_{\mathcal{I}_{l}}\left\langle G^{\prime \prime} \mid S^{\prime \prime}\right\rangle_{\mathcal{I}_{r}}}{\left\langle G^{\prime}\left|G^{\prime \prime}\right| H^{\prime}\right\rangle_{\mathcal{I}}}(I I) \in \tau_{\mathcal{I}}^{*}
$$

(iii) Other nodes of $\tau_{\mathcal{I}}^{*}$ are built up by Construction 1(ii).

The following lemma is a generalization of Lemma 13.

Lemma 16. Let $\operatorname{Th}\left(H_{i_{k}}^{c}\right)=\left(H_{i_{k} 0}^{c}, \cdots, H_{i_{k} n_{i_{k}}}^{c}\right)$, where $1 \leqslant k \leqslant m, H_{i_{k} 0}^{c}=H_{i_{k}}^{c}$ and $H_{i_{k} n_{i_{k}}}^{c}=G \mid G^{*}$. Then, for all $0 \leqslant u \leqslant n_{i^{\prime}}$

(i)

$$
\left\langle H_{i_{k} u}^{c}\right\rangle_{\mathcal{I}}=\bigcap\left\{\left\langle H_{i_{k} u}^{c}\right\rangle_{S_{j 1}^{c}}: H_{j}^{c} \in I, H_{i_{k} u}^{c} \leqslant H_{j}^{c}\right\}
$$


(ii)

$$
\frac{\left\{S_{j 1}^{c}: H_{j}^{c} \in I, H_{i_{k} u}^{c} \leqslant H_{j}^{c}\right\}}{\left\langle H_{i_{k} u}^{c}\right\rangle_{\mathcal{I}}}\left\langle\tau_{\mathcal{I}}^{*}\left(\left\langle H_{i_{k} u}^{c}\right\rangle_{\mathcal{I}}\right)\right\rangle ;
$$

(iii)

$$
\begin{aligned}
& v_{l}\left(\left\langle H_{i_{k} u}^{c}\right\rangle_{\mathcal{I}}\right) \backslash \bigcup\left\{v_{l}\left(S_{j 1}^{c}\right): H_{j}^{c} \in I, H_{i_{k} u}^{c} \leqslant H_{j}^{c}\right\}= \\
& v_{r}\left(\left\langle H_{i_{k} u}^{c}\right\rangle_{\mathcal{I}}\right) \backslash \bigcup\left\{v_{r}\left(S_{j 1}^{c}\right): H_{j}^{c} \in I, H_{i_{k} u}^{c} \leqslant H_{j}^{c}\right\} ;
\end{aligned}
$$

(iv) $\langle H\rangle_{\mathcal{I}} \in \tau_{\mathcal{I}}^{*}$ if and only if $H \leqslant H_{i}^{c}$ for some $H_{i}^{c} \in I$. Note that $\langle H\rangle_{\mathcal{I}}$ is undefined if $H>H_{i}^{c}$ or $H \| H_{i}^{c}$ for all $H_{i}^{c} \in I$.

Proof. (i) is proved by induction on $|I|$. For the base step, let $|I|=1$ then the claim holds clearly. For the induction step, let $|I| \geqslant 2$ then $\left|I_{l}\right| \geqslant 1$ and $\left|I_{r}\right| \geqslant 1$. Then $S^{\prime} \in\left\langle G^{\prime} \mid S^{\prime}\right\rangle_{S_{i 1}^{c}}$ for all $H_{i}^{c} \in I_{l}$ by Lemma 15 and $H_{i}^{c} \leadsto H_{j}^{c}$ for all $H_{j}^{c} \in I_{r} .\left\langle G^{\prime} \mid S^{\prime}\right\rangle_{\mathcal{I}_{l}}=\bigcap_{H_{i}^{c} \in I_{l}}\left\langle G^{\prime} \mid S^{\prime}\right\rangle_{S_{i 1}^{c}}$ by the induction hypothesis then $S^{\prime} \in\left\langle G^{\prime} \mid S^{\prime}\right\rangle_{\mathcal{I}_{l}}$ thus $\left\langle G^{\prime}\left|G^{\prime \prime}\right| H^{\prime}\right\rangle_{\mathcal{I}_{l}}=\left\langle G^{\prime}\right\rangle_{\mathcal{I}_{l}}\left|G^{\prime \prime}\right| H^{\prime}$ by $G^{\prime} \mid S^{\prime} \leqslant H_{I_{l}}^{V}$.

$\left\langle G^{\prime}\left|G^{\prime \prime}\right| H^{\prime}\right\rangle_{\mathcal{I}_{r}}=\left\langle G^{\prime \prime}\right\rangle_{\mathcal{I}_{r}}\left|G^{\prime}\right| H^{\prime}$ holds by a procedure similar to above then

$$
\begin{aligned}
\left\langle G^{\prime}\left|G^{\prime \prime}\right| H^{\prime}\right\rangle_{\mathcal{I}} & =\left\langle G^{\prime}\right\rangle_{\mathcal{I}_{l}}\left|\left\langle G^{\prime \prime}\right\rangle_{\mathcal{I}_{r}}\right| H^{\prime} \\
& =\left(\left\langle G^{\prime}\right\rangle_{\mathcal{I}_{l}}\left|G^{\prime \prime}\right| H^{\prime}\right) \bigcap\left(\left\langle G^{\prime \prime}\right\rangle_{\mathcal{I}_{r}}\left|G^{\prime}\right| H^{\prime}\right) \\
& =\left\langle G^{\prime}\left|G^{\prime \prime}\right| H^{\prime}\right\rangle_{\mathcal{I}_{l}} \cap\left\langle G^{\prime}\left|G^{\prime \prime}\right| H^{\prime}\right\rangle_{\mathcal{I}_{r}}
\end{aligned}
$$

by $\left\langle G^{\prime}\right\rangle_{\mathcal{I}_{l}} \subseteq G^{\prime}$ and $\left\langle G^{\prime \prime}\right\rangle_{\mathcal{I}_{r}} \subseteq G^{\prime \prime}$. Other claims hold immediately from Construction 4 .

Lemma 17. (i) Let $G_{\mathcal{I}}^{*}$ denote $\left\langle G \mid G^{*}\right\rangle_{\mathcal{I}}$ then $G_{\mathcal{I}}^{*}=\bigcap_{H_{i}^{c} \in I} G_{S_{i 1}^{c}}^{*}$;

(ii) $\frac{S_{i_{1} 1}^{c} \cdots S_{i_{m} 1}^{c}}{G_{\mathcal{I}}^{*}}\left\langle\tau_{\mathcal{I}}^{*}\right\rangle$;

(iii) $v_{l}\left(G_{\mathcal{I}}^{*}\right) \backslash \bigcup_{H_{j}^{c} \in I} v_{l}\left(S_{j 1}^{c}\right)=v_{r}\left(G_{\mathcal{I}}^{*}\right) \backslash \bigcup_{H_{j}^{c} \in I} v_{r}\left(S_{j 1}^{c}\right)$;

(iv) $S_{j}^{c} \in G_{\mathcal{I}}^{*}$ implies $H_{i}^{c} \nless H_{j}^{c}$ for all $H_{i}^{c} \in I$.

Proof. (i), (ii) and (iii) follow immediately from Lemma 16. (iv) holds by (i) and Lemma 13 (vi).

Lemma 17 (iv) shows that there exists no copy of $S_{i_{k}}^{c}$ in $G_{\mathcal{I}}^{*}$ for any $1 \leqslant k \leqslant m$. Then we may regard them to be eliminated in $\tau_{\mathcal{I}}^{*}$. We then call $\tau_{\mathcal{I}}^{*}$ an elimination derivation.

Let $\mathcal{I}^{\prime}=\left\{S_{i_{1} u_{1}}^{c}, \cdots, S_{i_{m} u_{m}}^{c}\right\}$ be another set of sequents to $I$ such that $G^{\prime} \equiv S_{i_{1} u_{1}}^{c}|\cdots| S_{i_{m} u_{m}}^{c}$ is a copy of $G^{\prime \prime} \equiv S_{i_{1} 1}^{c}|\cdots| S_{i_{m}}^{c}$. Then $G^{\prime}$ and $G^{\prime \prime}$ are disjoint and there exist two bijections $\sigma_{l}: v_{l}\left(G^{\prime}\right) \rightarrow v_{l}\left(G^{\prime \prime}\right)$ and $\sigma_{r}: v_{r}\left(G^{\prime}\right) \rightarrow v_{r}\left(G^{\prime \prime}\right)$ such that $\sigma_{r} \circ \sigma_{l}\left(G^{\prime}\right)=G^{\prime \prime}$. By applying $\sigma_{r} \circ \sigma_{l}$ to $\tau_{\mathcal{I}}^{*}$, we construct a derivation from $S_{i_{1} u_{1}}^{c}, \cdots, S_{i_{m} u_{m}}^{c}$ and denote it by $\tau_{\mathcal{I}^{\prime}}^{*}$ and its root by $G_{\mathcal{I}^{\prime}}^{*}$.

Let $\mathbf{I}^{\prime}=\left\{G_{b_{1}}\left|S_{i_{1} u_{1}}^{c}, \cdots, G_{b_{m}}\right| S_{i_{m} u_{m}}^{c}\right\}$ be a set of hypersequents to $I$, where $G_{b_{k}} \mid S_{i_{k} u_{k}}^{c}$ be closed for all $1 \leqslant k \leqslant m$. By applying $\tau_{\mathcal{I}^{\prime}}^{*}$ to $S_{i_{1} u_{1}}^{c}, \cdots, S_{i_{m} u_{m}}^{c}$ in $G_{b_{1}}\left|S_{i_{1} u_{1}}^{c}, \cdots, G_{b_{m}}\right| S_{i_{m} u_{m}}^{c}$, we construct a derivation from

$$
G_{b_{1}}\left|S_{i_{1} u_{1}}^{c}, \cdots, G_{b_{m}}\right| S_{i_{m} u_{m}}^{c}
$$

and denote it by $\tau_{\mathbf{I}^{\prime}}^{*}$ and its root by $G_{\mathbf{I}^{\prime}}^{*}$. Then $G_{\mathbf{I}^{\prime}}^{*}=\left\{G_{b_{k}}\right\}_{k=1}^{m} \mid G_{\mathcal{I}^{\prime}}^{*}$.

Definition 22. We will use all $\tau_{\mathbf{I}^{\prime}}^{*}$ as rules of $\mathbf{G L}_{\Omega}$ and call them elimination rules. Further, we call $S_{i_{1} u_{1}}^{c}, \cdots, S_{i_{m} u_{m}}^{c}$ focus sequents and, all sequents in $G_{\mathcal{I}^{\prime}}^{*}$ principal sequents and, $G_{b_{1}}, \cdots, G_{b_{m}}$ side-hypersequents of $\tau_{\mathbf{I}^{\prime}}^{*}$ 
Remark 2. We regard Construction 1 as a procedure $\mathcal{F}$, whose inputs are $\tau^{2}, H, H^{\prime}$ and output $\tau_{H: H^{\prime}}^{2}$. With such a viewpoint, we write $\tau_{H: H^{\prime}}^{2}$ as $\mathcal{F}_{H: H^{\prime}}\left(\tau^{2}\right)$. Then $\tau_{\mathcal{I}}^{*}$ can be constructed by iteratively applying $\mathcal{F}$ to $\tau^{*}$, i.e., $\tau_{\mathcal{I}}^{*}=\mathcal{F}_{H_{i_{m}}^{c}: S_{i_{1} 1}^{c}}\left(\cdots \mathcal{F}_{H_{i_{1}}^{c}: S_{i_{1}}^{c}}\left(\tau^{*}\right) \cdots\right)$.

We replace locally each $\frac{G^{\prime}}{G^{\prime}}\left(I D_{\Omega}\right)$ in $\tau_{\mathcal{I}}^{*}$ with $G^{\prime}$ and denote the resulting derivation also by $\tau_{\mathcal{I}}^{*}$. Then each non-root node in $\tau_{\mathcal{I}}^{*}$ has the focus sequent.

Let $H \in \tau_{\mathcal{I}}^{*}$. Then there exists a unique node in $\tau^{*}$, which we denote by $\mathcal{O}(H)$ such that $H$ comes from $\mathcal{O}(H)$ by Constructions 1 and 4 . Then the focus sequent of $\mathcal{O}(H)$ in $\tau^{*}$ is the focus of $H$ in $\tau_{\mathcal{I}}^{*}$ if $H$ is a non-root node and, $\mathcal{O}(H)=H$ or $H \subseteq \mathcal{O}(H)$ as two hypersequents. Since the relative positions of any two nodes in $\tau^{*}$ are kept unchanged in constructing $\tau_{\mathcal{I}}^{*}, H_{1} \leqslant_{\tau_{\mathcal{I}}^{*}} H_{2}$ if and only if $\mathcal{O}\left(H_{1}\right) \leqslant \tau^{*} \mathcal{O}\left(H_{2}\right)$ for any $H_{1}, H_{2} \in \tau_{\mathcal{I}}^{*}$. Especially, $\mathcal{O}\left(S_{i_{k} 1}^{c}\right)=H_{i_{k}}^{c}$ for $S_{i_{k} 1}^{c} \in \tau_{\mathcal{I}}^{*}$.

Let $H \in \tau_{\mathcal{I}}^{*}$. Then $H^{\prime} \equiv \sigma_{r} \circ \sigma_{l}(H) \in \tau_{\mathcal{I}^{\prime}}^{*}$ and $H^{\prime \prime} \equiv\left\{G_{b_{k}}: H \leqslant_{\tau_{\mathcal{I}}^{*}} S_{i_{k} 1}^{c}\right.$ and $\left.1 \leqslant k \leqslant m\right\} \mid H^{\prime} \in \tau_{I^{\prime}}^{*}$. Define $\mathcal{O}\left(H^{\prime}\right)=\mathcal{O}\left(H^{\prime \prime}\right)=\mathcal{O}(H)$. Then $\mathcal{O}\left(G_{\mathbf{I}^{\prime}}^{*}\right)=G \mid G^{*}$ and $\mathcal{O}\left(G_{b_{k}} \mid S_{i_{k} u_{k}}^{c}\right)=H_{i_{k}}^{c}$ for all $G_{b_{k}} \mid S_{i_{k}}^{c} u_{k} \in \tau_{\mathbf{I}^{\prime}}^{*}$.

Since $G_{\mathcal{I}}^{*}=\left\langle G \mid G^{*}\right\rangle_{\mathcal{I}} \subseteq G \mid G^{*}$, then each $(p E C)$-sequent in $G_{\mathcal{I}}^{*}$ has the form $S_{j v}^{c}$ for some $1 \leqslant j \leqslant N$, $2 \leqslant v \leqslant m_{j}$ by Proposition 2(ii). Then we introduce the following definition.

Definition 23. (i) By $S_{j}^{c} \in G_{\mathcal{I}}^{*}$ we means that there exists $H \in \tau_{\mathcal{I}}^{*}$ such that $S_{j}^{c} \in H, \mathcal{O}(H)=H_{j}^{c}$. So is $S_{j}^{c} \in G_{\mathcal{I}^{\prime}}^{*}$.

(ii) Let $S_{j}^{c} \in G_{\mathcal{I}}^{*}$. By $H_{j}^{c} \leqslant \tau_{\mathcal{I}}^{*} H_{i}^{c}$ we means that there exist $H, H^{\prime} \in \tau_{\mathcal{I}}^{*}$ such that $S_{j}^{c} \in H, \mathcal{O}(H)=$ $H_{j}^{c}, \mathcal{O}\left(H^{\prime}\right)=H_{i}^{c}$ and $H_{j}^{c} \leqslant_{\tau^{*}} H_{i}^{c}$. We usually write $\leqslant_{\tau_{\mathcal{I}}^{*}}$ as $\leqslant$.

\section{Separation of One Branch}

In the remainder of this paper, we assume that $p$ occur at most one time for each sequent in $G_{0}$ as the one in the main theorem, $\tau$ be a cut-free proof of $G_{0}$ in GL and $\tau^{*}$ the proof of $G \mid G^{*}$ in $\mathbf{G L}_{\Omega}$ resulting from preprocessing of $\tau$. Then $\left|v_{l}(S)\right|+\left|v_{r}(S)\right| \leq 1$ for all $S \in G$, which plays a key role in discussing the separation of branches.

Definition 24. By $S^{\prime} \epsilon_{c} G^{\prime}$ we mean that there exists some copy of $S^{\prime}$ in $G^{\prime} . G^{\prime} \subseteq_{c} G^{\prime \prime}$ if $S^{\prime} \epsilon_{c} G^{\prime \prime}$ for all $S^{\prime} \in G^{\prime} . G^{\prime}=_{c} G^{\prime \prime}$ if $G^{\prime} \subseteq_{c} G^{\prime \prime}$ and $G^{\prime \prime} \subseteq_{c} G^{\prime}$. Let $G_{11}, \cdots, G_{1 m}$ be $m$ copies of $G_{1}$ then we denote $G^{\prime}\left|G_{11}\right| \cdots \mid G_{1 m}$ by $G^{\prime} \mid\left\{G_{1 u}\right\}_{u=1}^{m}$ or $G^{\prime} \mid\left\{G_{1}\right\}^{m}$.

Definition 25. Let $I=\left\{H_{i_{1}}^{c}, \cdots, H_{i_{m}}^{c}\right\} \subseteq\left\{H_{1}^{c}, \cdots, H_{N}^{c}\right\}, H_{i_{k}}^{c} \| H_{i_{l}}^{c}$ for all $1 \leqslant k<l \leqslant m$. $\left\lceil S_{i_{k}}^{c}\right]_{I}$ is called a branch of $H_{i_{k}}^{c}$ to I if it is a closed hypersequent such that

(i) $\left\lceil S_{i_{k}}^{c}\right\rceil_{I} \subseteq_{c} G \mid G^{*}$,

(ii) $S_{i_{k}}^{c} \in\left[S_{i_{k}}^{c}\right]_{I}$,

(iii) $S_{j}^{c} \in\left\lceil S_{i_{k}}^{c}\right\rceil_{I}$ implies $H_{j}^{c} \leqslant H_{i_{k}}^{c}$ or $H_{j}^{c} \| H_{i}^{c}$ for all $H_{i}^{c} \in I$.

Then (i) $S_{i_{l}}^{c} \notin_{c}\left\lceil S_{i_{k}}^{c}\right]_{I}$ for all $1 \leqslant k, l \leqslant m, k \neq l$; (ii) $S_{j}^{c} \in\left\lceil S_{i_{k}}^{c}\right]_{I}$ and $H_{j}^{c} \nless H_{i_{k}}^{c}$ imply $H_{j}^{c} \notin I$.

In this section, let $I=\left\{H_{i}^{c}\right\}, \mathbf{I}=\left\{\left[S_{i}^{c}\right\rceil_{I}\right\}$, we will give an algorithm to eliminate all $S_{j}^{c} \in\left\lceil S_{i}^{c}\right]_{I}$ satisfying $H_{j}^{c} \leqslant H_{i}^{c}$.

Construction 5. (Appendix A.3) A sequence of hypersequents $G_{\mathbf{I}}^{\hat{i j}(q)}$ and their derivations $\tau_{\mathbf{I}}^{\hat{j}(q)}$ from $\left\lceil S_{i}^{c}\right]_{I}$ for all $q \geqslant 0$ are constructed inductively as follows.

For the base case, define $G_{I}^{i(0)}$ to be $\left\lceil S_{i}^{c}\right\rceil_{I}$ and, $\tau_{I}^{i j(0)}$ be $\overline{\overline{G_{I}^{i j(0)}}}$. For the induction case, suppose that $\tau_{\mathbf{I}}^{i(q)}$ and $G_{\mathbf{I}}^{i(q)}$ are constructed for some $0 \leqslant q$. If there exists no $S_{j}^{c} \in G_{\mathbf{I}}^{i(q)}$ such that $H_{j}^{c} \leqslant H_{i}^{c}$, then the procedure terminates and define $J_{\mathbf{I}}$ to be q; otherwise define $H_{i_{q}}^{c}$ such that $S_{i_{q}}^{c} \in G_{\mathbf{I}}^{\text {in }(q)}, H_{i_{q}}^{c} \leqslant H_{i}^{c}$ and $H_{j}^{c} \leqslant H_{i_{q}}^{c}$ for all $S_{j}^{c} \in G_{\mathbf{I}}^{i(q)}, H_{j}^{c} \leqslant H_{i}^{c}$. Let $S_{i_{q} 1}^{c}, \cdots, S_{i_{q} m_{q}}^{c}$ be all copies of $S_{i_{q}}^{c}$ in $G_{\mathbf{I}}^{\mathfrak{i}(q)}$ then define 
$G_{\mathbf{I}}^{i j(q+1)}=G_{\mathbf{I}}^{i(q)} \backslash\left\{S_{i_{q} u}^{c}\right\}_{u=1}^{m_{q}} \mid\left\{G_{S_{i q u}^{c}}^{*}\right\}_{u=1}^{m_{q}}$ and its derivation $\tau_{\mathbf{I}}^{\hbar(q+1)}$ is constructed by sequentially applying $\tau_{S_{i q 1}^{c}}^{*}, \cdots, \tau_{S_{i_{q} m_{q}}^{c}}^{*}$ to $S_{i_{q}}^{c}, \cdots, S_{i_{q} m_{q}}^{c}$ in $G_{I}^{\hbar(\tau)}$, respectively. Notice that we assign new identification numbers to new occurrences of $p$ in $\tau_{S_{i_{q u}}^{c}}^{*}$ for all $0 \leqslant q \leqslant J_{\mathbf{I}}-1,1 \leqslant u \leqslant m_{q}$.

Lemma 18. (i) $H_{i_{0}}^{c}=H_{i}^{c}$ and $H_{i_{q+1}}^{c}<H_{i_{q}}^{c}$ for all $0 \leqslant q \leqslant J_{\mathbf{I}}-2$;

(ii) $G_{\mathbf{I}}^{\hat{i}(q)} \subseteq_{c} G \mid G^{*}$ is closed for all $0 \leqslant q \leqslant J_{\mathbf{I}}$;

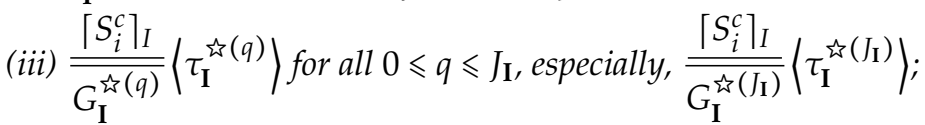

(iv) $S_{j}^{c} \in G_{\mathbf{I}}^{i j\left(J_{\mathbf{I}}\right)}$ implies $H_{j}^{c} \| H_{i}^{c}$ and,$S_{j}^{c} \in G_{S_{i q u}^{c}}^{*}$ for some $\tau_{G_{b} \mid S_{i q u}^{c}}^{*} \in \tau_{\mathbf{I}}^{i j\left(J_{\mathbf{I}}\right)}$ or $S_{j}^{c} \in\left\lceil S_{i}^{c}\right\rceil_{I}, H_{j}^{c} \nless H_{i}^{c}$, where $G_{b}=G_{I}^{\mathfrak{i}(q)} \backslash\left\{S_{i_{q} v}^{c}\right\}_{v=1}^{u}\left|\left\{G_{S_{i q v}^{c}}^{*}\right\}_{v=1}^{u-1}, G_{b}\right| S_{i_{q} u}^{c}$ is closed and $0 \leqslant q \leqslant J_{I}-1,1 \leqslant u \leqslant m_{q}$.

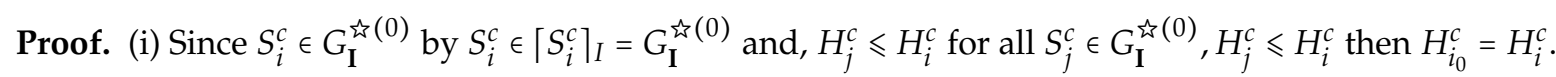
If $S_{i_{q+1}}^{c} \in G_{\mathbf{I}}^{i(q)} \backslash\left\{S_{i_{q} u}^{c}\right\}_{u=1}^{m_{q}}$ then $H_{i_{q+1}}^{c} \leqslant H_{i_{q}}^{c}$ by $S_{i_{q+1}}^{c} \in G_{\mathbf{I}}^{i n(q)}, H_{i_{q+1}}^{c} \leqslant H_{i}^{c}$ thus $H_{i_{q+1}}^{c}<H_{i_{q}}^{c}$ by all copies of $S_{i_{q}}^{c}$ in $G_{I}^{i j(q)}$ being collected in $\left\{S_{i_{q} u}^{c}\right\}_{u=1}^{m_{q}}$. If $S_{i_{q+1}}^{c} \in\left\{G_{S_{i_{q} u}^{c}}^{*}\right\}_{u=1}^{m_{q}}$ then $H_{i_{q}}^{c} \nless H_{i_{q+1}}^{c}$ by Lemma 13(vi) thus $H_{i_{q+1}}^{c}<H_{i_{q}}^{c}$ by $H_{i_{q}}^{c} \leqslant H_{i}^{c}, H_{i_{q+1}}^{c} \leqslant H_{i}^{c}$. Then $H_{i_{q+1}}^{c}<H_{i_{q}}^{c}$ by $G_{I}^{i j(q+1)}=G_{I}^{i j}(q) \backslash\left\{S_{i_{q} u}^{c}\right\}_{u=1}^{m_{q}} \mid\left\{G_{S_{i_{q} u}^{c}}^{*}\right\}_{u=1}^{m_{q}}$. Note that $H_{i_{I_{\mathrm{I}}}^{c}}^{c}$ is undefined in Construction 5 .

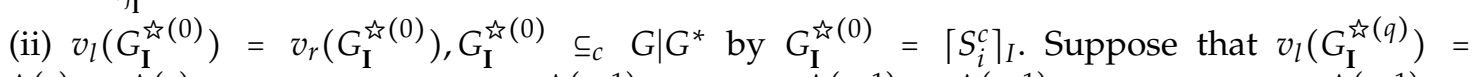

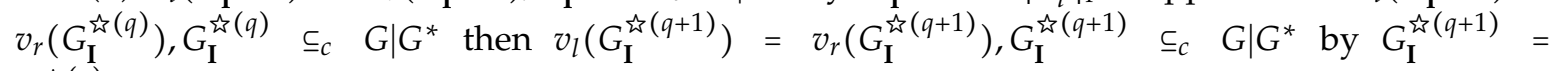
$G_{\mathbf{I}}^{i \tau}(q) \backslash\left\{S_{i_{q} u}^{c}\right\}_{u=1}^{m_{q}} \mid\left\{G_{S_{i q u}^{c}}^{*}\right\}_{u=1}^{m_{q}}, v_{l}\left(G_{S_{i q u}^{c}}^{*} \backslash\left\{S_{i_{q} u}^{c}\right\}\right)=v_{r}\left(G_{S_{i q u}^{c}}^{*} \backslash\left\{S_{i_{q} u}^{c}\right\}\right)$ and $G_{S_{i q u}^{c}}^{*} \subseteq_{c} G \mid G^{*}$ for all $1 \leqslant u \leqslant m_{q}$.

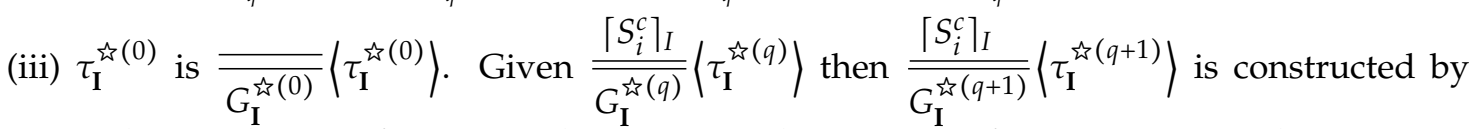
linking up the conclusion of previous derivation to the premise of its successor in the sequence of derivations

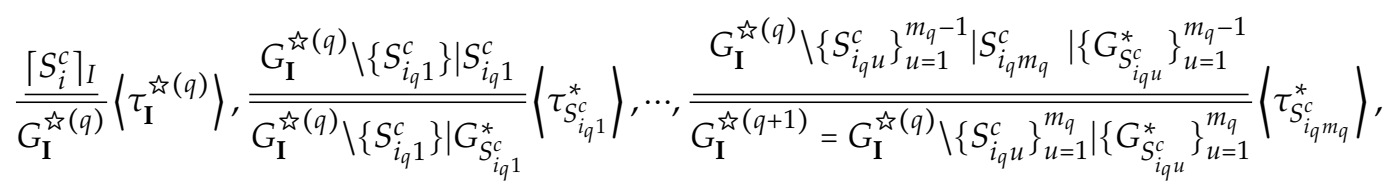

as shown in Figure 14.

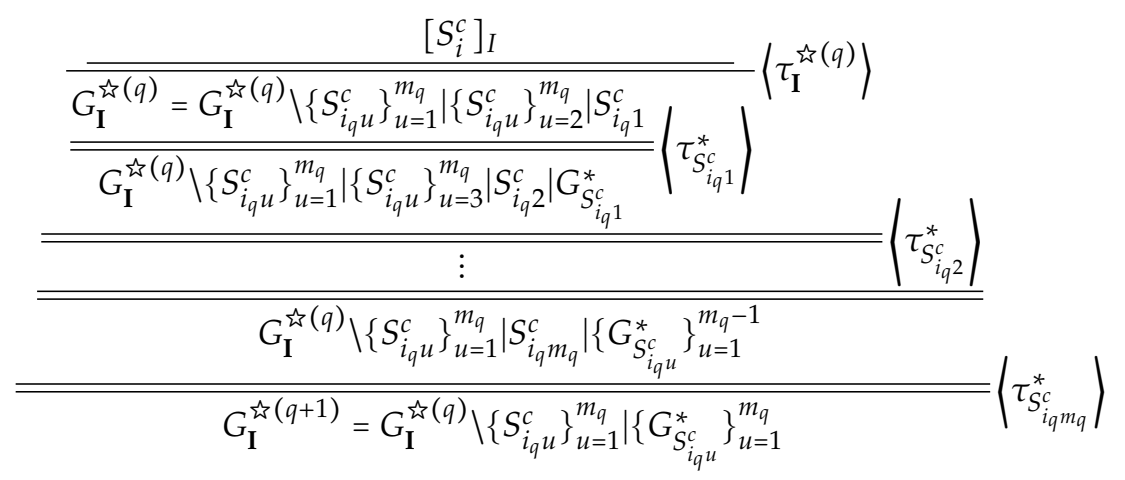

Figure 14. A derivation of $G_{I}^{\hbar(q+1)}$ from $G_{I}^{i(q)}$. 
(iv) Let $S_{j}^{c} \in G_{\mathbf{I}}^{\mathcal{h}^{2}\left(J_{\mathbf{I}}\right)}$. Then $H_{j}^{c} \nless H_{i}^{c}$ by the definition of $J_{\mathbf{I}}$. If $S_{j}^{c} \in\left\lceil S_{i}^{c}\right\rceil_{I}$, then $H_{j}^{c} \| H_{i}^{c}$ by $H_{j}^{c} \nless H_{i}^{c}$ and the definition of $\left[S_{i}^{c}\right]_{I}$. Otherwise, by Construction 5, there exists some $\tau_{G_{b} \mid S_{i q u}^{c}}^{*}$ in $\tau_{\mathbf{I}}^{\hat{I}^{2}\left(J_{\mathbf{I}}\right)}$ such that $S_{j}^{c} \in G_{S_{i_{q}}^{c} u}^{*}$. Then $H_{i_{q}}^{c} \nless H_{j}^{c}$ by Lemma 13(vi). Thus $H_{i}^{c} \nless H_{j}^{c}$ by $H_{i_{q}}^{c} \leqslant H_{i}^{c}$. Hence $H_{j}^{c} \| H_{i}^{c}$.

Lemma 18 shows that Construction 5 presents a derivation $\tau_{\mathbf{I}}^{\mathcal{\lambda}\left(J_{\mathbf{I}}\right)}$ of $G_{\mathbf{I}}^{\text {is }\left(J_{\mathbf{I}}\right)}$ from $\left\lceil S_{i}^{c}\right\rceil_{I}$ such that there does not exist $S_{j}^{c} \in G_{\mathbf{I}}^{\mathcal{i}\left(J_{\mathrm{I}}\right)}$ satisfying $H_{j}^{c} \leqslant H_{i}^{c}$, i.e., all $S_{j}^{c} \in\left\lceil S_{i}^{c}\right\rceil_{I}$ satisfying $H_{j}^{c} \leqslant H_{i}^{c}$ are eliminated by Construction 5 . We generalize this procedure as follows.

Construction 6. Let $H \in \tau^{*}, H_{1} \subseteq H$ and $H_{2} \subseteq_{c} G \mid G^{*}$. Then $G_{H: H_{l}}^{i\left(J_{H} H_{l}\right)}$ and its derivation $\tau_{H: H_{l}}^{i \sim\left(H_{H}\right)}$ for $l=1,2$

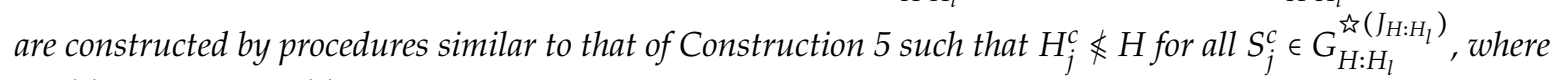

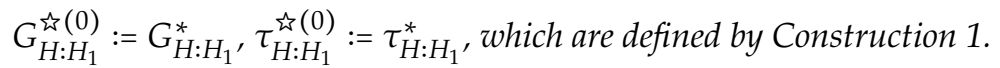

We sometimes write $J_{\mathbf{I}}, J_{H: H_{l}}$ as $J$ for simplicity. Then the following lemma holds clearly.

Lemma 19. (i) $\frac{H_{l}}{G_{H: H_{l}}^{\grave{i}(J)}}\left\langle\tau_{H: H_{l}}^{i(J)}\right\rangle, H_{j}^{c} \nless H$ for all $S_{j}^{c} \in G_{H: H_{l}}^{\grave{2}(J)}$.

(ii) If $S_{i}^{c} \in H$ and $H_{i}^{c}>H$ then $G_{H: S_{i}^{c}}^{i(J)}=S_{i}^{c}$.

(iii) If $S \epsilon_{c} G$ or, $S \epsilon_{c} G^{*}$ is a copy of $S_{i 1}^{c}$ and $H_{i}^{c} \nless H$ then $G_{H: S}^{i s(J)}=S$.

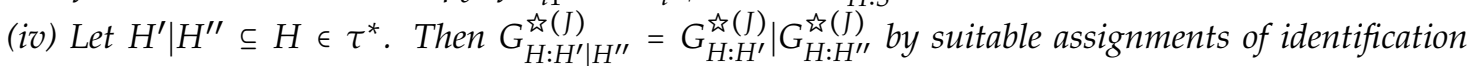
numbers to new occurrences of $p$ in constructing $\tau_{H: H^{\prime} \mid H^{\prime \prime}}^{\text {in }(J)} \tau_{H: H^{\prime}}^{i \text { ind }\left(H^{\prime \prime}\right.}$ and $\tau_{H: H^{\prime \prime}}^{i(J)}$.

(v) $G_{\mathrm{I}}^{i \mathcal{\sim}(J)}=\bigcup\left\{G_{H_{i}^{c}: S_{j}^{c}}^{i(J)}: S_{j}^{c} \in\left\lceil S_{i}^{c}\right\rceil_{I}, H_{j}^{c} \leqslant H_{i}^{c}\right\}\left|\cup\left\{S_{j}^{c}: S_{j}^{c} \in\left\lceil S_{i}^{c}\right\rceil_{I}, H_{j}^{c} \nless H_{i}^{c}\right\}\right| \cup\left\{S: S \in\left\lceil S_{i}^{c}\right\rceil_{I}, S \in_{c} G\right\}$.

Proof. Part (i) is proved by a procedure similar to that of Lemma 18(iii) and (iv), and omitted.

(ii) Since $S_{i 1}^{c}$ is the focus sequent of $H_{i}^{c}$ then it is revised by some rule at the node lower than $H_{i}^{c}$. Thus $S_{i}^{c} \in H$ is some copy of $S_{i 1}^{c}$ by $H_{i}^{c}>H$. Hence $S_{i}^{c}$ has the form $S_{i u}^{c}$ for some $u \geq 2$. Therefore it is transferred downward to $G \mid G^{*}$, i.e., $S_{i}^{c} \in G \mid G^{*}$. Then $G_{H: S_{i}^{c}}^{i(0)}=G_{H: S_{i}^{c}}^{*}=S_{i}^{c}$. Since there exists no $S_{j}^{c} \in G_{H: S_{j}^{c}}^{i j(0)}, H_{j}^{c} \leqslant H$ then $J=0$. Thus $G_{H: S_{i}^{c}}^{i j(J)}=S_{i}^{c}$.

(iii) is proved by a procedure similar to that of (ii) and omitted.

(iv) Since $H^{\prime} \mid H^{\prime \prime} \subseteq H \in \tau^{*}$, then $H^{\prime} \cap H^{\prime \prime}=\varnothing$ by Proposition 3. Thus $G_{H: H^{\prime} \mid H^{\prime \prime}}^{\mathfrak{i}(0)}=G_{H: H^{\prime} \mid H^{\prime \prime}}^{*}=$

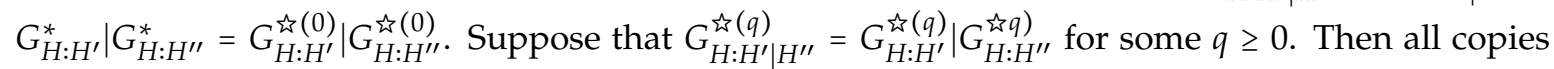
$\left\{S_{i_{q} u}^{c}\right\}_{u=1}^{m_{q}}$ of $S_{i_{q}}^{c}$ in $G_{H: H^{\prime} \mid H^{\prime \prime}}^{i(q)}$ are divided two subsets $\left\{S_{i_{q} u}^{c}\right\}_{u=1}^{m_{q}} \cap G_{H: H^{\prime}}^{\mathfrak{i}(q)}$ and $\left\{S_{i_{q} u}^{c}\right\}_{u=1}^{m_{q}} \cap G_{H: H^{\prime \prime}}^{\mathfrak{i}(q)}$. Thus we

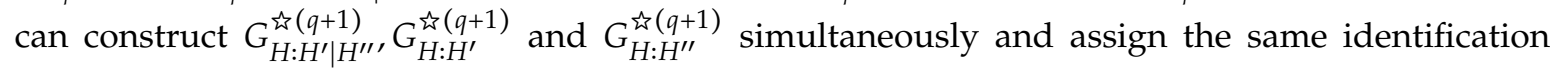
numbers to new occurrences of $p$ in $G_{H: H^{\prime}}^{i \text { in }(q+1)}$ and $G_{H: H^{\prime \prime}}^{\text {in }(q+1)}$ as the corresponding one in $G_{H: H^{\prime} \mid H^{\prime \prime}}^{\text {is }(q+1)}$.

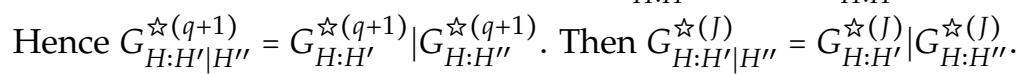

Note that the requirement is imposed only on one derivation that distinct occurrence of $p$ has

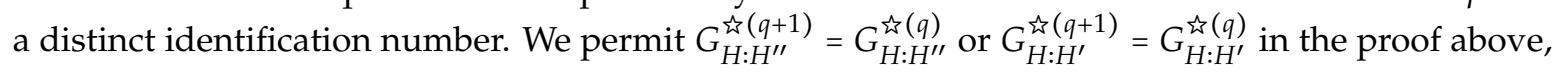
which has no essential effect on the proof of the claim.

(v) is immediately from (iv).

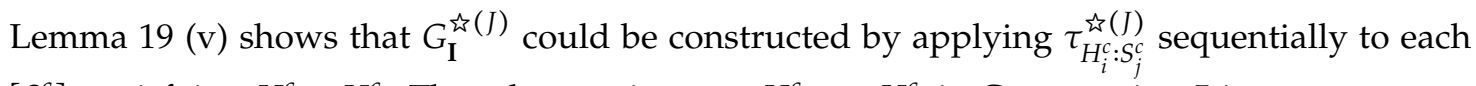
$S_{j}^{c} \in\left[S_{i}^{c}\right\rceil_{I}$ satisfying $H_{j}^{c} \leqslant H_{i}^{c}$. Thus the requirement $H_{i_{q+1}}^{c}<H_{i_{q}}^{c}$ in Construction 5 is not necessary, but which make the termination of the procedure obvious. 
Construction 7. Apply $\left(E C_{\Omega}^{*}\right)$ to $G_{I}^{\text {is }(J)}$ and denote the resulting hypersequent by $G_{I}^{\text {is }}$ and its derivation by $\tau_{\mathbf{I}}^{\text {in }}$. It is possible that $\left(E C_{\Omega}^{*}\right)$ is not applicable to $G_{\mathbf{I}}^{\hat{i}(J)}$ in which case we apply $\left\langle I D_{\Omega}\right\rangle$ to it for the regularity of the derivation.

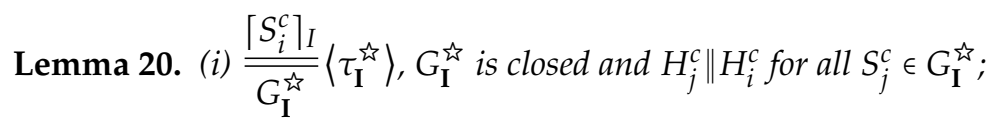

(ii) $\tau_{\mathbf{I}}^{\text {光 }}$ is constructed by applying elimination rules, say, $\frac{G_{b} \mid S_{i_{q} u}^{c}}{\overline{G_{b} \mid G_{S_{i q u}^{c}}^{*}}}\left|\tau_{G_{b} \mid S_{i_{q} u}^{c}}^{*}\right|$, and the fully constraint contraction rules, say, $\frac{G_{2}}{\bar{G}_{1}}\left\langle E C_{\Omega}^{*}\right\rangle$, where $H_{i_{q}}^{c} \leqslant H_{i}^{c}, G_{b} \mid S_{i_{q} u}^{c}$ is closed for $0 \leqslant q \leqslant J-1,1 \leqslant u \leqslant m_{q}$.

Proof. The proof follows immediately from Lemma 18.

Definition 26. Let $G^{\prime} \in G_{\mathbf{I}}^{\hat{\jmath}(J)}, H^{\prime} \subseteq G^{\prime}$ and $S^{\prime} \in H^{\prime}$.

(i) For any sequent-formula $A$ of $S^{\prime}$, define $\widehat{A}$ to be the sequent $S$ of $G_{\mathbf{I}}^{\text {is }(J)}$ such that $A$ is a sequent-formula of $S$ or subformula of a sequent-formula of $S$.

(ii) Let $S^{\prime}$ be in the form $A_{1}, \cdots, A_{n} \Rightarrow B_{1}, \cdots, B_{m}$, define $\widehat{S^{\prime}}$ to be the hypersequent which consists of all distinct sequents among $\widehat{A_{1}}, \cdots, \widehat{A_{n}}, \widehat{B_{1}}, \cdots, \widehat{B_{m}}$; (iii) Let $H^{\prime}$ be in the form $S_{1}|\cdots| S_{m}$, define $\widehat{H^{\prime}}$ to be $\widehat{S_{1}}|\cdots| \widehat{S_{m}}$.

(iv) We call $H^{\prime}$ to be separable if $\widehat{H^{\prime}} \subseteq_{c} G$ and, call it to be separated into $\widehat{H^{\prime}}$.

Note that $\tau_{\mathbf{I}}^{\mathcal{2}(J)}$ is a derivation without $\left(E C_{\Omega}\right)$ in $\mathbf{G L}_{\Omega}$. Then we can extract elimination derivations from it by Construction 1 .

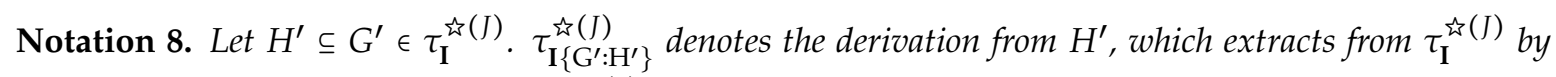
Construction 1 , and denote its root by $G_{\mathbf{I}\left\{G^{\prime}: H^{\prime}\right\}}^{\beth(J)}$.

The following two lemmas show that Constructions 5 and 6 force some sequents in $\left\lceil S_{i}^{c}\right\rceil_{I}$ or $H^{\prime}$ to be separable.

Lemma 21. Let $\frac{G^{\prime}\left|S^{\prime} \quad G^{\prime \prime}\right| S^{\prime \prime}}{H \equiv G^{\prime}\left|G^{\prime \prime}\right| H^{\prime}}(I I) \in \tau^{*}$. Then

(i) $H^{\prime}$ is separable in $\tau_{\mathrm{H}: \mathrm{H}^{\prime}}^{\mathfrak{i}^{\prime}(J)}$.

(ii) If $\frac{G_{b}\left|\left\langle G^{\prime} \mid S^{\prime}\right\rangle_{S_{q_{q} u}^{c}} G^{\prime \prime}\right| S^{\prime \prime}}{H_{1} \equiv G_{b}\left|\left\langle G^{\prime}\right\rangle_{S_{i q u}^{c}}\right| G^{\prime \prime} \mid H^{\prime}}(I I) \in \tau_{G_{b} \mid S_{i q u}^{c}}^{*} \in \tau_{\mathbf{I}}^{\text {出}}$, then $H^{\prime}$ is separable in $\tau_{\mathbf{I}}^{i(J)}$ and there is a unique copy of $\widehat{S^{\prime \prime}} \mid G_{\mathbf{I}\left\{H_{1}: G^{\prime \prime}\right\}}^{\text {in }(J)} G_{\mathbf{I}}^{\text {is }}$.

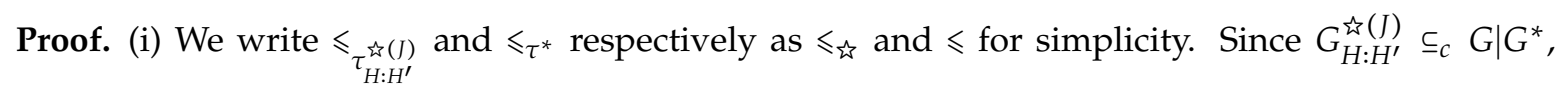
we divide it into two hypersequents $G_{H: H^{\prime}}^{0(J)}$ and $G_{H: H^{\prime}}^{*(J)}$ such that $G_{H: H^{\prime}}^{\hbar(J)}=G_{H: H^{\prime}}^{O(J)} \mid G_{H: H^{\prime}}^{*(J)} G_{H: H^{\prime}}^{0(J)} \subseteq_{c} G$, $G_{H: H^{\prime}}^{*(J)} \subseteq_{c} G^{*}$.

Let $S_{j}^{c} \in G_{H: H^{\prime}}^{*(J)}$ then $H_{j}^{c} \nless H$ by Construction 6. We prove that $H_{j}^{c} \| H^{\prime}$ in $\tau_{H: H^{\prime}}^{i(J)}$ as follows. If $S_{j}^{c} \in G_{H: H^{\prime}}^{*}$ then $H_{j}^{c} \| H^{\prime}$ in $\tau_{H: H^{\prime}}^{*}$ by Lemma 14(i), $\tau_{H: H^{\prime}}^{*} \in \tau_{H: H^{\prime}}^{\hbar(J)}$ and $H_{j}^{c} \nless H$. Thus we assume that $S_{j}^{c} \notin G_{H: H^{\prime}}^{*}$ in the following.

Then, by Lemma 18(iv), there exists some $\tau_{G_{b} \mid S_{i}^{c}}^{*}$ in $\tau_{H: H^{\prime}}^{i j(J)}$ such that $H_{i}^{c} \leqslant H, S_{j}^{c} \in G_{S_{i}^{c}}^{*}$. Then $H_{i}^{c} \nless H_{j}^{c}$ by Lemma 13 (vi). $H_{j}^{c} \nless H_{i}^{c}$ by $H_{i}^{c} \leqslant H, H_{j}^{c} \nless H$. Thus $H_{i}^{c} \|_{\tau^{*}} H_{j}^{c}$. Let $\frac{G_{1}\left|S_{1} \quad G_{2}\right| S_{2}}{G_{1}\left|G_{2}\right| H_{2}}(I I) \in \tau^{*}$, where 
$G_{1}\left|G_{2}\right| H_{2}=H_{i j}^{V}, G_{1}\left|S_{1} \leqslant H_{i}^{c}, G_{2}\right| S_{2} \leqslant H_{j}^{c}$. Then $S_{1} \in\left\langle G_{1} \mid S_{1}\right\rangle_{S_{i}^{c}}, G_{b}\left|\left\langle G_{1} \mid S_{1}\right\rangle_{S_{i}^{c}} \in \tau_{H: H^{\prime}}^{\hbar \vec{n}(J)}, G_{2}\right| S_{2} \in \tau_{H: H^{\prime}}^{\hbar \vec{n}(J)}$

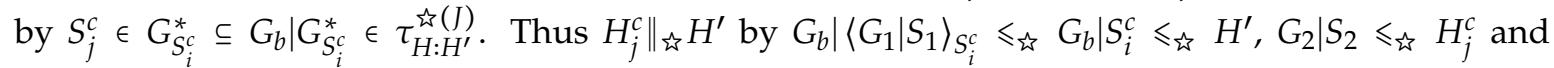
$G_{b}\left|\left\langle G_{1} \mid S_{1}\right\rangle_{S_{i}^{c}} \|_{\text {is }} G_{2}\right| S_{2}$.

Thus $H_{j}^{c} \| H^{\prime}$ in $\tau_{H: H^{\prime}}^{\imath(J)}$. Therefore $G_{H: H^{\prime}}^{*(J)} \cap \widehat{H^{\prime}}=\varnothing$. Then $\widehat{H^{\prime}} \subseteq G_{H: H^{\prime}}^{0(J)} \subseteq_{c} G$, i.e., $H^{\prime}$ is separable in $\tau_{\mathrm{H}: \mathrm{H}^{\prime}}^{i(J)}$.

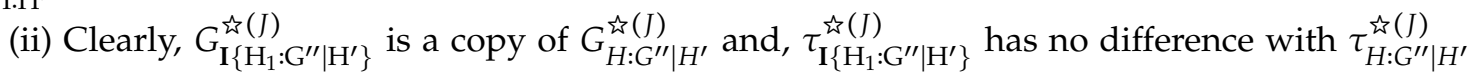
except some applications of $\left(I D_{\Omega}\right)$ and identification numbers of some $p^{\prime} s$. Then $H^{\prime}$ is separated into $\widehat{H^{\prime}}$ in $G_{\mathbf{I}\left\{H_{1}: H^{\prime}\right\}}^{\not \zeta()}$ by the same reason as that of (i). Then $S^{\prime}, S^{\prime \prime}$ are separated into $\widehat{S^{\prime}}$ and $\widehat{S^{\prime \prime}}$ in $\tau_{\mathbf{I}}^{\varkappa(J)}$, respectively. Then $\widehat{S^{\prime \prime}}\left|G_{\mathbf{I}\left\{H_{1}: G^{\prime \prime}\right\}}^{\varkappa^{2}(J)} \subseteq G_{\mathbf{I}}^{0(J)}\right| G_{\mathbf{I}}^{*(J)}$ is closed since $G^{\prime \prime} \mid S^{\prime \prime}$ is closed. Thus all copies of $\widehat{S^{\prime \prime}} \mid G_{\mathbf{I}\left\{H_{1}: G^{\prime \prime}\right\}}^{i n}$ in $\tau_{\mathbf{I}}^{i(J)}$ are contracted into one by $\left(E C_{\Omega}^{*}\right)$ in $G_{\mathbf{I}}^{i j}$.

Lemma 22. (i) All copies of $S_{i}^{c}$ in $\left\lceil S_{i}^{c}\right\rceil_{I}$ are separable in $\tau_{\mathbf{I}}^{\text {虫(J) }}$.

(ii) Let $H \in \tau^{*}, H^{\prime} \subseteq H, H_{j}^{c} \leqslant H$ or $H_{j}^{c} \| H$ for all $S_{j}^{c} \in G_{H: H^{\prime}}^{*}$. Then $H^{\prime}$ is separable in $\tau_{H: H^{\prime}}^{\hat{i}(J)}$.

Proof. Parts (i) and (ii) are proved by a procedure similar to that of Lemma 21 and are omitted.

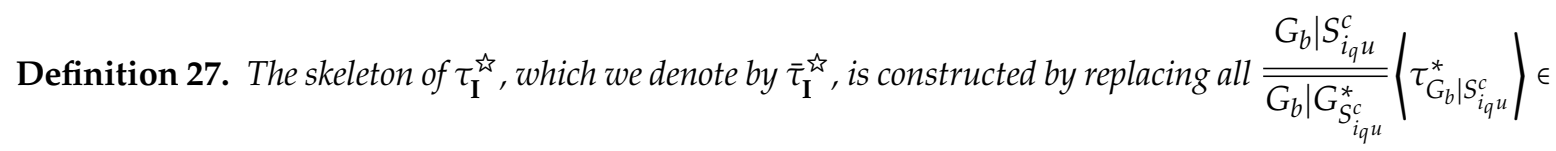
$\tau_{\mathbf{I}}^{\text {is }}$ with $\frac{G_{b} \mid S_{i_{q} u}^{c}}{G_{b} \mid G_{S_{i_{q} u}^{c}}^{*}}\left(\tau_{G_{b} \mid S_{i q u}^{c}}^{*}\right)$, i.e, $G_{b} \mid S_{i_{q} u}^{c}$ is the parent node of $G_{b} \mid G_{S_{i q u}^{c}}^{*}$ in $\bar{\tau}_{\mathbf{I}}^{i s}$.

Lemma 23. The parameter $\bar{\tau}_{\mathbf{I}}^{\bar{T}^{2}}$ is a linear structure with the lowest node $G_{\mathbf{I}}^{\text {is }}$ and the highest $\left\lceil S_{i}^{c}\right\rceil_{I}$.

Proof. It holds by all $\tau_{G_{b} \mid S_{i_{q} u}^{c}}^{*}$ and $E C_{\Omega}^{*}$ in $\tau_{\mathbf{I}}^{\text {光 }}$ being one-premise rules.

Definition 28. We call Construction 5 together with 7 the separation algorithm of one branch and, Construction 6 the separation algorithm along $H$.

\section{Separation Algorithm of Multiple Branches}

In this section, let $I=\left\{H_{i_{1}}^{c}, \cdots, H_{i_{m}}^{c}\right\} \subseteq\left\{H_{1}^{c}, \cdots, H_{N}^{c}\right\}$ such that $H_{i_{k}}^{c} \| H_{i_{l}}^{c}$ for all $1 \leqslant k<l \leqslant m$. We will generalize the separation algorithm of one branch to that of multiple branches. Roughly speaking, we give an algorithm to eliminate all $S_{j}^{c} \in G \mid G^{*}$ satisfying $H_{j}^{c} \leqslant H_{i_{k}}^{c}$ for some $H_{i_{k}}^{c} \in I$.

Definition 29. $\bar{I}:=\left\{H_{j}^{c}: H_{j}^{c} \leqslant H_{i}^{c}\right.$ for some $\left.H_{i}^{c} \in I\right\}$.

Theorem 2. ([A.4,A.5.4]) Let $\mathbf{I}=\left\{\left[S_{i_{1}}^{c}\right\rceil_{I}, \cdots,\left\lceil S_{i_{m}}^{c}\right\rceil_{I}\right\}$. Then there exist one closed hypersequent $G_{I}^{\text {in }} \subseteq_{c} G \mid G^{*}$ and its derivation $\tau_{\mathbf{I}}^{\text {in }}$ from $\left\lceil S_{i_{1}}^{c}\right\rceil_{I}, \ldots,\left\lceil S_{i_{m}}^{c}\right\rceil_{I}$ in $\mathbf{G L}_{\Omega}$ such that

(i) $\tau_{\mathbf{I}}^{\text {is }}$ is constructed by applying elimination rules, say,

$$
\frac{G_{b_{1}}\left|S_{j_{1}}^{c} G_{b_{2}}\right| S_{j_{2}}^{c} \cdots G_{b_{w}} \mid S_{j_{w}}^{c}}{=} \quad\left\{\tau_{\mathbf{I}_{\mathbf{j}}}^{*}\right\rangle
$$


and the fully constraint contraction rules, say $\frac{G_{2}}{\overline{G_{1}}}\left\langle E C_{\Omega}^{*}\right\rangle$, where $1 \leqslant w \leqslant m, H_{j_{k}}^{c} \leftrightarrow H_{j_{l}}^{c}$ for all $1 \leqslant k<l \leqslant w$, $I_{\mathbf{j}}=\left\{H_{j_{1}}^{c}, \cdots, H_{j_{w}}^{c}\right\} \subseteq \bar{I}, \mathcal{I}_{\mathbf{j}}=\left\{S_{j_{1}}^{c}, \cdots, S_{j_{w}}^{c}\right\}, \mathbf{I}_{\mathbf{j}}=\left\{G_{b_{1}}\left|S_{j_{1}}^{c}, \cdots, G_{b_{w}}\right| S_{j_{w}}^{c}\right\}$ and $G_{b_{k}} \mid S_{j_{k}}^{c}$ is closed for all $1 \leqslant k \leqslant w$. Then $H_{i}^{c} \nless H_{j}^{c}$ for all $S_{j}^{c} \in G_{\mathcal{I}_{j}}^{*}$ and $H_{i}^{c} \in I$.

(ii) For all $H \in \bar{\tau}_{\mathbf{I}}^{\bar{\hbar}}$,

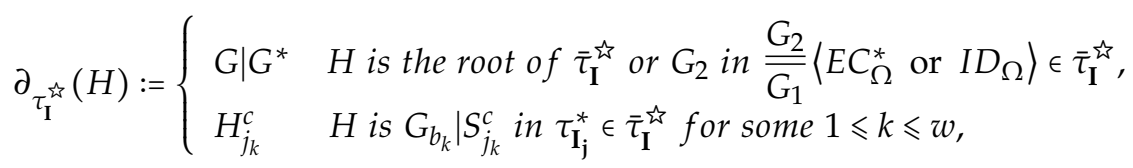

where, $\bar{\tau}_{\mathbf{I}}^{\text {光 }}$ is the skeleton of $\tau_{\mathbf{I}}^{\text {出 }}$ which is defined as Definition 27. Then

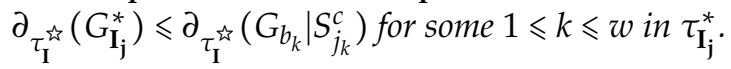

(iii) Let $H \in \bar{\tau}_{\mathbf{I}}^{\text {is }}, G \mid G^{*}<\partial_{\tau_{I}^{\text {in }}}(H) \leqslant H_{I}^{V}$, then $G_{H_{I}^{V}: H}^{\text {is }(J)} \in \tau_{\mathbf{I}}^{\text {is }}$ and it is constructed by applying the separation algorithm along $H_{I}^{V}$ to $H$ and, is an upper hypersequent of either $\left\langle E C_{\Omega}^{*}\right\rangle$ if it is applicable, or $\left\langle I D_{\Omega}\right\rangle$ otherwise.

(iv) $S_{j}^{c} \in G_{\mathbf{I}}^{\xi}$ implies $H_{j}^{c} \| H_{i}^{c}$ for all $H_{i}^{c} \in I$ and, $S_{j}^{c} \in G_{\mathcal{I}_{\mathbf{j}}}^{*}$ for some $\tau_{\mathbf{I}_{\mathbf{j}}}^{*} \in \tau_{\mathbf{I}}^{\text {is }}$ or $S_{j}^{c} \in\left\lceil S_{i_{k}}^{c}\right\rceil_{I}$ for some $H_{i_{k}}^{c} \in I$ satisfying $H_{j}^{c} \nless H_{i_{k}}^{c}$.

Note that in Claim (i), bold $\mathbf{j}$ in $I_{\mathbf{j}}, \mathcal{I}_{\mathbf{j}}$ or $\mathbf{I}_{\mathbf{j}}$ indicates the $w$-tuple $\left(j_{1}, \cdots, j_{w}\right)$ in $S_{j_{1}}^{c}, \cdots, S_{j_{w}}^{c}$. Claim (iv) shows the final aim of Theorem 2, i.e., there exists no $S_{j}^{c} \in G_{\mathbf{I}}^{\tau_{3}}$ such that $H_{j}^{c} \leqslant H_{i}^{c}$ for some $H_{i}^{c} \in I$. It is almost impossible to construct $\tau_{I}^{\text {is }}$ in a non-recursive way. Thus we use Claims (i)-(iii) in Theorem 2 to characterize the structure of $\tau_{\mathbf{I}}^{\tau i}$ in order to construct it recursively.

Proof. $\tau_{I}^{\text {㧒 }}$ is constructed by induction on $|I|$. For the base case, let $|I|=1$. Then $\tau_{I}^{\text {is }}$ is constructed by Construction 5 and 7. Here, Claim (i) holds by Lemma 20(ii), Lemma 18(i) and Lemma 13(vi), Claim (ii) by Lemma 18(i), (iii) is clear and (iv) by Lemma 18(iv).

For the induction case, let $|I| \geqslant 2$. Let $\frac{G^{\prime}\left|S^{\prime} G^{\prime \prime}\right| S^{\prime \prime}}{G^{\prime}\left|G^{\prime \prime}\right| H^{\prime}}(I I) \in \tau^{*}$, where $G^{\prime}\left|G^{\prime \prime}\right| H^{\prime}=H_{I}^{V}$. Then $\left\{H_{i_{1}}^{c}, \cdots, H_{i_{m}}^{c}\right\}$ is divided into two subsets $I_{l}=\left\{H_{l_{1}}^{c}, \cdots, H_{l_{m(l)}}^{c}\right\}, I_{r}=\left\{H_{r_{1}}^{c}, \cdots, H_{r_{m(r)}}^{c}\right\}$, which occur in the left subtree $\tau^{*}\left(G^{\prime} \mid S^{\prime}\right)$ and right subtree $\tau^{*}\left(G^{\prime \prime} \mid S^{\prime \prime}\right)$ of $\tau^{*}\left(H_{I}^{V}\right)$, respectively. Then $m(l)+m(r)=m$. Let $\mathbf{I}_{l}=\left\{\left\lceil S_{l_{1}}^{c}\right\rceil_{I}, \cdots,\left\lceil S_{l_{m(l)}^{c}}^{c}\right\rceil_{I}\right\}, \mathbf{I}_{r}=\left\{\left\lceil S_{r_{1}}^{c}\right\rceil_{I}, \cdots,\left\lceil S_{r_{m(r)}}^{c}\right\rceil_{I}\right\}$. Suppose that derivations $\tau_{\mathbf{I}_{l}}^{\boldsymbol{\xi}}$ of $G_{\mathbf{I}_{l}}^{\xi \xi}$ and $\tau_{\mathbf{I}_{r}}^{\boldsymbol{\xi}}$ of $G_{\mathbf{I}_{r}}^{i j}$ are constructed such that Claims from (i) to (iv) hold. There are three cases to be considered in the following.

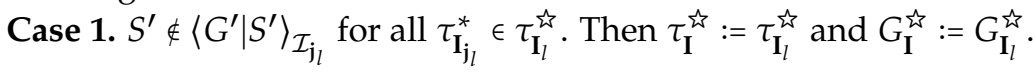

- $\quad$ For Claim (i), let $\tau_{\mathbf{I}_{\mathbf{j}_{l}}}^{*} \in \tau_{\mathbf{I}_{l}}^{\text {光}}$ and $S_{j}^{c} \in G_{\mathcal{I}_{\mathbf{j}_{l}}}^{*}$. By the induction hypothesis, $H_{i}^{c} \nless H_{j}^{c}$ for all $H_{i}^{c} \in I_{l}$. Since $S^{\prime} \notin\left\langle G^{\prime} \mid S^{\prime}\right\rangle_{\mathcal{I}_{\mathbf{j}_{l}}}$ then $G^{\prime \prime} \mid H^{\prime} \cap\left\langle G^{\prime}\left|G^{\prime \prime}\right| H^{\prime}\right\rangle_{\mathcal{I}_{\mathbf{j}_{l}}}=\varnothing$. Thus $G_{H_{I}^{V}: G^{\prime \prime} \mid H^{\prime}}^{*} \cap G_{\mathcal{I}_{\mathbf{j}_{l}}}^{*}=\varnothing$ by Lemmas 11 and 12 . Then $S_{j}^{c} \notin G_{H_{I}^{V}: G^{\prime \prime} \mid H^{\prime}}^{*}$. Thus $G^{\prime \prime} \mid S^{\prime \prime} \nless H_{j}^{c}$ by Proposition 2(i). Hence, for all $H_{i}^{c} \in I_{r}, H_{i}^{c} \nless H_{j}^{c}$ by $G^{\prime \prime} \mid S^{\prime \prime} \leqslant H_{i}^{c}$. Then $H_{i}^{c} \nless H_{j}^{c}$ for all $H_{i}^{c} \in I$. Claims (ii) and (iii) follow directly from the induction hypothesis.

- $\quad$ For Claim (iv), let $S_{j}^{c} \in G_{\mathrm{I}}^{\xi}$. It follows from the induction hypothesis that $H_{j}^{c} \| H_{i}^{c}$ for all $H_{i}^{c} \in I_{l}$ and, $S_{j}^{c} \in G_{\mathcal{I}_{\mathbf{j}_{l}}}^{*}$ for some $\tau_{\mathbf{I}_{\mathbf{j}_{l}}}^{*} \in \tau_{\mathbf{I}_{l}}^{\hbar_{3}}$ or $S_{j}^{c} \in\left\lceil S_{l_{k}}^{c}\right\rceil_{I}$ for some $H_{l_{k}}^{c} \in I_{l}, H_{j}^{c} \nless H_{l_{k}}^{c}$. Then $H_{j}^{c} \nless H_{I}^{V}$ by $H_{j}^{c} \| H_{l_{1}}^{c}, H_{I}^{V}<H_{l_{1}}^{c}$.

If $S_{j}^{c} \in\left[S_{l_{k}}^{c}\right\rceil_{I}$ for some $H_{l_{k}}^{c} \in I_{l}, H_{j}^{c} \nless H_{l_{k}}^{c}$ then $H_{j}^{c} \| H_{i}^{c}$ for all $H_{i}^{c} \in I$ by the definition of branches to $I$. Thus we assume that $S_{j}^{c} \in G_{\mathcal{I}_{\mathbf{j}_{l}}}^{*}$ for some $\tau_{\mathbf{I}_{\mathbf{j}_{l}}}^{*} \in \tau_{\mathbf{I}_{l}}^{\tau_{l}}$ in the following. If $G^{\prime} \mid S^{\prime} \leqslant H_{j}^{c}$ then $H_{j}^{c} \| H_{i}^{c}$ for all $H_{i}^{c} \in I_{r}$ thus $H_{j}^{c} \| H_{i}^{c}$ for all $H_{i}^{c} \in I$. Thus let $G^{\prime} \mid S^{\prime} \nless H_{j}^{c}$ in the following. By the proof of Claim (i) above, $G^{\prime \prime} \mid S^{\prime \prime} \nless H_{j}^{c}$. Then $H_{I}^{V} \nless H_{j}^{c}$ by $G^{\prime} \mid S^{\prime} \nless H_{j}^{c}$ and $G^{\prime \prime} \mid S^{\prime \prime} \nless H_{j}^{c}$. Thus $H_{j}^{c} \| H_{I}^{V}$. Hence $H_{j}^{c} \| H_{i}^{c}$ for all $H_{i}^{c} \in I$. 


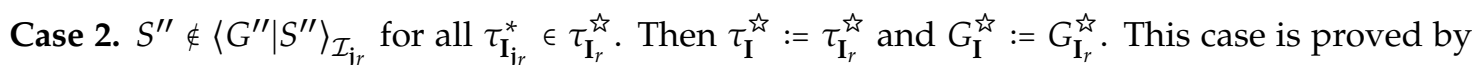
a procedure similar to that of Case 1 and omitted.

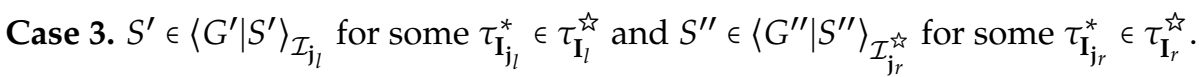

Given

$$
\frac{G_{b_{r 1} 1}\left|S_{j_{r 1}}^{c} G_{b_{r 2}}\right| S_{j_{r 2}}^{c} \cdots G_{b_{r v}} \mid S_{j_{r v}}^{c}}{G_{r} \equiv\left\{G_{b_{r k}}\right\}_{k=1}^{v} \mid G_{\mathcal{I}_{\mathbf{j}}}^{*}}\left|\tau_{\mathbf{I}_{\mathbf{j}}}^{*}\right\rangle \in \tau_{\mathbf{I}_{r}}^{\tilde{\Sigma}}
$$

such that $S^{\prime \prime} \in\left\langle G^{\prime \prime} \mid S^{\prime \prime}\right\rangle_{\mathcal{I}_{\mathrm{j} r}}$ and $H_{j_{r k}}^{c}>H_{I}^{V}$ for all $1 \leqslant k \leqslant v$, where, $1 \leqslant v \leqslant m(r), G_{b_{r k}} S_{j_{r k}}^{c}$ is closed for all $1 \leqslant k \leqslant v, I_{\mathbf{j}_{r}}=\left\{H_{j_{r 1}}^{c}, H_{j_{r 2}}^{c}, \cdots, H_{j_{r v}}^{c}\right\} \subseteq \overline{I_{r}}, \mathcal{I}_{\mathbf{j}_{r}}=\left\{S_{j_{r 1}}^{c}, S_{j_{r_{2}}}^{c}, \cdots, S_{j_{r v}}^{c}\right\}, \mathbf{I}_{\mathbf{j}_{r}}=\left\{G_{b_{r 1} 1}\left|S_{j_{r 1}}^{c}, \cdots, G_{b_{r v}}\right| S_{j_{r v}}^{c}\right\}$. Then $H_{I_{\mathrm{j}_{r}}}^{V} \geqslant G^{\prime \prime} \mid S^{\prime \prime}$ by $I_{\mathbf{j}_{r}} \subseteq \overline{I_{r}}$ and $H_{j_{r k}}^{c}>H_{I}^{V}$ for all $1 \leqslant k \leqslant v$. Thus $H_{j}^{c} \leadsto H_{i}^{c}$ for all $H_{j}^{c} \in I_{\mathbf{j}_{r}}$ and $H_{i}^{c} \in I_{l}$ by $S^{\prime \prime} \in\left\langle G^{\prime \prime} \mid S^{\prime \prime}\right\rangle_{\mathcal{I}_{\mathrm{j}},}$ and Construction 4 .

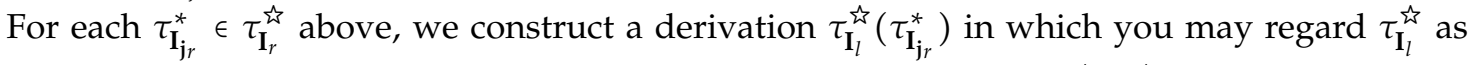

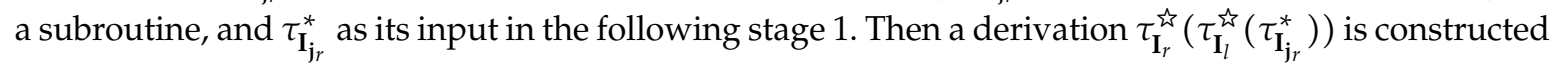

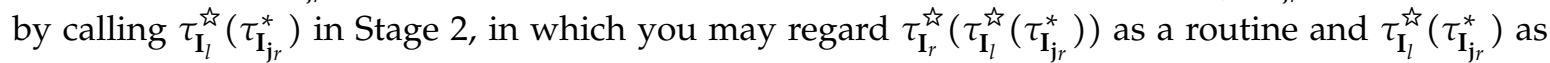
its subroutine.

Before proceeding to deal with Case 3, we present the following property of $\tau_{\mathbf{I}}^{\text {is }}$ which are derived from Claims (i) (iv) and applicable to $\tau_{\mathbf{I}_{l}}^{\hbar_{j}}$ or $\tau_{\mathbf{I}_{r}}^{\tau_{\hat{j}}}$ under the induction hypothesis.

Notation 9. Let

$$
\begin{aligned}
& G_{+}:=\widehat{S^{\prime \prime}}\left|G_{H_{I}^{V}: G^{\prime \prime}}^{\grave{2}(J)}\right| G_{H_{I}^{V}: H^{\prime}}^{\grave{2}(J)} \backslash\left\{\widehat{S^{\prime}} \mid \widehat{S^{\prime \prime}}\right\} \text { and }
\end{aligned}
$$

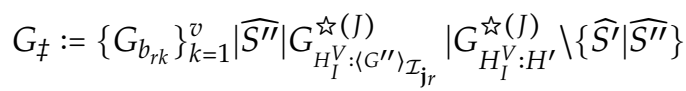

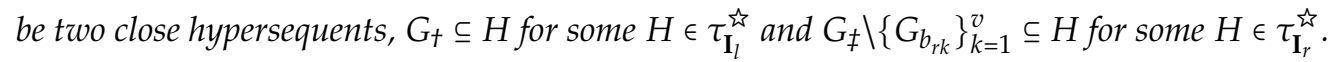

Generally, $\widehat{S^{\prime \prime}} \subseteq G_{+}$is a copy of $\widehat{S^{\prime \prime}} \subseteq G_{\ddagger}$, i.e., eigenvariables in $\widehat{S^{\prime \prime}} \subseteq G_{+}$have different identification numbers with those in $\widehat{S^{\prime \prime}} \subseteq G_{\ddagger}$, so are $H^{\prime}, G^{\prime \prime}, S^{\prime}$.

Lemma 24. $S_{j}^{c} \in G_{+}$implies $H_{j}^{c} \| G^{\prime} \mid S^{\prime}$.

Proof. Let $S_{j}^{c} \in G_{+} \subseteq G_{H_{I}^{V}: G^{\prime \prime} \mid H^{\prime}}^{\mathfrak{i n}(J)}$. Then $H_{j}^{c} \nless H_{I}^{V}$ by Lemma 19(i). Thus $H_{j}^{c}>H_{I}^{V}$ or $H_{j}^{c} \| H_{I}^{V}$. If $H_{j}^{c} \| H_{I}^{V}$ then $H_{j}^{c} \| G^{\prime} \mid S^{\prime}$ by $H_{I}^{V}<G^{\prime} \mid S^{\prime}$ and Proposition 1 (ii). If $H_{j}^{c}>H_{I}^{V}$ then $S_{j}^{c} \in H_{I}^{V}$ by Proposition 2(i). Thus $S_{j}^{c} \in G^{\prime \prime}$ by Lemma 11, Lemma 14(i). Hence $H_{j}^{c} \| G^{\prime} \mid S^{\prime}$ by $H_{j}^{c} \geqslant G^{\prime \prime}\left|S^{\prime \prime}, G^{\prime}\right| S^{\prime} \| G^{\prime \prime} \mid S^{\prime \prime}$.

Lemma 25. (1) $\bar{\tau}_{\mathbf{I}}^{\hbar^{3}}$ is an m-ary tree and, $\tau_{\mathbf{I}}^{\tau_{3}^{2}}$ is a binary tree;

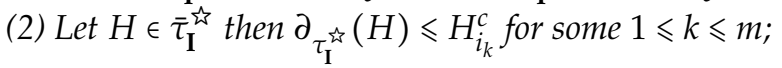

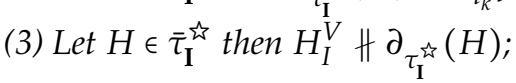

(4) Let $w>1$ in $\tau_{\mathbf{I}_{\mathbf{j}}}^{*} \in \tau_{\mathbf{I}}^{\text {永 }}$ then $H_{I}^{V}<H_{j_{k}}^{c}$ for all $1 \leqslant k \leqslant w$.

(5) Let $\tau_{\mathbf{I}_{\mathbf{j}}}^{*} \in \tau_{\mathbf{I}}^{\tau^{2}}, \partial_{\tau_{\mathbf{I}}^{i \mathbf{j}}}\left(G_{b_{k}} \mid S_{j_{k}}^{c}\right) \leqslant H_{I}^{V}$ for some $1 \leqslant k \leqslant w$. Then $w=1$.

Proof. (1) is immediately from Claim (i). (2) holds by $G \mid G^{*} \leqslant H_{j_{k}}^{c}$ and $H_{j_{k}}^{c} \leqslant H_{i_{k}}^{c}$ for some $H_{i_{k}}^{c} \in I$ by $I_{\mathbf{j}} \subseteq \bar{I}$. (3) holds by Proposition 1(iii), (2) and $H_{I}^{V} \leqslant H_{i_{k}}^{c}$.

For (4), let $w>1$. Then $H_{j_{1}}^{c} \| H_{j_{k}}^{c}$ for each $2 \leqslant k \leqslant w, H_{j_{1}}^{c} \leqslant H_{i_{g}}^{c}$ and $H_{j_{k}}^{c} \leqslant H_{i_{h}}^{c}$ for some $H_{i_{g}}^{c} H_{i_{h}}^{c} \in I$ by (2). Thus $H_{j_{1}}^{c} \| H_{i_{h}}^{c}$ and $H_{j_{k}}^{c} \| H_{i_{g}}^{c}$ by Proposition 1(ii). Hence $H_{j_{1}}^{c} \nless H_{i_{g} i_{h}}^{V}$ by $H_{i_{g} i_{h}}^{V}<H_{i_{h}}^{c}$, and $H_{j_{k}}^{c} \nless H_{i_{g} i_{h}}^{V}$ by $H_{i_{g} i_{h}}^{V}<H_{i_{g}}^{c}$. Thus $H_{I}^{V}<H_{j_{1}}^{c}$ and $H_{I}^{V}<H_{j_{k}}^{c}$ by (3), $H_{I}^{V} \leqslant H_{j_{1} j_{k}}^{V}$. Hence $H_{I}^{V}<H_{j_{k}}^{c}$ for all $1 \leqslant k \leqslant w$. (5) is from (4). 


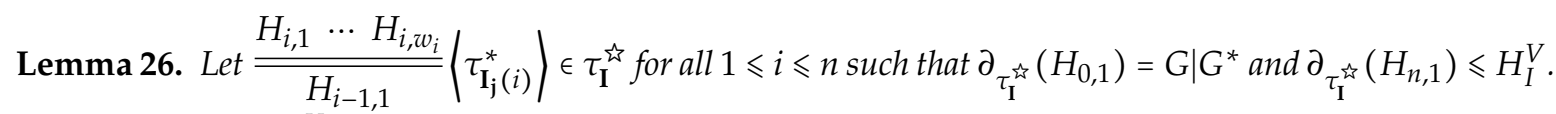
Then $\partial_{\tau_{\mathrm{I}}^{\hat{\jmath}}}\left(H_{i, 1}\right) \leqslant H_{I}^{V}$ and $w_{i}=1$ for all $1 \leqslant i \leqslant n$.

Proof. The proof is by induction on $n$. Let $n=1$ then $w_{1}=1$ by Lemma 25(5) and $\partial_{\tau_{I}^{\hat{\Lambda}}}\left(H_{1,1}\right) \leqslant H_{I}^{V}$. For the induction step, let $\partial_{\tau_{I}^{i,}}\left(H_{i, 1}\right) \leqslant H_{I}^{V}$ for some $1<i \leqslant n$ then $w_{i}=1$ by Lemma 25(5). Since $\frac{H_{i, 1} \cdots H_{i, w_{i}}}{H_{i-1,1}}\left|\tau_{\mathbf{I}_{\mathbf{j}}(i)}^{*}\right\rangle \in \tau_{\mathbf{I}}^{\text {负 }}$ then $\partial_{\tau_{\mathbf{I}}^{\hat{\mathbf{I}}}}\left(H_{i-1,1}\right) \leqslant \partial_{\tau_{\mathbf{I}}^{\hat{\mathbf{I}}}}\left(H_{i, k}\right)$ for some $1 \leqslant k \leqslant w_{i}$ by Claim (ii). Then $\partial_{\tau_{\mathbf{I}}^{\natural}}\left(H_{i-1,1}\right) \leqslant \partial_{\tau_{\mathbf{I}}^{\natural}}\left(H_{i, 1}\right) \leqslant H_{I}^{V}$ by $w_{i}=1$. Thus $w_{i-1}=1$ by Lemma $25(5)$.

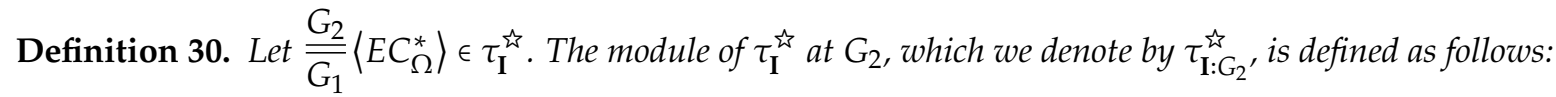

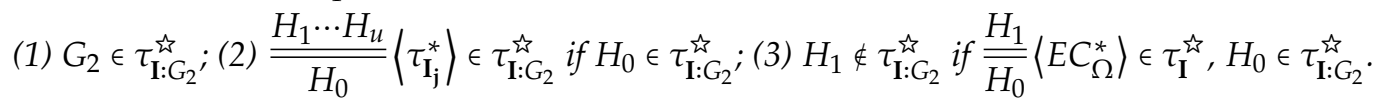

Each node of $\tau_{\mathrm{I}: G_{2}}^{i j}$ is determined bottom-up, starting with $G_{2}$, whose root is $G_{2}$ and leaves may be branches, leaves of $\tau^{*}$ or lower hypersequents of $\left\langle E C_{\Omega}^{*}\right\rangle$-applications. While each node of $\tau_{H: H^{\prime}}^{*}$ is determined top-down, starting with $H^{\prime}$, whose root is a subset of $G \mid G^{*}$ and leaves contain $H^{\prime}$ and some leaves of $\tau^{*}$.

Lemma 27. (1) $\tau_{\mathbf{I}: G_{2}}^{\sum_{3}}$ is a derivation without $\left\langle E C_{\Omega}^{*}\right\rangle$ in $\mathbf{G L}_{\Omega}$.

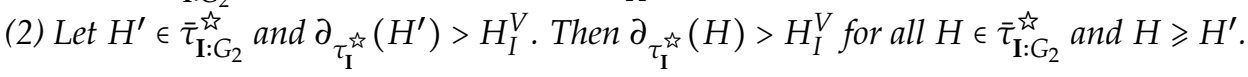

Proof. Part (1) is clear and (2) immediately follows from Lemma 26.

Now, we continue to deal with Case 3 in the following.

Stage 1 Construction of Subroutine $\tau_{\mathbf{I}_{l}}^{\hbar_{2}}\left(\tau_{\mathbf{I}_{\mathbf{j}}}^{*}\right)$. Roughly speaking, $\tau_{\mathbf{I}_{l}}^{\hbar_{3}}\left(\tau_{\mathbf{I}_{\mathbf{j}_{r}}}^{*}\right)$ is constructed by replacing some nodes $\tau_{\mathbf{I}_{\mathbf{j}_{l}}}^{*} \in \tau_{\mathbf{I}_{l}}^{\boldsymbol{I}_{l}}$ with $\tau_{\mathbf{I}_{\mathbf{j}_{l}} \cup \mathbf{I}_{\mathbf{j} r}}^{*}$ in post-order. However, the ordinal postorder-traversal algorithm cannot be used to construct $\tau_{\mathbf{I}_{l}}^{\hbar_{j}}\left(\tau_{\mathbf{I}_{\mathbf{j}_{r}}}^{*}\right)$ because the tree structure of $\tau_{\mathbf{I}_{l}}^{\text {in }}\left(\tau_{\mathbf{I}_{\mathbf{j}_{r}}}^{*}\right)$ is generally

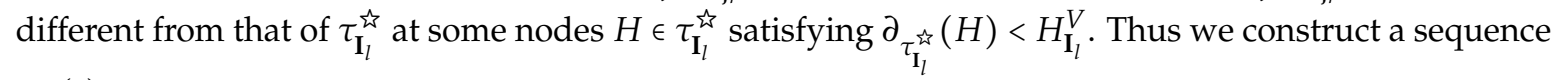
$\tau_{\mathbf{I}_{l}}^{\text {光 }(q)}$ of trees for all $q \geqslant 0$ inductively as follows.

For the base case, we mark all $\left\langle E C_{\Omega}^{*}\right\rangle$-applications in $\tau_{\mathbf{I}_{l}}^{\text {出 }}$ as unprocessed and define such marked derivation to be $\tau_{\mathbf{I}_{l}}^{i(0)}$. For the induction case, let $\tau_{\mathbf{I}_{l}}^{i(q)}$ be constructed. If all applications of $\left\langle E C_{\Omega}^{*}\right\rangle$ in $\tau_{\mathbf{I}_{I}}^{\tilde{\tau}(q)}$ are marked as processed, we firstly delete the root of the tree resulting from the procedure and then, apply $\left\langle E C_{\Omega}^{*}\right\rangle$ to the root of the resulting derivation if it is applicable otherwise add an $\left\langle I D_{\Omega}\right\rangle$-application to it and finally, terminate the procedure. Otherwise we select one of the outermost unprocessed $\left\langle E C_{\Omega}^{*}\right\rangle$-applications in $\tau_{\mathbf{I}_{l}}^{i(q)}$, say, $\frac{G_{q+1}^{\circ}}{\overline{G_{q+1}^{\circ}}}\left\langle E C_{\Omega}^{*}\right\rangle_{q+1^{\prime}}^{\circ}$, and perform the following steps to construct $\tau_{\mathbf{I}_{l}}^{\dot{\sim}(q+1)}$ in which $\frac{G_{q+1}^{\circ \circ}}{\overline{G_{q+1}^{\circ}}}\left\langle E C_{\Omega}^{*}\right\rangle_{q+1}^{\circ}$ be revised as $\frac{G_{q+1}^{*}}{\overline{G_{q+1}^{\circ}}}\left\langle E C_{\Omega}^{*}\right\rangle_{q+1}^{*}$ such that

(a) $\tau_{\mathbf{I}_{l}}^{i j(q+1)}$ is constructed by locally revising $\tau_{\mathbf{I}_{l}: G_{q+1}^{\circ}}^{i(q)}$ and leaving other nodes of $\tau_{\mathbf{I}_{l}}^{i(q)}$ unchanged, particularly including $G_{q+1}^{\circ}$;

(b) $\tau_{\mathbf{I}_{l}}^{\grave{2}(q+1)}\left(G_{q+1}^{\ddot{*}}\right)$ is a derivation in $\mathbf{G L}_{\Omega}$; 
(c) $G_{q+1}^{*}=G_{q+1}^{\circ \circ}$ if $S^{\prime} \notin\left\langle G^{\prime} \mid S^{\prime}\right\rangle_{\mathcal{I}_{\mathbf{j}_{l}}}$ for all $\tau_{\mathbf{I}_{\mathbf{j}_{l}}}^{*} \in \tau_{\mathbf{I}_{l}}^{\boldsymbol{\zeta}_{l}}\left(G_{q+1}^{\circ \circ}\right)$ otherwise $G_{q+1}^{*}=G_{q+1}^{\circ \circ} \backslash G_{+}^{m_{q+1}} \mid G_{\ddagger}^{m_{q+1}}$ for some $m_{q+1} \geqslant 1$.

Remark 3. By two superscripts $\circ$ and $\cdot$ in $\left\langle E C_{\Omega}^{*}\right\rangle_{q+1}^{\circ}$ or $\left\langle E C_{\Omega}^{*}\right\rangle_{q+1}$, we indicate the unprocessed state and processed state, respectively. This procedure determines an ordering for all $\left\langle E C_{\Omega}^{*}\right\rangle$-applications in $\tau_{\mathbf{I}_{l}}^{\hbar_{1}}$ and the subscript $q+1$ indicates that it is the $q+1$-th application of $\left\langle E C_{\Omega}^{*}\right\rangle$ in a post-order transversal of $\tau_{\mathbf{I}_{l}}^{\boldsymbol{\nu}_{\mathbf{1}}} . G_{q+1}^{\circ o}$ and $G_{q+1}^{\circ}\left(G_{q+1}^{*}\right.$ and $\left.G_{q+1}^{\cdot}\right)$ are the premise and conclusion of $\left\langle E C_{\Omega}^{*}\right\rangle_{q+1}^{\circ}\left(\left\langle E C_{\Omega}^{*}\right\rangle_{q+1}^{\cdot}\right)$, respectively.

Step 1 (Delete). Take the module $\tau_{\mathbf{I}_{l}: G_{q+1}^{\circ}}^{i(q)}$ out of $\tau_{\mathbf{I}_{l}}^{i j(q)}$. Since $\left\langle E C_{\Omega}^{*}\right\rangle_{q+1}^{\circ}$ is the unique unprocessed

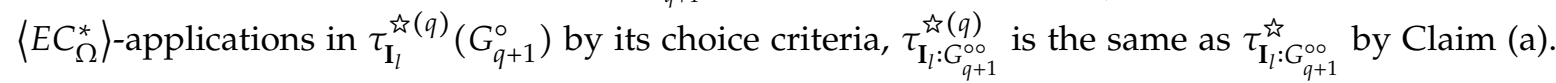

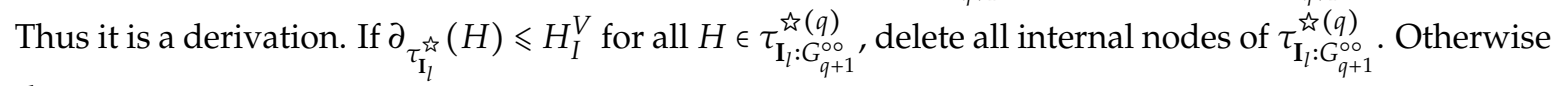
there exists

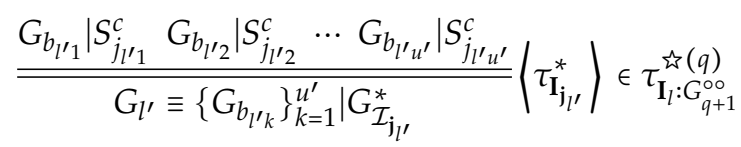

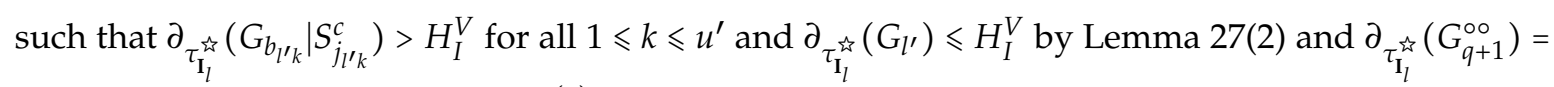
$G \mid G^{*} \leqslant H_{I}^{V}$, then delete all $H \in \tau_{\mathbf{I}_{l}: G_{q+1}^{\circ}}^{i(q)}, G_{q+1}^{\circ \circ} \leqslant H<G_{l^{\prime}}$. We denote the structure resulting from the

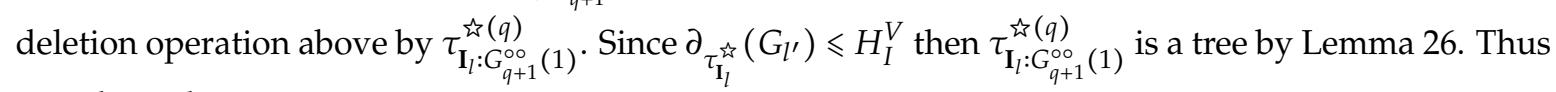
it is also a derivation.

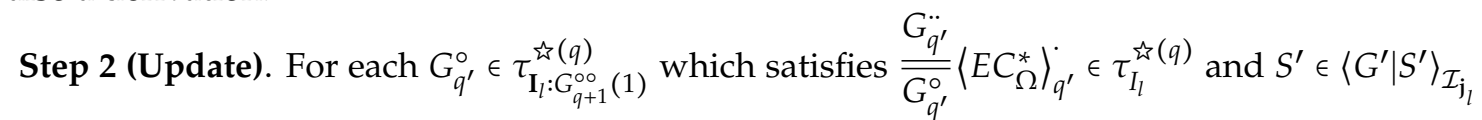

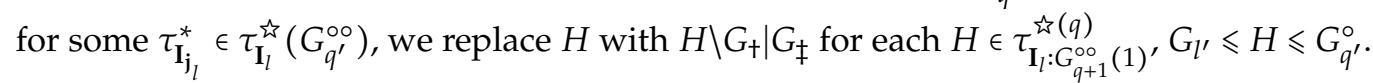

Since $\frac{G_{q^{\prime}}^{\prime \prime}}{\overline{G_{q^{\prime}}^{\circ}}}\left\langle E C_{\Omega}^{*}\right\rangle_{q^{\prime}}^{*} \in \tau_{I_{l}}^{i(q)}\left(G_{q+1}^{\circ \circ}\right)$ and $\left\langle E C_{\Omega}^{*}\right\rangle_{q+1}^{\circ}$ is the outermost unprocessed $\left\langle E C_{\Omega}^{*}\right\rangle$-application in $\tau_{\mathbf{I}_{l}}^{\hbar(q)}$ then $q^{\prime} \leqslant q$ and $\left\langle E C_{\Omega}^{*}\right\rangle_{q^{\prime}}$, has been processed. Thus Claims (b) and (c) hold for $\tau_{\mathbf{I}_{l}}^{i j(q)}\left(G_{q^{\prime}}^{\cdot}\right)$ by the induction hypothesis. Then $\frac{G_{q^{\prime}}^{*}}{\overline{G_{q^{\prime}}^{*}}}$ is a valid $\left\langle E C_{\Omega}^{*}\right\rangle$-application since $\frac{G_{q^{\prime}}^{\circ \circ}}{\overline{G_{q^{\prime}}^{\circ}}}, \frac{G_{+}^{m_{q^{\prime}}}}{\overline{G_{+}}}$and $\frac{G_{\ddagger}^{m_{q^{\prime}}}}{\overline{G_{\ddagger}}}$ are valid, where $G_{q^{\prime}}^{\ddot{*}}=G_{q^{\prime}}^{\circ \circ} \backslash G_{\dagger}^{m_{q^{\prime}}}\left|G_{\ddagger}^{m_{q^{\prime}}}, G_{q^{\prime}}^{\circ}=G_{q^{\prime}}^{\circ} \backslash G_{+}\right| G_{\ddagger}$.

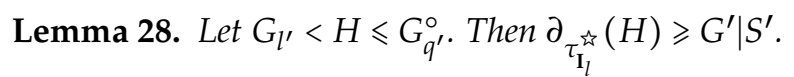

Proof. Since $G_{l^{\prime}}<H$ then $G_{b_{l^{\prime} k}} \mid S_{j_{l^{\prime} k}}^{c} \leqslant H$ for some $1 \leqslant k \leqslant u^{\prime}$. If $\partial_{\tau_{\mathbf{I}_{l}^{\natural}}^{\hat{\gamma}}}(H) \geqslant H_{I_{l}}^{V}$ then $\partial_{\tau_{\mathbf{I}_{l}}^{\hat{\gamma}}}(H) \geqslant G^{\prime} \mid S^{\prime}$.

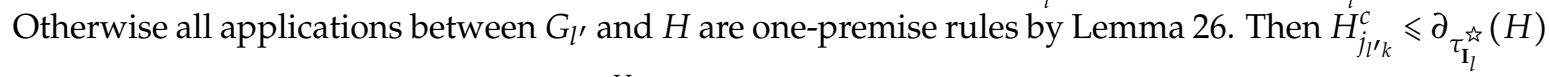

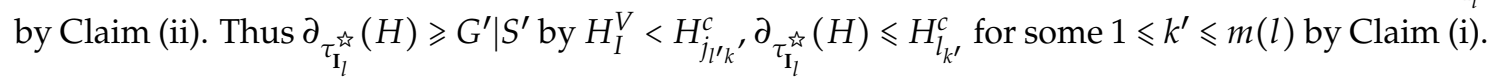

Since $\partial_{\tau_{I_{l}}^{\lambda_{1}}}(H) \geqslant G^{\prime} \mid S^{\prime}$ by Lemma 28 and $H_{j}^{c} \| G^{\prime} \mid S^{\prime}$ for each $S_{j}^{c} \in G_{+}$by Lemma 24 , then $G_{+} \subseteq H$ as side-hypersequent of $H$. Thus this step updates the revision of $G_{q^{\prime}}$, downward to $G_{l^{\prime}}$. 
Let $m^{\prime}$ be the number of $G_{q^{\prime}}^{\circ}$ satisfying the above conditions, $\tau_{\mathbf{I}_{l}: G_{q^{\prime}}^{\circ}(1)}^{i(q)}, G_{l^{\prime}}$ and $G_{b_{l^{\prime} k} k} \mid S_{j^{\prime} k}^{c}$ for all

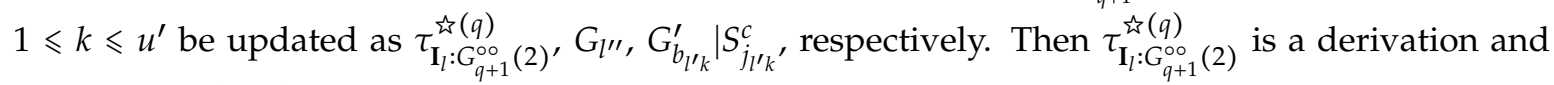
$G_{l^{\prime \prime}}=G_{l^{\prime}} \backslash G_{\dagger}^{m^{\prime}} \mid G_{\ddagger}^{m^{\prime}}$.

Step 3 (Replace). All $\tau_{\mathbf{I}_{\mathbf{j}_{l}}}^{*} \in \tau_{\mathbf{I}_{l}: G_{q+1}^{\circ}}^{\stackrel{\hbar}{2}(q)}$ are processed in post-order. If $H_{i}^{c} \leadsto H_{j}^{c}$ for all $H_{i}^{c} \in I_{\mathbf{j}_{l}}$ and $H_{j}^{c} \in I_{\mathbf{j}_{r}}$ it proceeds by the following procedure otherwise it remains unchanged. Let $\tau_{\mathbf{I}_{\mathbf{j}_{l}}}^{*}$ be in the form

$$
\frac{G_{b_{l 1}}\left|S_{j_{l 1}}^{c} G_{b_{l 2}}\right| S_{j_{l 2}}^{c} \cdots G_{b_{l u}} \mid S_{j_{l u}}^{c}}{G_{l} \equiv\left\{G_{b_{l k}}\right\}_{k=1}^{u} \mid G_{\mathcal{I}_{j_{l}}}^{*}} .
$$

Then $H_{j_{l k}}^{c} \geqslant G^{\prime} \mid S^{\prime}$ for all $1 \leqslant k \leqslant u$ by Lemma $28, G_{b_{l k}} \mid S_{j_{l k}}^{c}>G_{l^{\prime \prime}}$.

Firstly, replace $\tau_{\mathbf{I}_{\mathbf{j}_{l}}}^{*}$ with $\tau_{\mathbf{I}_{\mathbf{j}_{l}} \cup \mathbf{I}_{\mathbf{j}_{r}}}^{*}$. We may rewrite the roots of $\tau_{\mathbf{I}_{\mathbf{j}_{l}}}^{*}$ and $\tau_{\mathbf{I}_{\mathbf{j}_{l}}}^{*} \cup \mathbf{I}_{\mathbf{j}_{r}}$ as

$$
\begin{aligned}
& G_{l}=\left\{G_{b_{l k}}\right\}_{k=1}^{u}\left|G_{H_{I}^{V}:\left\langle G^{\prime}\right\rangle_{\mathcal{I}_{j_{l}}}}\right| G_{H_{I}^{V}: G^{\prime \prime} \mid H^{\prime}}^{*} \text { and } \\
& G_{l, r} \equiv\left\{G_{b_{l k}}\right\}_{k=1}^{u}\left|G_{H_{I}^{V}:\left\langle G^{\prime}\right\rangle_{\mathcal{I}_{\mathbf{j}_{l}}}}\right|\left\{G_{b_{r k}}\right\}_{k=1}^{v}\left|G_{H_{I}^{V}}^{*}:\left\langle G^{\prime \prime}\right\rangle_{\mathcal{I}_{\mathbf{j}_{r}}}\right| H^{\prime \prime}
\end{aligned}
$$

respectively.

Let $G_{l^{\prime \prime}}<H \leqslant G_{l}$. By Lemma 28, $\partial_{\tau_{I_{l}}}(H) \geqslant G^{\prime} \mid S^{\prime}$. By Lemma $14, H_{j}^{c} \leqslant H_{I}^{V}<G^{\prime} \mid S^{\prime}$ or $H_{j}^{c} \| G^{\prime} \mid S^{\prime}$ for all $S_{j}^{c} \in G_{H_{I}^{V}: G^{\prime \prime} \mid H^{\prime}}^{*}$. Thus $G_{H_{I}^{V}: G^{\prime \prime} \mid H^{\prime}}^{*} \subseteq H$. Secondly, we replace $H$ with $H \backslash G_{H_{I}^{V}: G^{\prime \prime} \mid H^{\prime}}^{*}\left|\left\{G_{b_{r k}}\right\}_{k=1}^{v}\right| G_{H_{I}^{V}:\left\langle G^{\prime \prime}\right\rangle_{\mathcal{I}_{\mathbf{j}}} \mid H^{\prime}}$ for all $G_{l^{\prime}} \leqslant H \leqslant G_{l}$. Let $m^{\prime \prime}$ be the number of $\tau_{\mathbf{I}_{j_{l}}}^{*} \in \tau_{\mathbf{I}_{l}: G_{q+1}^{\circ o}(2)}^{\Omega(q)}$ satisfying the replacement conditions

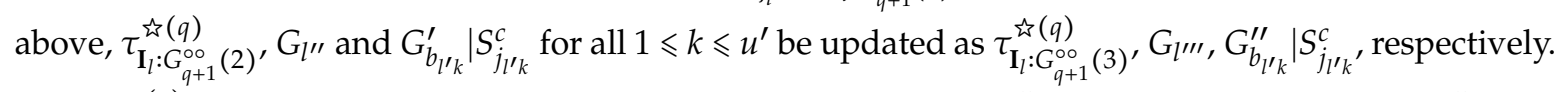

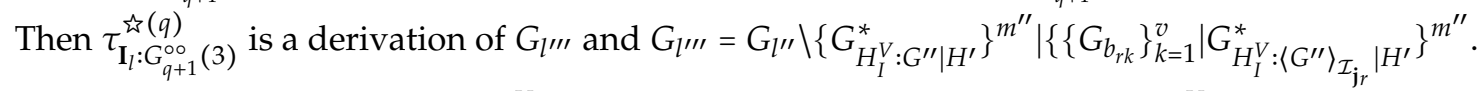

Step 4 (Separation along $H_{I}^{V}$ ). Apply the separation algorithm along $H_{I}^{V}$ to $G_{l^{\prime \prime \prime}}$ and denote the

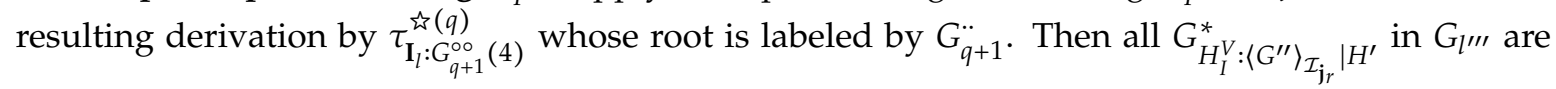

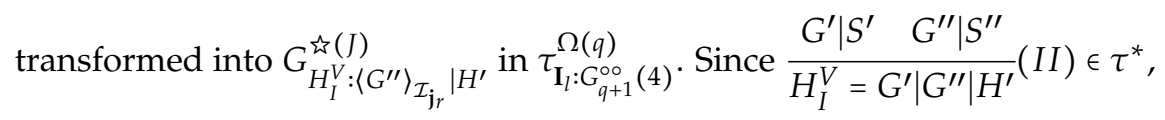

$$
\frac{\left\{G_{b_{l k}}\right\}_{k=1}^{u}\left|\left\langle G^{\prime} \mid S^{\prime}\right\rangle_{\mathcal{I}_{j_{l}}} \quad\left\{G_{b_{r k}}\right\}_{k=1}^{v}\right|\left\langle G^{\prime \prime} \mid S^{\prime \prime}\right\rangle_{\mathcal{I}_{\mathbf{j}_{r}}}}{\left\{G_{b_{l k}}\right\}_{k=1}^{u}\left|\left\{G_{b_{r k}}\right\}_{k=1}^{v}\right|\left\langle G^{\prime}\right\rangle_{\mathcal{I}_{\mathbf{j}_{l}}}\left|\left\langle G^{\prime \prime}\right\rangle_{\mathcal{I}_{\mathbf{j}_{r}}}\right| H^{\prime}}(I I) \in \tau_{\mathbf{I}_{\mathbf{j}_{l}} \cup_{\mathbf{j}_{r}}}^{*} \in \tau_{\mathbf{I}_{l}: G_{q+1}^{\circ}}^{\mathcal{\imath}(q)}(3)^{\prime}
$$

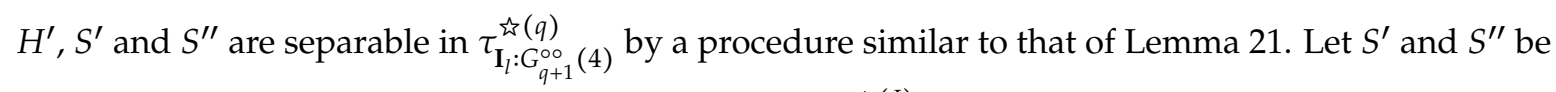
separated into $\widehat{S^{\prime}}$ and $\widehat{S^{\prime \prime}}$, respectively. By Claim (iii), $G_{H_{I}^{V}: G_{l^{\prime}}}^{i(J)}=G_{q+1}^{\circ}$. 


$$
\begin{aligned}
& G_{H_{I}^{V}: G_{l^{\prime \prime}}}^{\mathfrak{\imath}(J)}=G_{q+1}^{\circ \circ} \backslash G_{\ddagger}^{m^{\prime}} \mid G_{\ddagger}^{m^{\prime}} \text { by Lemma 19(iv), } \\
& G_{q+1}^{*}=G_{H_{I}^{V}: G_{l^{\prime \prime \prime}}}^{\mathfrak{k}(J)}
\end{aligned}
$$

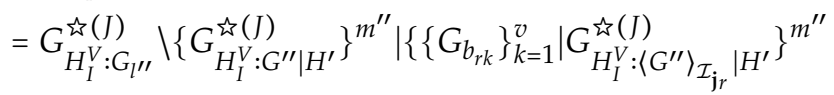

$$
\begin{aligned}
& =G_{H_{I}^{V}: G_{l^{\prime \prime}}}^{\grave{2}(J)} \backslash\left\{\widehat{S^{\prime}}\left|\widehat{S^{\prime \prime}}\right| G_{H_{I}^{V}: G^{\prime \prime}}^{\mathfrak{2}(J)} \mid G_{H_{I}^{V}: H^{\prime}}^{\grave{2}(J)} \backslash\left\{\widehat{S^{\prime}} \mid \widehat{S^{\prime \prime}}\right\}\right\}^{m^{\prime \prime}} \mid \\
& \left\{\left\{G_{b_{r k}}\right\}_{k=1}^{v}\left|\widehat{S^{\prime}}\right| \widehat{S^{\prime \prime}}\left|G_{H_{I}^{V}:\left\langle G^{\prime \prime}\right\rangle_{\mathcal{I}_{\mathbf{j}}}}^{\mathfrak{\imath}(J)}\right| G_{H_{I}^{V}: H^{\prime}}^{\mathfrak{h}(J)} \backslash\left\{\widehat{S^{\prime}} \mid \widehat{S^{\prime \prime}}\right\}\right\}^{m^{\prime \prime}} \\
& =G_{H_{I}^{V}: G_{l^{\prime \prime}}}^{\mathfrak{i n}(J)} \backslash G_{\dagger}^{m^{\prime \prime}} \mid G_{\ddagger}^{m^{\prime \prime}} \\
& =\left\{G_{q+1}^{\circ \circ} \backslash G_{+}^{m^{\prime}} \mid G_{\ddagger}^{m^{\prime}}\right\} \backslash G_{+}^{m^{\prime \prime}} \mid G_{\ddagger}^{m^{\prime \prime}} \\
& =G_{q+1}^{\circ \circ} \backslash G_{+}^{m^{\prime}+m^{\prime \prime}} \mid G_{\ddagger}^{m^{\prime}+m^{\prime \prime}} \\
& =G_{q+1}^{\circ \circ} \backslash G_{+}^{m_{q+1}} \mid G_{\ddagger}^{m_{q+1}}
\end{aligned}
$$

where $m_{q+1}:=m^{\prime}+m^{\prime \prime}$.

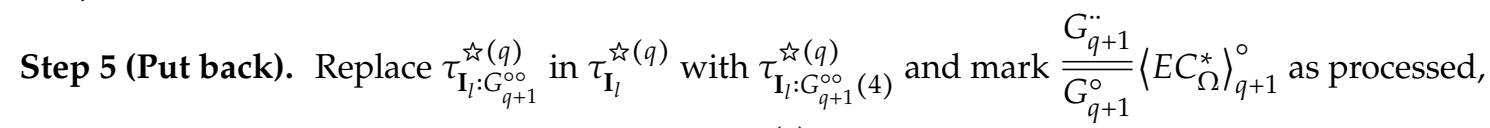
i.e., revise $\left\langle E C_{\Omega}^{*}\right\rangle_{q+1}^{\circ}$ as $\left\langle E C_{\Omega}^{*}\right\rangle_{q+1}$. Among leaves of $\tau_{\mathbf{I}_{l}: G_{q+1}^{\circ}}^{i(q)}$, all $G_{q^{\prime}}^{\circ}$ are updated as $G_{q^{\prime}}^{\cdot}$ and others keep

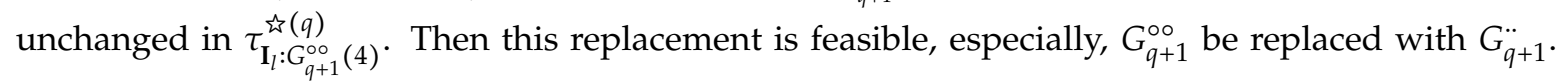
Define the tree resulting from Step 5 to be $\tau_{\mathbf{I}_{l}}^{\mathfrak{h}_{l}(q+1)}$. Then Claims (a), (b) and (c) hold for $q+1$ by the above construction.

Finally, we construct a derivation of $G_{\mathbf{I}_{l}}^{i \vec{i}} \backslash G_{+} \mid G_{\ddagger}$ from $\left\lceil S_{l_{1}}^{c}\right]_{I}, \cdots,\left\lceil S_{l_{m(l)}}^{c}\right]_{I}, G_{b_{r 1}}\left|S_{j_{r 1}}^{c}, \cdots, G_{b_{r v}}\right| S_{j_{r v}}^{c}$ in $\mathrm{GL}_{\Omega}$, which we denote by $\tau_{\mathbf{I}_{l}}^{\hbar_{h}}\left(\tau_{\mathbf{I}_{\mathbf{j}}}^{*}\right)$.

Remark 4. All elimination rules used in constructing $\tau_{\mathbf{I}_{l}}^{\text {出 }}$ are extracted from $\tau^{*}$. Since $\tau_{\mathbf{I}_{j} r}^{*}$ is a derivation in $\mathbf{G L}_{\Omega}$ without $\left(E C_{\Omega}\right)$, we may extract elimination rules from $\tau_{\mathbf{I}_{\mathbf{j}_{r}}}^{*}$ which we may use to construct $\tau_{\mathbf{I}_{l}}^{\lambda_{2}}\left(\tau_{\mathbf{I}_{\mathbf{j}_{r}}}^{*}\right)$ by a procedure similar to that of constructing $\tau_{\mathbf{I}_{l}}^{\text {出 }}$ with minor revision at every node $H$ that $\partial_{\tau_{\mathbf{I}_{l}}^{\text {虫 }}}(H) \leqslant H_{I}^{V}$. Note that updates and replacements in Steps 2 and 3 are essentially inductive operations but we neglect it for simplicity.

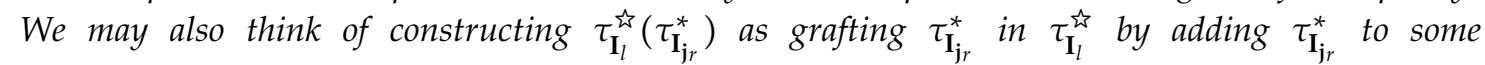

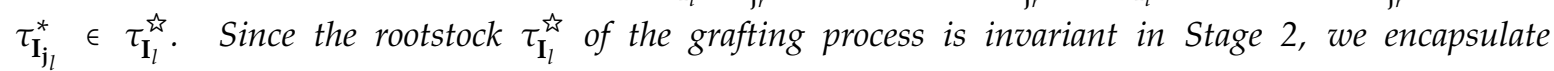
$\tau_{\mathbf{I}_{l}}^{\iota_{i}}\left(\tau_{\mathbf{I}_{j}}^{*}\right)$ as an rule in $\mathbf{G L}_{\Omega}$ whose premises are $G_{b_{r 1}}\left|S_{j_{r 1}}^{c}, G_{b_{r 2}}\right| S_{j_{r 2}}^{c}, \cdots, G_{b_{r v}} \mid S_{j_{r v}}^{c}$ and conclusion is

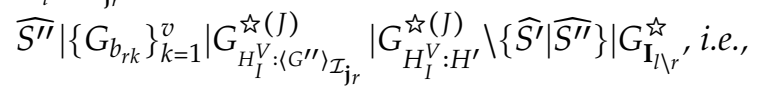

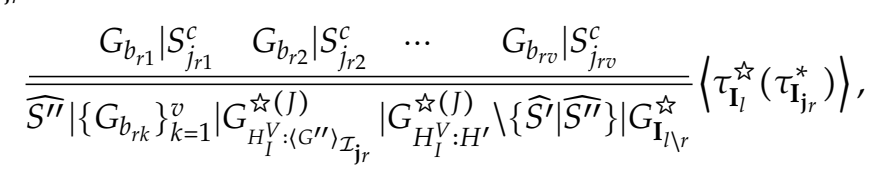

where, $G_{\mathbf{I}_{l \backslash r}}^{i j}=G_{\mathbf{I}_{l}}^{i j} \backslash G_{+}$is closed.

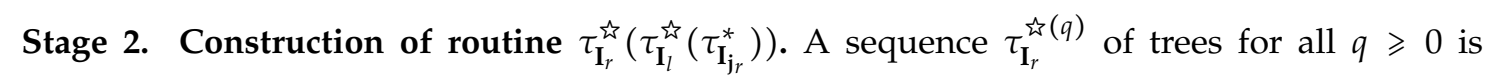

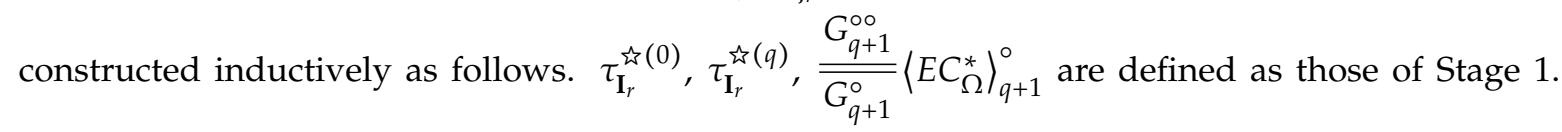


Then we perform the following steps to construct $\tau_{\mathbf{I}_{r}}^{i(q+1)}$ in which $\frac{G_{q+1}^{\circ \circ}}{\overline{G_{q+1}^{\circ}}}\left\langle E C_{\Omega}^{*}\right\rangle_{q+1}^{\circ}$ be revised as $\frac{G_{q+1}^{*}}{\overline{G_{q+1}^{\circ}}}\left\langle E C_{\Omega}^{*}\right\rangle_{q+1}^{.}$such that Claims (a) and (b) are same as those of Stage 1 and (c) $G_{q+1}^{*}=G_{q+1}^{\circ \circ}$ if

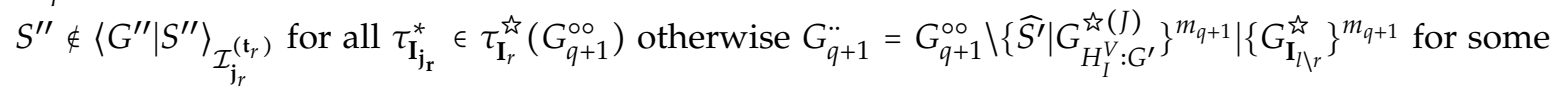
$m_{q+1} \geqslant 1$.

Step 1 (Delete). $\tau_{\mathbf{I}_{r}: G_{q+1}^{\circ}}^{i(q)}$ and $\tau_{\mathbf{I}_{r}: G_{q+1}^{\circ}(q)}^{i(1)}$ are defined as before.

$$
\frac{G_{b_{r^{\prime} 1}}\left|S_{j_{r^{\prime} 1}}^{c} G_{b_{r^{\prime}} 2}\right| S_{j_{r^{\prime} 2}}^{c} \cdots G_{b_{r^{\prime} v^{\prime}}} \mid S_{j_{r^{\prime} v^{\prime}}}^{c}}{G_{r^{\prime}} \equiv\left\{G_{b_{r^{\prime} k}}\right\}_{k=1}^{v^{\prime}} \mid G_{\mathcal{I}_{j_{r^{\prime}}}}^{*}}\left|\tau_{\mathbf{I}_{\mathbf{j}^{\prime}}}^{*}\right| \in \tau_{\mathbf{I}_{r^{\prime}}: G_{q+1}^{\circ}}^{i(q)}
$$

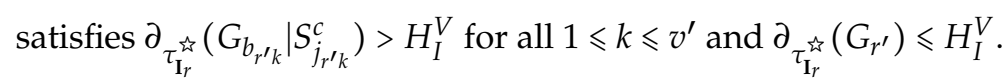

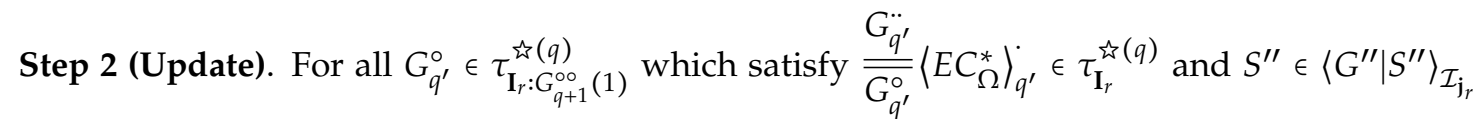

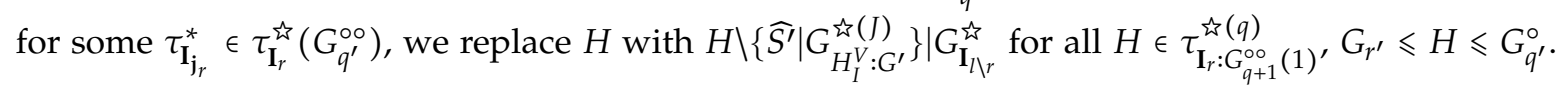
Then Claims (a) and (b) are proved by a procedure as before. Let $m^{\prime}$ be the number of $G_{q^{\prime}}$, satisfying the above conditions. $\tau_{\mathbf{I}_{r}: G_{q+1}^{\circ}(q)}^{i \xi}(1), G_{r^{\prime}}$ and $G_{b_{r^{\prime} k}} \mid S_{j_{r^{\prime} k}}^{c}$ for all $1 \leqslant k \leqslant v^{\prime}$ be updated as $\tau_{\mathbf{I}_{r}: G_{q+1}^{\circ}(2)}^{i(q)}, G_{r^{\prime \prime}}, G_{b_{r^{\prime} k}}^{\prime} \mid S_{j_{r^{\prime}} k^{\prime}}^{c}$, respectively. Then $\tau_{\mathbf{I}_{r^{\prime}}: G_{q+1}^{\circ}(2)}^{i+(q)}$ is a derivation and $G_{r^{\prime \prime}}=G_{r^{\prime}} \backslash\left\{\widehat{S^{\prime}} \mid G_{H_{I}^{V}: G^{\prime}}^{i n(J)}\right\}^{m^{\prime}} \mid\left\{G_{\mathbf{I}_{\lambda r_{r}}}^{i s}\right\}^{m^{\prime}}$.

Step 3 (Replace). All $\tau_{\mathbf{I}_{\mathbf{j}_{r}}^{*}}^{*} \tau_{\mathbf{I}_{r}: G_{q+1}^{\circ o}(2)}^{\Omega(q)}$ are processed in post-order. If $H_{i}^{c} \leadsto H_{j}^{c}$ for all $H_{i}^{c} \in I_{\mathbf{j}_{r}}$ and $H_{j}^{c} \in I_{l}$ it proceeds by the following procedure otherwise it remains unchanged. Let $\tau_{\mathbf{I}_{\mathbf{j} r}}^{*}$ be in the form

$$
\frac{G_{b_{r 1} \mid}\left|S_{j_{r 1}}^{c} G_{b_{r 2}}\right| S_{j_{r 2}}^{c} \cdots G_{b_{r v}} \mid S_{j_{r v}}^{c}}{G_{r} \equiv\left\{G_{b_{r k}}\right\}_{k=1}^{v} \mid G_{\mathcal{I}_{j r}}^{*}} .
$$

Then there exists the unique $1 \leqslant k^{\prime} \leqslant v^{\prime}$ such that $G_{r^{\prime \prime}}<G_{b_{r^{\prime} k^{\prime}}} S_{j_{r^{\prime} k^{\prime}}}^{c} \leqslant G_{r}$.

Firstly, we replace $\tau_{\mathbf{I}_{\mathbf{j} r}}^{*}$ with $\tau_{\mathbf{I}_{l}}^{\approx}\left(\tau_{\mathbf{I}_{\mathbf{j}} r}^{*}\right)$. We may rewrite the roots of $\tau_{\mathbf{I}_{\mathbf{j} r}}^{*}, \tau_{\mathbf{I}_{l}}^{\approx /}\left(\tau_{\mathbf{I}_{\mathbf{j} r}}^{*}\right)$ as

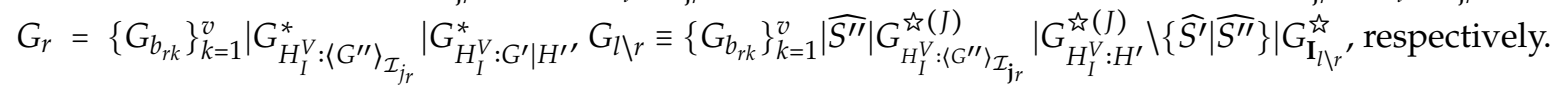

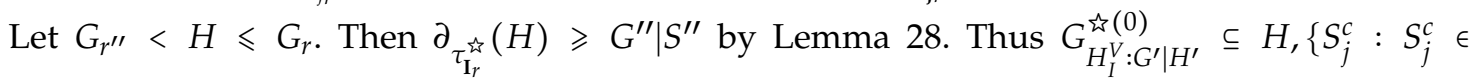
$\left.G_{H_{I}^{V}:\left\langle G^{\prime \prime}\right\rangle_{\mathcal{I}_{j_{r}}}}, H_{j}^{c} \geqslant G^{\prime \prime} \mid S^{\prime \prime}\right\}=\left\{S_{j}^{c}: S_{j}^{c} \in G_{H_{I}^{V}:\left\langle G^{\prime \prime}\right\rangle_{\mathcal{I}_{j r}}}^{\text {זे }(J)}, H_{j}^{c} \geqslant G^{\prime \prime} \mid S^{\prime \prime}\right\}$. Define $G_{H}^{* *}=\left\{S_{j}^{c}: S_{j}^{c} \in\right.$ $G_{H_{I}^{V}:\left\langle G^{\prime \prime}\right\rangle_{\mathcal{I}_{j_{r}}}}^{*}, S_{j}^{c}$ be the focus sequent of some $\left.H^{\prime} \in \tau_{\mathbf{I}_{r}: G_{q+1}^{\circ}}^{\mathfrak{i n}(q)}(2), H \leqslant H^{\prime} \leqslant G_{r}\right\}$.

Then we replace $H$ with

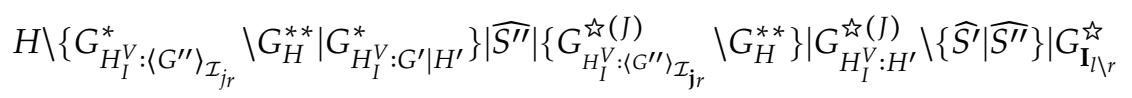

for all $G_{b_{r^{\prime} k^{\prime}}} \mid S_{j_{r^{\prime} k^{\prime}}}^{c} \leqslant H \leqslant G_{r}$. 


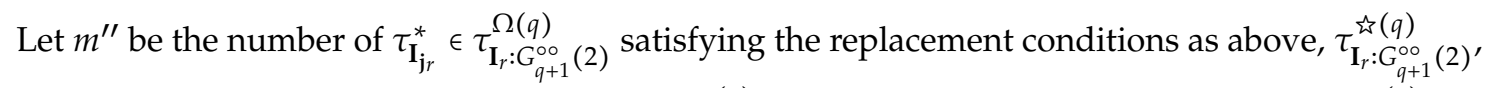

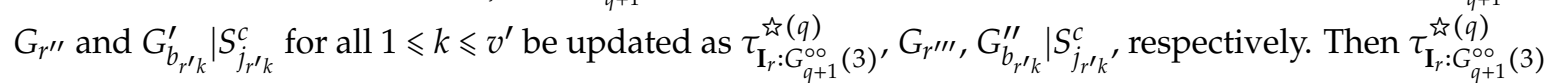
is a derivation and $G_{r^{\prime \prime \prime}}=G_{r^{\prime \prime}} \backslash H_{1}^{m^{\prime \prime}} \mid H_{2}^{m^{\prime \prime}}$, where

$$
\begin{aligned}
& H_{0}=G_{G_{r^{\prime} k}^{\prime}}^{* *} \mid S_{j_{r^{\prime} k}}^{c}, \\
& H_{1}=G_{H_{I}^{V}:\left\langle G^{\prime \prime}\right\rangle_{\mathcal{I}_{r}}} \backslash H_{0} \mid G_{H_{I}^{V}: G^{\prime} \mid H^{\prime \prime}}^{*}
\end{aligned}
$$

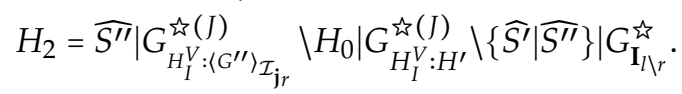

Step 4 (Separation along $H_{I}^{V}$ ). Apply the separation algorithm along $H_{I}^{V}$ to $G_{r^{\prime \prime \prime}}$ and denote the resulting derivation by $\tau_{\mathbf{I}_{r}: G_{q+1}^{\circ}}^{i(q)}(4)$ whose root is labeled by $G_{q+1}^{*}$.

By Claim (iii), $G_{H_{I}^{V}: G_{r^{\prime}}}^{\grave{\imath}(J)}=G_{q+1}^{\circ \circ}$.

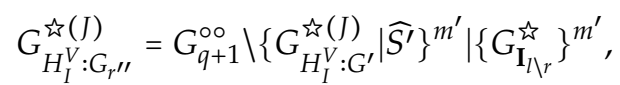

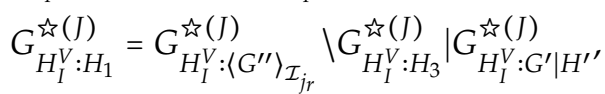

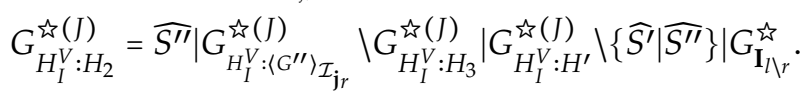

Then

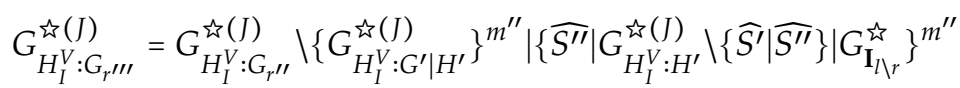

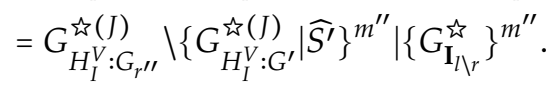

Then

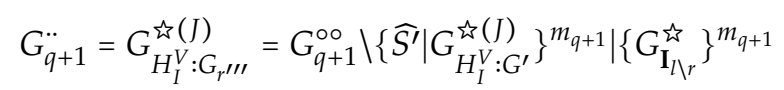

where $m_{q+1}:=m^{\prime}+m^{\prime \prime}$.

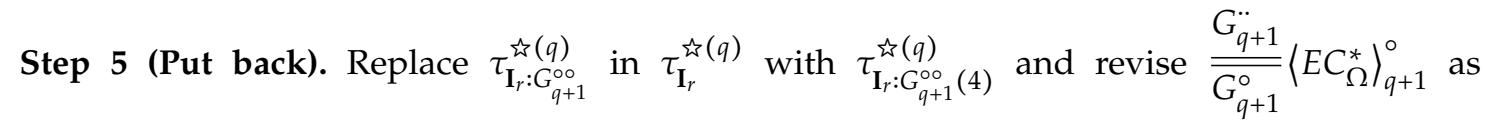
$\frac{G_{q+1}^{*}}{\overline{G_{q+1}^{\circ}}}\left\langle E C_{\Omega}^{*}\right\rangle_{q+1}^{.}$. Define the resulting tree from Step 5 to be $\tau_{\mathbf{I}_{r}}^{\imath(q+1)}$ then Claims (a), (b) and (c) hold for $q+1$ by the above construction.

Finally, we construct a derivation of $G_{\mathbf{I}_{r}}^{i j} \backslash\left\{\widehat{S^{\prime}} \mid G_{H_{I}^{V}: G^{\prime}}^{\hbar(J)}\right\} \mid G_{\mathbf{I}_{l \mid r}}^{\text {is }}$ from $\left\lceil S_{i_{1}}^{c}\right\rceil_{I}, \ldots,\left\lceil S_{i_{m}}^{c}\right\rceil_{I}$ in $\mathbf{G} \mathbf{L}_{\Omega}$. Since the

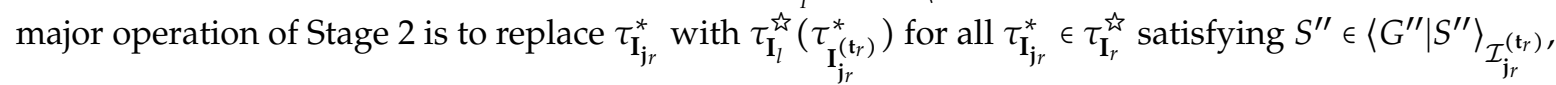
then we denote the resulting derivation from Stage 2 by $\tau_{\mathbf{I}_{r}}^{\hbar_{r}}\left(\tau_{\mathbf{I}_{l}}^{\boldsymbol{\nu}_{l}}\left(\tau_{\mathbf{I}_{r}}^{*}\right)\right)$.

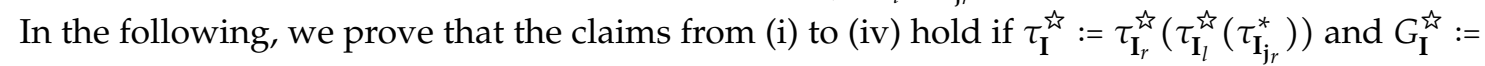

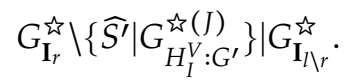

- For Claims (i) and (ii): Let $\frac{H_{1} \cdots H_{w}}{H_{0}}\left|\tau_{\mathbf{I}_{\mathbf{j}}}^{*}\right\rangle \in \tau_{\mathbf{I}}^{\grave{j}}$ and $S_{j}^{c} \in G_{\mathcal{I}_{\mathbf{j}}}^{*}$. Then $\partial_{\tau_{\mathbf{I}}^{\varkappa}}\left(H_{k}\right) \nless H_{j}^{c}$ for all $1 \leqslant k \leqslant w$ by Lemma 17 (iv). 


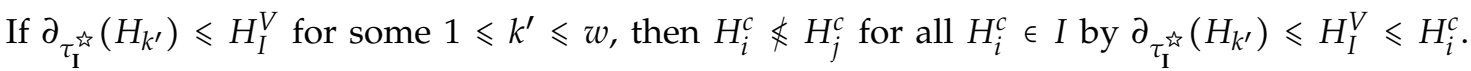
Thus Claim (i) holds and Claim (ii) holds by Lemma 25(5) and Lemma 19(i). Note that Lemma 25(5) is independent of Claims from (ii) to (iv).

Otherwise $\tau_{\mathbf{I}_{\mathbf{j}}}^{*}$ is built up from $\tau_{\mathbf{I}_{\mathbf{j}_{r}}}^{*} \in \tau_{\mathbf{I}_{r}}^{i \vec{j}}, \tau_{\mathbf{I}_{\mathbf{j}_{l}}}^{*}$ or $\tau_{\mathbf{I}_{\mathbf{j}_{l}} \cup \mathbf{I}_{\mathbf{j}_{r}}}^{*} \in \tau_{\mathbf{I}_{l}}^{\hbar_{l}}\left(\tau_{\mathbf{I}_{\mathbf{j}_{r}}}^{*}\right)$ by keeping their focus and principal sequents unchanged and making their side-hypersequents possibly to be modified, but which has no effect on discussing Claim (ii) and then Claim (ii) holds for $\tau_{\mathbf{I}}^{\tau^{2}}$ by the induction

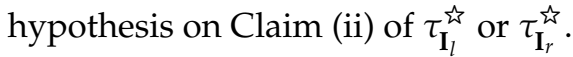

If $\tau_{\mathbf{I}_{\mathbf{j}}}^{*}$ is from $\tau_{\mathbf{I}_{\mathbf{j}_{l}} \cup \mathbf{I}_{\mathbf{j}_{r}}}^{*}$ then $S^{\prime} \in\left\langle G^{\prime} \mid S^{\prime}\right\rangle_{\mathcal{I}_{\mathbf{j}_{l}}}$ and $S^{\prime \prime} \in\left\langle G^{\prime \prime} \mid S^{\prime \prime}\right\rangle_{\mathcal{I}_{\mathbf{j}_{r}}}$ by the choice of $\tau_{\mathbf{I}_{\mathbf{j}_{l}}}^{*}$ and $\tau_{\mathbf{I}_{\mathbf{j}_{r}}}^{*}$ at Stage 1. By the induction hypothesis, $H_{i}^{c} \nless H_{j}^{c}$ for all $S_{j}^{c} \in G_{\mathcal{I}_{j_{l}}}^{*}, H_{i}^{c} \in I_{l}$ and $H_{i}^{c} \nless H_{j}^{c}$ for all $S_{j}^{c} \in G_{\mathcal{I}_{j_{r}}}^{*} H_{i}^{c} \in I_{r}$. Then $H_{i}^{c} \nless H_{j}^{c}$ for all $S_{j}^{c} \in G_{\mathcal{I}_{\mathbf{j}}}^{*}=G_{\mathcal{I}_{\mathfrak{j}_{l}} \cup \mathcal{I}_{\mathbf{j}_{r}}}^{*}, H_{i}^{c} \in I$ by $G_{\mathcal{I}_{\mathfrak{j}_{l}} \cup \mathcal{I}_{\mathbf{j}_{r}}}^{*}=G_{\mathcal{I}_{\mathfrak{j}_{l}}}^{*} \cap G_{\mathcal{I}_{\mathbf{j}_{r}}}^{*}, I=I_{l} \cup I_{r}$.

If $\tau_{\mathbf{I}_{\mathbf{j}}}^{*}$ is from $\tau_{\mathbf{I}_{\mathbf{j}_{l}}}^{*}$ then $S^{\prime} \notin\left\langle G^{\prime} \mid S^{\prime}\right\rangle_{\mathcal{I}_{\mathbf{j}_{l}}}$ by Step 3 at Stage 1. Then $\left\langle G^{\prime}\left|G^{\prime \prime}\right| H^{\prime}\right\rangle_{\mathcal{I}_{\mathbf{j}_{l}}} \cap\left(G^{\prime \prime} \mid H^{\prime}\right)=\varnothing$. Thus $S_{j}^{c} \notin G_{H_{I}^{V}: G^{\prime \prime} \mid H^{\prime}}^{*}$. Hence $G^{\prime \prime} \mid S^{\prime \prime} \nless H_{j}^{c}$. Therefore $H_{i}^{c} \nless H_{j}^{c}$ for all $H_{i}^{c} \in I_{r}$ by $G^{\prime \prime} \mid S^{\prime \prime} \leqslant H_{i}^{c}$. Thus $H_{i}^{c} \nless H_{j}^{c}$ for all $H_{i}^{c} \in I$ by $S_{j}^{c} \in G_{\mathcal{I}_{\mathbf{j}}}^{*}=G_{\mathcal{I}_{\mathbf{j}_{l}}}^{*}$ and the induction hypothesis from $\tau_{\mathbf{I}_{\mathbf{j}_{l}}}^{*} \in \tau_{\mathbf{I}_{l}}^{\tilde{I}_{j}}$. The case of $\tau_{\mathbf{I}_{\mathbf{j}}}^{*}$ built up from $\tau_{\mathbf{I}_{\mathbf{j}_{r}}}^{*}$ is proved by a procedure similar to above and omitted.

- Claim (iii) holds by Step 4 at Stages 1 and 2. Note that in the whole of Stage 1, we treat $\left\{G_{b_{r k}}\right\}_{k=1}^{v}$ as a side-hypersequent. But it is possible that there exists $S_{j}^{c} \in\left\{G_{b_{r k}}\right\}_{k=1}^{v}$ such that $H_{j}^{c} \leqslant H_{I}^{V}$. Since we have not applied the separation algorithm to $\left\{G_{b_{r k}}\right\}_{k=1}^{v}$ in Step 4 at Stage 1, then it could make Claim (iii) invalid. But it is not difficult to find that we just move the separation of such $S_{j}^{c}$ to Step 4 at Stage 2. Of course, we can move it to Step 4 at Stage 1, but which make the discussion complicated.

- For Claim (iv), we prove (1) $H_{i}^{c} \| H_{j}^{c}$ for all $S_{j}^{c} \in G_{\mathbf{I}_{l \backslash r}}^{\text {is }}$ and $H_{i}^{c} \in I$, (2) $H_{i}^{c} \| H_{j}^{c}$ for all $S_{j}^{c} \epsilon$ $G_{\mathbf{I}_{r}}^{i j} \backslash\left\{\widehat{S^{\prime}} \mid G_{H_{I}^{V}: G^{\prime}}^{i(J)}\right\}$ and $H_{i}^{c} \in I$. Only (1) is proved as follows and (2) by a similar procedure and omitted.

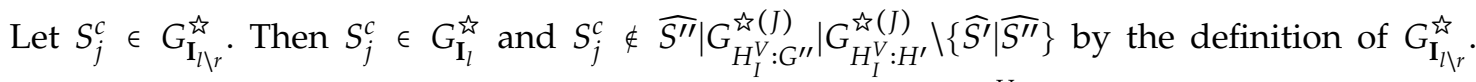
By a procedure similar to that of Claim (iv) in Case 1, we get $H_{j}^{c} \nless H_{I}^{V}$ and assume that $S_{j}^{c} \in G_{\mathcal{I}_{j_{l}}}^{*}$ for some $\tau_{\mathbf{I}_{\mathbf{j}_{l}}}^{*} \in \tau_{\mathbf{I}_{l}}^{i}$ and let $G^{\prime} \mid S^{\prime} \nless H_{j}^{c}$ in the following.

Suppose that $G^{\prime \prime} \mid S^{\prime \prime} \leqslant H_{j}^{c}$. Then $S_{j}^{c} \in G_{H_{I}^{V}: G^{\prime \prime}}^{*}$ and $S^{\prime} \in\left\langle G^{\prime} \mid S^{\prime}\right\rangle_{\mathcal{I}_{\mathfrak{j}_{l}}}$ by $S_{j}^{c} \in G_{\mathcal{I}_{\mathfrak{j}_{l}}}^{*}$. Hence $S_{j}^{c} \in G_{H_{I}^{V}: G^{\prime \prime}}^{\mathfrak{i n}(J)}$

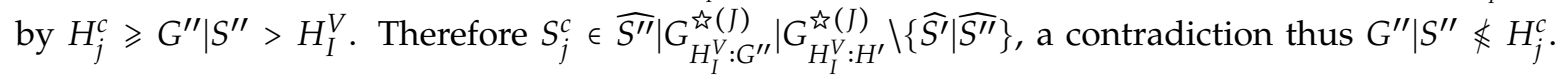
Then $H_{I}^{V} \nless H_{j}^{c}$ by $G^{\prime} \mid S^{\prime} \nless H_{j}^{c}$ and $G^{\prime \prime} \mid S^{\prime \prime} \nless H_{j}^{c}$. Thus $H_{j}^{c} \| H_{I}^{V}$. Hence $H_{j}^{c} \| H_{i}^{c}$ for all $H_{i}^{c} \in I$. This completes the proof of Theorem 2.

Definition 31. The manipulation described in Theorem 2 is called a derivation-grafting operation.

\section{The Proof of the Main Theorem}

Recall that in the main theorem $G_{0} \equiv G^{\prime}\left|\left\{\Gamma_{i}, p \Rightarrow \Delta_{i}\right\}_{i=1 \cdots n}\right|\left\{\Pi_{j} \Rightarrow p, \Sigma_{j}\right\}_{j=1 \cdots m}$.

Lemma 29. (i) If $G_{2}=G_{0} \backslash\left\{\Gamma_{1}, p \Rightarrow \Delta_{1}\right\}$ and $\vdash_{\mathrm{GL}} \mathcal{D}_{0}\left(G_{2}\right)$ then $\vdash_{\mathrm{GL}} \mathcal{D}_{0}\left(G_{0}\right)$;

(i') If $G_{2}=G_{0} \backslash\left\{\Pi_{1} \Rightarrow p, \Sigma_{1}\right\}$ and $\vdash_{\mathrm{GL}} \mathcal{D}_{0}\left(G_{2}\right)$ then $\vdash_{\mathrm{GL}} \mathcal{D}_{0}\left(G_{0}\right)$;

(ii) If $G_{2}=G_{0} \mid\left\{\Gamma_{1}, p \Rightarrow \Delta_{1}\right\}$ and $\vdash_{\mathrm{GL}} \mathcal{D}_{0}\left(G_{2}\right)$ then $\vdash_{\mathrm{GL}} \mathcal{D}_{0}\left(G_{0}\right)$;

(ii') If $G_{2}=G_{0} \mid\left\{\Pi_{1} \Rightarrow p, \Sigma_{1}\right\}$ and $\vdash_{\mathrm{GL}} \mathcal{D}_{0}\left(G_{2}\right)$ then $\vdash_{\mathrm{GL}} \mathcal{D}_{0}\left(G_{0}\right)$;

(iii) If $G_{2}=G_{0} \backslash\left\{\Gamma_{1}, p \Rightarrow \Delta_{1}\right\} \mid\left\{\Gamma_{1}, T \Rightarrow \Delta_{1}\right\}$ and $\vdash_{\mathrm{GL}} \mathcal{D}_{0}\left(G_{2}\right)$ then $\vdash_{\mathrm{GL}} \mathcal{D}_{0}\left(G_{0}\right)$;

(iii') If $G_{2}=G_{0} \backslash \Pi_{1} \Rightarrow p, \Sigma_{1} \mid \Pi_{1} \Rightarrow \perp, \Sigma_{1}$ and $\vdash_{\mathrm{GL}} \mathcal{D}_{0}\left(G_{2}\right)$ then $\vdash_{\mathrm{GL}} \mathcal{D}_{0}\left(G_{0}\right)$. 
Proof. (i) Since $\mathcal{D}_{0}\left(G_{2}\right)=G^{\prime}\left|\left\{\Gamma_{i}, \Pi_{j} \Rightarrow \Delta_{i}, \Sigma_{j}\right\}_{i=2 \cdots n ; j=1 \cdots m} \subseteq G^{\prime}\right|\left\{\Gamma_{1}, \Pi_{j} \Rightarrow \Delta_{1}, \Sigma_{j}\right\}_{j=1 \cdots m} \mid\left\{\Gamma_{i}, \Pi_{j} \Rightarrow\right.$ $\left.\Delta_{i}, \Sigma_{j}\right\}_{i=2 \cdots n ; j=1 \cdots m}=\mathcal{D}_{0}\left(G_{0}\right)$ then $\vdash_{\mathrm{GL}} \mathcal{D}_{0}\left(G_{0}\right)$ holds. If $n=1$, we replace all $p$ in $\Pi_{j} \Rightarrow p, \Sigma_{j}$ with $\perp$. Then $\vdash_{\mathrm{GL}} \mathcal{D}_{0}\left(G_{0}\right)$ holds by applying $(C U T)$ to $\Gamma_{1}, \perp \Rightarrow \Delta_{1}$ and $G^{\prime} \mid\left\{\Pi_{j} \Rightarrow \perp, \Sigma_{j}\right\}_{j=1 \cdots m}$.

(ii) Since $\mathcal{D}_{0}\left(G_{2}\right)=G^{\prime}\left|\left\{\Gamma_{1}, \Pi_{j} \Rightarrow \Delta_{1}, \Sigma_{j}\right\}_{j=1 \cdots m}\right|\left\{\Gamma_{i}, \Pi_{j} \Rightarrow \Delta_{i}, \Sigma_{j}\right\}_{i=1 \cdots n ; j=1 \cdots m}$ then $\vdash_{\mathrm{GL}} \mathcal{D}_{0}\left(G_{0}\right)$ holds by applying $\left(E C^{*}\right)$ to $\mathcal{D}_{0}\left(G_{2}\right)$.

(iii) Since $\mathcal{D}_{0}\left(G_{2}\right)=G^{\prime}\left|\Gamma_{1}, \mathrm{~T} \Rightarrow \Delta_{1}\right|\left\{\Gamma_{i}, \Pi_{j} \Rightarrow \Delta_{i}, \Sigma_{j}\right\}_{i=2 \cdots n ; j=1 \cdots m}$ then $\vdash_{\mathrm{GL}} G^{\prime \prime} \equiv G^{\prime} \mid \Gamma_{1}, \Pi_{1} \Rightarrow$ $\Delta_{1}, \Sigma_{1} \mid\left\{\Gamma_{i}, \Pi_{j} \Rightarrow \Delta_{i}, \Sigma_{j}\right\}_{i=2 \cdots n ; j=1 \cdots m}$ holds by applying $(C U T)$ to $\Gamma_{1}, T \Rightarrow \Delta_{1}$ in $\mathcal{D}_{0}\left(G_{2}\right)$ and $\Pi_{1} \Rightarrow \mathrm{T}, \Sigma_{1}$. Thus $\vdash_{\mathrm{GL}} \mathcal{D}_{0}\left(G_{0}\right)$ holds by applying $(E W)$ to $G^{\prime \prime}$.

$\left(\mathrm{i}^{\prime}\right),\left(\mathrm{ii}^{\prime}\right)$ and (iii') are proved by a procedure respectively similar to those of (i), (ii) and (iii) and omitted.

Let $I=\left\{H_{i_{1}}^{c}, \cdots, H_{i_{m}}^{c}\right\} \subseteq\left\{H_{1}^{c}, \cdots, H_{N}^{c}\right\}, G_{I}$ denote a closed hypersequent such that $G_{I} \subseteq_{c} G \mid G^{*}$ and $H_{j}^{c} \| H_{i}^{c}$ for all $S_{j}^{c} \in G_{I}$ and $H_{i}^{c} \in I$.

Lemma 30. There exists $G_{I}$ such that $\vdash_{\mathbf{G L}_{\Omega}} G_{I}$ for all $I \subseteq\left\{H_{1}^{c}, \cdots, H_{N}^{c}\right\}$.

Proof. The proof is by induction on $m$. For the base step, let $m=0$, then $I=\varnothing$ and $G_{I}:=G \mid G^{*}$ and $\vdash_{\mathrm{GL}_{\Omega}} G_{I}$ by Lemma $5(\mathrm{v})$.

For the induction step, suppose that $m \geqslant 1$ and there exists $G_{I}$ such that $\vdash_{\mathbf{G L}_{\Omega}} G_{I}$ for all $|I| \leqslant m-1$. Then there exist $G_{I \backslash\left\{H_{i_{k}}^{c}\right.}$ for all $1 \leqslant k \leqslant m$ such that $\vdash_{\mathbf{G L}_{\Omega}} G_{I \backslash\left\{H_{i_{k}}^{c}\right\}}$ and $H_{j}^{c} \| H_{i}^{c}$ for all $S_{j}^{c} \in G_{I \backslash\left\{H_{i_{k}}^{c}\right\}}$ and $H_{i}^{c} \in I \backslash\left\{H_{i_{k}}^{c}\right\}$.

If $H_{j}^{c} \| H_{i_{k}}^{c}$ for all $S_{j}^{c} \in G_{I \backslash\left\{H_{i_{k}}^{c}\right\}}$ then $G_{I}:=G_{I \backslash\left\{H_{i_{k}}^{c}\right\}}$ and the claim holds clearly. Otherwise there exists $S_{j}^{c} \in G_{I \backslash\left\{H_{i_{k}}^{c}\right\}}$ such that $H_{j}^{c} \leqslant H_{i_{k}}^{c}$ or $H_{j}^{c}>H_{i_{k}}^{c}$ then we rewrite $G_{I \backslash\left\{H_{i_{k}}^{c}\right\}}$ as $\left\lceil S_{i_{k}^{\prime}}^{c}\right]_{\left\{H_{i_{k}^{\prime}}^{c}\right\} \cup I \backslash\left\{H_{i_{k}}^{c}\right\}}$, where we define $H_{i_{k}^{\prime}}^{c}$ such that $S_{i_{k}^{\prime}}^{c} \in G_{I \backslash\left\{H_{i_{k}}^{c}\right\}}$ and, $S_{j}^{c} \in G_{I \backslash\left\{H_{i_{k}}^{c}\right\}}$ implies $H_{j}^{c} \leqslant H_{i_{k}^{\prime}}^{c}$ or $H_{j}^{c} \| H_{i}^{c}$ for all $H_{i}^{c} \in\left\{H_{i_{k}^{\prime}}^{c}\right\} \cup I \backslash\left\{H_{i_{k}}^{c}\right\}$. If we cannot define $G_{I}$ to be $G_{I \backslash\left\{H_{i_{k}}^{c}\right\}}$ for each $1 \leqslant k \leqslant m$, let $I^{\prime}:=\left\{H_{i_{1}^{\prime}}^{c}, \cdots, H_{i_{m}^{\prime}}^{c}\right\}$. Then $G_{I^{\prime}}$ is constructed by applying the separation algorithm of multiple branches (or one branch if $m=1$ ) to $\left\lceil S_{i_{1}^{\prime}}^{c}\right\rceil_{I^{\prime}}, \cdots,\left\lceil S_{i_{m}^{\prime}}^{c}\right\rceil_{I^{\prime}}$. Then $\vdash \mathbf{G L}_{\Omega} G_{I^{\prime}}$ by $\vdash \mathbf{G L}_{\Omega}\left\lceil S_{i_{1}^{\prime}}^{c}\right]_{I^{\prime}}, \cdots, \vdash \mathbf{G L}_{\Omega}\left\lceil S_{i_{m}^{\prime}}^{c}\right\rceil_{I^{\prime}}$, Theorem 2 (or Lemma 20(i) for one branch). Let $G_{I}:=G_{I^{\prime}}$ then $\vdash \mathbf{G L}_{\Omega} G_{I}$ clearly.

The proof of Theorem 1: Let $I=\left\{H_{1}^{c}, \cdots, H_{N}^{c}\right\}$ in Lemma 30. Then there exists $G_{I}$ such that $\vdash_{\mathbf{G L}_{\Omega}} G_{I}$, $G_{I} \subseteq_{c} G \mid G^{*}$ and $H_{j}^{c} \| H_{i}^{c}$ for all $S_{j}^{c} \in G_{I}$ and $H_{i}^{c} \in I$. Then $\vdash_{\mathrm{GL}} \mathcal{D}\left(G_{I}\right)$ by Lemma 8 .

Suppose that $S_{j}^{c} \in G_{I}$. Then $H_{j}^{c} \| H_{i}^{c}$ for all $H_{i}^{c} \in I$. Thus $H_{j}^{c} \| H_{j}^{c}$ by $H_{j}^{c} \in I$, a contradiction with $H_{j}^{c} \leqslant H_{j}^{c}$ and hence there does not exist $S_{j}^{c} \in G_{I}$. Therefore $G_{I} \subseteq_{c} G$ by $G_{I} \subseteq_{c} G \mid G^{*}$.

By removing the identification number of each occurrence of $p$ in $G$, we obtain the sub-hypersequent $G_{2}$ of $G_{2} \mid G_{2}^{*}$, which is the root of $\tau^{4}$ resulting from Step 4 in Section 4 . Then $\vdash_{\text {GL }}$ $\mathcal{D}_{0}\left(G_{2}\right)$ by $\vdash_{\mathrm{GL}} \mathcal{D}\left(G_{I}\right)$ and $G_{I} \subseteq_{c} G$. Since $G_{2}$ is constructed by adding or removing some $\Gamma_{i}, p \Rightarrow \Delta_{i}$ or $\Pi_{j} \Rightarrow p, \Sigma_{j}$ from $G_{0}$, or replacing $\Gamma_{i}, p \Rightarrow \Delta_{i}$ with $\Gamma_{i}, \top \Rightarrow \Delta_{i}$, or $\Pi_{j} \Rightarrow p, \Sigma_{j}$ with $\Pi_{j} \Rightarrow \perp, \Sigma_{j}$, then $\vdash_{\mathrm{GL}} \mathcal{D}_{0}\left(G_{0}\right)$ by Lemma 29 . This completes the proof of the main theorem.

Theorem 3. Density elimination holds for all GL in $\{$ GUL, GIUL, GMTL, GIMTL $\}$.

Proof. It follows immediately from the main theorem.

\section{Final Remarks and Open Problems}

Recently, we have generalized our method described in this paper to the non-commutative substructural logic GpsUL $^{*}$ in [20]. This result shows that GpsUL $^{*}$ is the logic of pseudo-uninorms and their residua and answered the question posed by Metcalfe, Olivetti, Gabbay and Tsinakis in $[21,22]$.

It has often been the case in the past that metamathematical proofs of the standard completeness have the corresponding algebraic ones, and vise verse. In particular, Baldi and Terui [23] had given 
an algebraic proof of the standard completeness of UL. A natural problem is whether there is an algebraic proof corresponding to our proof-theoretic one. It seems difficult to obtain it by using the insights gained from the approach described in this paper because ideas and syntactic manipulations introduced here are complicated and specialized. In addition, Baldi and Terui [23] also mentioned some open problems. Whether our method could be applied to their problems is another research direction.

On 21 March 2014, I found the way to deal with the example in Section 3. Then I finished the one branch algorithm in Section 7 on the late April 2014. I devised the multi-branch algorithm in Section 8 on early November 2014. Since I submitted my paper to Transactions of the American Mathematical Society on 20 January 2015, it has been reviewed successively by Annals of Pure and Applied Logic, Fuzzy Sets and Systems and, the Journal of Logic and Computation. As a mathematician, the greatest anxiety is that his work has never been taken seriously by his academic circle during his career, but after his death, someone would say, sir, your proof is wrong.

Funding: This research was funded by the National Foundation of Natural Sciences of China (Grant No: 61379018 $\& 61662044 \& 11571013 \& 11671358)$.

Acknowledgments: I am grateful to Lluis Godo, Arnon Avron, Jean-Yves Girard, George Metcalfe and Agata Ciabattoni for valuable discussions. I would like to thank anonymous reviewers for carefully reading the old version of this article and many instructive suggestions.

Conflicts of Interest: The authors declare no conflict of interest.

\section{Notations}

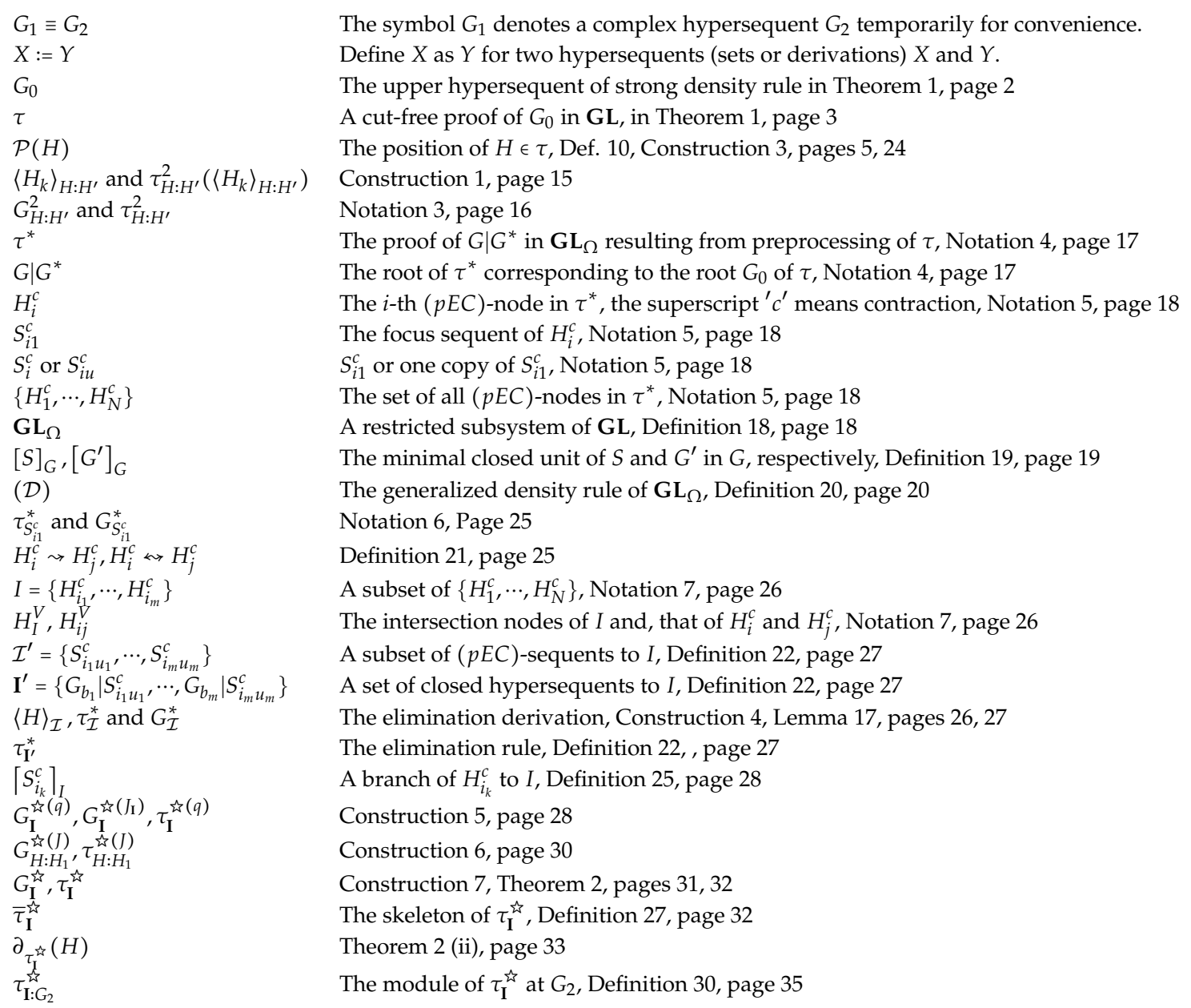




\section{Appendix A.}

Appendix A.1. Why Do We Adopt Avron-Style Hypersequent Calculi?

A hypersequent calculus is called Pottinger-style if its two-premise rules are in the form of $\frac{G\left|S^{\prime} G\right| S^{\prime \prime}}{G \mid H^{\prime}}(I I)$ and, Avron-style if in the form of $\frac{G^{\prime}\left|S^{\prime} G^{\prime \prime}\right| S^{\prime \prime}}{G^{\prime}\left|G^{\prime \prime}\right| H^{\prime}}(I I)$. In the viewpoint of Avron-style systems, each application of two-premise rules contains implicitly applications of $(E C)$ in Pottinger-style systems, as shown in the following.

$$
\frac{G\left|S^{\prime} \quad G\right| S^{\prime \prime}}{G \mid H^{\prime}}(I I) \underset{\text { in Avron-style system }}{\text { corresponds to }} \frac{\frac{G\left|S^{\prime} G\right| S^{\prime \prime}}{G|G| H^{\prime}}(I I)}{G \mid H^{\prime}}\left(E C^{*}\right)
$$

The choice of the underlying system of hypersequent calculus is vital to our purpose and it gives the background or arena. In Pottinger-style system, $G_{0}$ in Section 3 is proved without application of $(E C)$ as follows. But it seems helpless to prove that $H_{0}$ is a theorem of IUL.

\begin{tabular}{|c|c|c|}
\hline \multirow[b]{4}{*}{$C \Rightarrow C$} & \multirow[b]{3}{*}{$B \Rightarrow B$} & $\frac{p \Rightarrow p \quad A \Rightarrow A}{A \Rightarrow p \mid p \Rightarrow A} \frac{p \Rightarrow p \quad A \Rightarrow A}{A \Rightarrow p \mid p \Rightarrow A} \frac{p \Rightarrow p \quad A \Rightarrow A}{A \Rightarrow p \mid p \Rightarrow A} \frac{p \Rightarrow p \quad A \Rightarrow A}{A \Rightarrow p \mid p \Rightarrow A}$ \\
\hline & & $A \Rightarrow p|p, p \Rightarrow A \odot A \quad A \Rightarrow p| p, p \Rightarrow A \odot A$ \\
\hline & & $\Rightarrow p, \neg A \mid p, p \Rightarrow A \odot A$ \\
\hline & $\overline{B \Rightarrow B \mid p, p \Rightarrow A \odot A}$ & $\Rightarrow p, p, \neg A \odot \neg A \mid p, p \Rightarrow A \odot A$ \\
\hline$C \Rightarrow C|\Rightarrow p, B| B \Rightarrow p, \neg A \odot \neg A$ & \multicolumn{2}{|r|}{$\Rightarrow p, B|B \Rightarrow p, \neg A \odot \neg A| p, p \Rightarrow A \odot A$} \\
\hline \multicolumn{3}{|c|}{$\Rightarrow p, B|B \Rightarrow p, \neg A \odot \neg A| p \Rightarrow C \mid C, p \Rightarrow A \odot A$} \\
\hline
\end{tabular}

The peculiarity of our method is not only to focus on controlling the role of the external contraction rule in the hypersequent calculus but also introduce other syntactic manipulations. For example, we label occurrences of the eigenvariable $p$ introduced by an application of the density rule in order to be able to trace these occurrences from the leaves (axioms) of the derivation to the root (the derived hypersequent).

Appendix A.2. Why Do We Need the Constrained External Contraction Rule?

We use the example in Section 3 to answer this question. Firstly, we illustrate Notation 5 as follows. In Figure 4, let $S_{11}^{c}=A \Rightarrow p_{2} ; S_{12}^{c}=A \Rightarrow p_{1} ; S_{21}^{c}=A \Rightarrow p_{4} ; S_{22}^{c}=A \Rightarrow p_{3} ; S_{31}^{c}=p_{1}, p_{2} \Rightarrow A \odot A$; $S_{32}^{c}=p_{3}, p_{4} \Rightarrow A \odot A ; G_{1}^{\prime}=p_{1}, p_{2} \Rightarrow A \odot A ; G_{2}^{\prime}=p_{3}, p_{4} \Rightarrow A \odot A ; G_{3}^{\prime}=A \Rightarrow p_{1}\left|\Rightarrow p_{2}, B\right| B \Rightarrow p_{4}, \neg A \odot$ $\neg A \mid A \Rightarrow p_{3}$. Then $H_{i}^{c}=G_{i}^{\prime}\left|S_{i 1}^{c}\right| S_{i 2}^{c}$ for $i=1,2,3$. $H_{i}^{c}$ are $(p E C)$-nodes and, $S_{i 1}^{c}$ and $S_{i 2}^{c}$ are $(p E C)$-sequents.

Let $G_{H_{1}^{c}: A \Rightarrow p_{2}}^{*} \Rightarrow p_{2}, B\left|B \Rightarrow p_{4}, \neg A \odot \neg A\right| A \Rightarrow p_{3} \mid p_{3}, p_{4} \Rightarrow A \odot A$. We denote the derivation $\tau_{H_{1}^{c}: A \Rightarrow p_{2}}^{*}$ of $G_{H_{1}^{c}: A \Rightarrow p_{2}}^{*}$ from $A \Rightarrow p_{2}$ by $\frac{A \Rightarrow p_{2}}{\overline{G_{H_{1}^{c}: A \Rightarrow p_{2}}^{*}}}\left\langle\tau_{H_{1}^{c}: A \Rightarrow p_{2}}^{*}\right\rangle$. Since we focus on sequents in $G^{*}$ in the separation algorithm, we abbreviate $\frac{A \Rightarrow p_{2}}{\overline{G_{H_{1}^{c}: A \Rightarrow p_{2}}^{*}}}\left|\tau_{H_{1}^{c}: A \Rightarrow p_{2}}^{*}\right\rangle$ to $\frac{S_{11}^{c}}{\overline{S_{22}^{c} \mid S_{32}^{c}}}\left\langle\tau_{S_{11}^{c}}^{*}\right\rangle$ and further to $\frac{1}{2 \mid 3}\left\langle\tau_{1}^{*}\right\rangle$. Then the separation algorithm $\tau_{H_{1}^{c}: G \mid G^{*}}^{i \zeta}$ is abbreviated as

$$
\frac{\frac{\frac{1|2| 3}{2^{\prime}\left|3^{\prime}\right| 2 \mid 3}\left\langle\tau_{1}^{*}\right\rangle}{2^{\prime} \mid 2}\left\langle\tau_{3}^{*}, \tau_{3^{\prime}}^{*}\right\rangle}{2}\left\langle E C_{\Omega}\right\rangle
$$


where $2^{\prime}$ and $3^{\prime}$ are abbreviations of $A \Rightarrow p_{5}$ and $p_{5}, p_{6} \Rightarrow A \odot A$, respectively. We also write $2^{\prime}$ and $3^{\prime}$ respectively as 2 and 3 for simplicity. Then the whole separation derivation is given as follows.

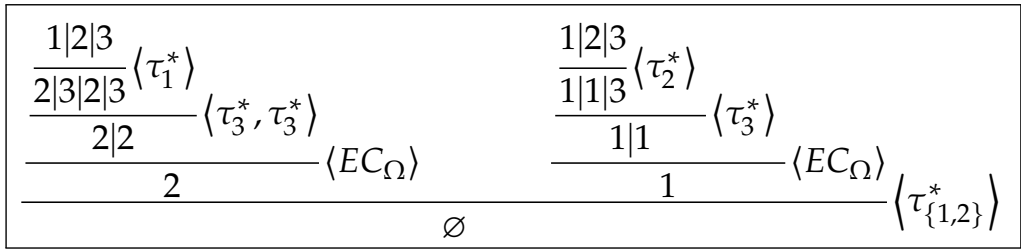

where $\varnothing$ is an abbreviation of $G^{\prime \prime}$ in page 14 and means that all sequents in it are copies of sequents in $G_{0}$. Note that the simplified notations become intractable when we decide whether $\left\langle E C_{\Omega}\right\rangle$ is applicable to resulting hypersequents. If no application of $\left\langle E C_{\Omega}\right\rangle$ is used in it, all resulting hypersequents fall into the set $\{1|2| 3|\cdots| 3,2|2| 3|\cdots| 3,1|1| 3|\cdots| 3: l \geq 0, m \geq 0, n \geq 0\}$ and $\varnothing$ is never obtained.

$$
\underbrace{3 \mid \cdots}_{l} \underbrace{3|\cdots|}_{m}, \underbrace{3|\cdots|}_{n}
$$

\section{Appendix A.3. Why Do We Need the Separation of Branches?}

In Figure 11, $p_{1}$ and $p_{2}$ in the premise of $\frac{p_{1}, p_{2} \Rightarrow A \odot A}{p_{1} \Rightarrow C \mid C, p_{2} \Rightarrow A \odot A}\left|\tau_{S_{31}^{c}}^{*}\right\rangle$ could be viewed as being tangled in one sequent $p_{1}, p_{2} \Rightarrow A \odot A$ but in the conclusion of $\left\langle\tau_{S_{11}^{c}}^{*}\right\rangle$ they are separated into two sequents $p_{1} \Rightarrow C$ and $C, p_{2} \Rightarrow A \odot A$, which are copies of sequents in $G_{0}$. In Figure $5, p_{2}$ in $A \Rightarrow p_{2}$ falls into $\Rightarrow p_{2}, B$ in the root of $\tau_{H_{1}^{c}: A \Rightarrow p_{2}}^{*}$ and $\Rightarrow p_{2}, B$ is a copy of a sequent in $G_{0}$. The same is true for $p_{4}$ in $A \Rightarrow p_{4}$ in Figure 8. But it's not the case.

Lemma 13(vi) shows that in the elimination rule $\frac{S_{11}^{c}}{\overline{G_{S_{11}^{c}}^{*}}}\left\langle\tau_{S_{11}^{c}}^{*}\right\rangle, S_{j}^{c} \in G_{S_{11}^{c}}^{*}$ implies $H_{j}^{c}<H_{i}^{c}$ or $H_{j}^{c} \| H_{i}^{c}$. If there exists no $S_{j}^{c} \in G_{S_{11}^{c}}^{*}$ such that $H_{j}^{c}<H_{i}^{c}$, then $S_{j}^{c} \in G_{S_{11}^{c}}^{*}$ implies $H_{j}^{c} \| H_{i}^{c}$ and, thus each occurrence of $p^{\prime} s$ in $S_{11}^{c}$ is fell into a unique sequent which is a copy of a sequent in $G_{0}$. Otherwise there exists $S_{j}^{c} \in G_{S_{11}^{c}}^{*}$ such that $H_{j}^{c}<H_{i}^{c}$, then we apply $\left\langle\tau_{S_{j}^{c}}^{*}\right\rangle$ to $S_{j}^{c}$ in $G_{S_{11}^{c}}^{*}$ and the whole operations can be written as

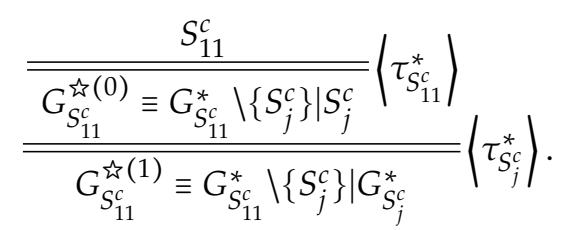

Repeatedly we can get $G_{S_{11}^{c}}^{i(J)}$ such that $S_{j}^{c} \in G_{S_{11}^{c}}^{i(J)}$ implies $H_{j}^{c} \| H_{1}^{c}$. Then each occurrence of $p^{\prime} s$ in $S_{11}^{c}$ is fell into a unique sequent in $G_{S_{11}^{c}}^{i(J)}$ which is a copy of a sequent in $G_{0}$. In such case, we call occurrences of $p^{\prime} s$ in $S_{11}^{c}$ are separated in $G_{S_{11}^{c}}^{i(J)}$ and call such a procedure the separation algorithm. It is the starting point of the separation algorithm. We introduce branches in order to tackle the case of multiple-premise separation derivations for which it is necessary to apply $\left(E C_{\Omega}\right)$ to the resulting hypersequents.

\section{Appendix A.4. Some Questions about Theorem 2}

In Theorem 2, $\tau_{I}^{\text {方 }}$ is constructed by induction on the number $|I|$ of branches. As usual, we take the algorithm of $|I|-1$ branches as the induction hypothesis. Why do we take $\tau_{\mathbf{I}_{l}}^{\boldsymbol{i n}^{3}}$ and $\tau_{\mathbf{I}_{r}}^{\hat{\hbar n}}$ as the induction hypothesises?

Roughly speaking, it degenerates the case of $|I|$ branches into the case of two branches in the following sense. The subtree $\tau^{*}\left(G^{\prime \prime} \mid S^{\prime \prime}\right)$ of $\tau^{*}$ is as a whole contained in $\tau_{\mathcal{I}_{j_{l}}}^{*}$ or not in it. Similarly, 
$\tau^{*}\left(G^{\prime} \mid S^{\prime}\right)$ of $\tau^{*}$ is as a whole contained in $\tau_{\mathcal{I}_{\mathbf{j} r}}^{*}$ or not in it. It is such a division of $I$ into $I_{l}$ and $I_{r}$ that makes the whole algorithm possible.

Claim (i) of Theorem 2 asserts that $H_{i}^{c} \nless H_{j}^{c}$ for all $S_{j}^{c} \in G_{\mathcal{I}_{\mathrm{j}}}^{*}$ and $H_{i}^{c} \in I$. It guarantees that $\tau_{\mathcal{I}_{\mathrm{j}}}^{*}$ is not far from the final aim of Theorem 2 but roughly close to it if we define some complexity to calculate it. If $H_{i}^{c} \leqslant H_{j}^{c}$, the complexity of $G_{\mathbf{I}_{\mathbf{j}}}^{*}$ is more than or equal to that of $\left[S_{i}^{c}\right\rceil_{I}$ under such a definition of complexity and thus such an application of $\tau_{\mathcal{I}_{j}}^{*}$ is redundant at least. Claim (iii) of Theorem 2 guarantees the validity of the step 4 of Stages 1 and 2 .

The tree structure of the skeleton of $\tau_{\mathbf{I}_{l}}^{\boldsymbol{i n}_{l}}\left(\tau_{\mathbf{I}_{\mathbf{j}_{r}}}^{*}\right)$ can be obtained by deleting some node $H \in \bar{\tau}_{\mathbf{I}_{l}}^{\hat{i}_{j}}$

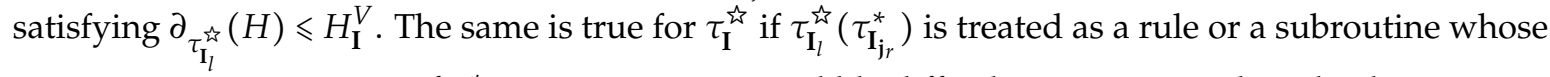
premises are same as ones of $\tau_{\mathbf{I}_{j}}^{*}$. However, it is incredibly difficult to imagine or describe the structure

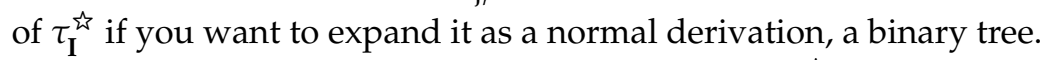

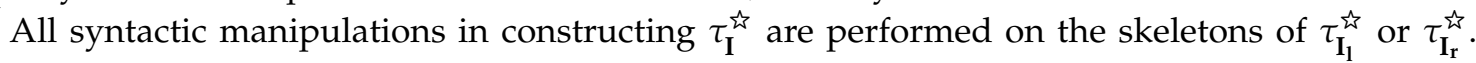
The structure of the proof of Theorem 2 is depicted in Figure A1.

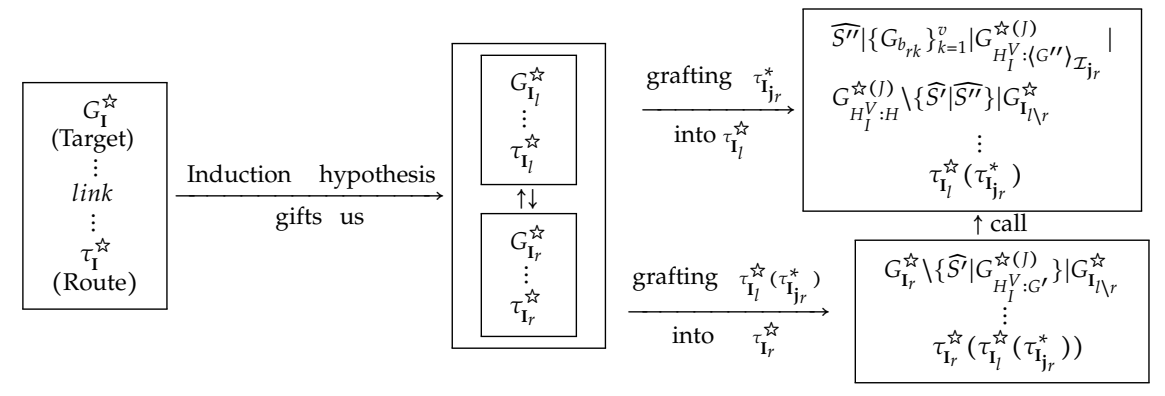

Figure A1. The structure of the proof of Theorem 2.

Appendix A.5. Illustrations of Notations and Algorithms

We use the example in Section 3 to illustrate some notations and algorithms in this paper.

Appendix A.5.1. Illustration of Two Cases of (COM) in the Proof of Lemma 8

Let $\frac{G^{\prime} G^{\prime \prime}}{G^{\prime \prime \prime}}(C O M)$ be $\frac{p_{1} \Rightarrow p_{1} \quad A \Rightarrow A}{A \Rightarrow p_{1} \mid p_{1} \Rightarrow A}(C O M)$, where $G^{\prime}=S_{1}=p_{1} \Rightarrow p_{1} ; G^{\prime \prime}=S_{2}=A \Rightarrow$ $A ; S_{3}=A \Rightarrow p_{1} ; S_{4}=p_{1} \Rightarrow A$ and $G^{\prime \prime \prime}=S_{3} \mid S_{4}$. Then $\left[S_{3}\right]_{G^{\prime \prime \prime}}=\left[S_{4}\right]_{G^{\prime \prime \prime}} ; \mathcal{D}_{G^{\prime}}\left(S_{1}\right)=\Rightarrow t$; $\mathcal{D}_{G^{\prime \prime}}\left(S_{2}\right)=A \Rightarrow A ; \mathcal{D}_{G^{\prime \prime \prime}}\left(S_{3} \mid S_{4}\right)=A \Rightarrow A$. Thus the proof of $\frac{\mathcal{D}_{G^{\prime}}\left(S_{1}\right) \mathcal{D}_{G^{\prime}}\left(S_{2}\right)}{\mathcal{D}_{G^{\prime}}\left(S_{3} \mid S_{4}\right)}$ is constructed by $\frac{\Rightarrow t \quad \frac{A \Rightarrow A}{A, t \Rightarrow A}\left(t_{l}\right)}{A \Rightarrow A}($ CUT $)$.

$$
\text { Let } \frac{G^{\prime} G^{\prime \prime}}{G^{\prime \prime \prime}}(C O M) \text { be } \frac{B \Rightarrow B\left(\begin{array}{c}
\Rightarrow p_{2}, p_{4}, \neg A \odot \neg A\left|p_{1}, p_{2} \Rightarrow A \odot A\right| \\
A \Rightarrow p_{1}\left|A \Rightarrow p_{3}\right| p_{3}, p_{4} \Rightarrow A \odot A
\end{array}\right)}{\left(\begin{array}{c}
\Rightarrow p_{2}, B\left|B \Rightarrow p_{4}, \neg A \odot \neg A\right| A \Rightarrow p_{1} \mid \\
p_{1}, p_{2} \Rightarrow A \odot A\left|A \Rightarrow p_{3}\right| p_{3}, p_{4} \Rightarrow A \odot A
\end{array}\right)}(C O M) \text {, }
$$

where $G^{\prime}=S_{1}=B \Rightarrow B ; G_{2}=p_{1}, p_{2} \Rightarrow A \odot A\left|A \Rightarrow p_{1}\right| A \Rightarrow p_{3} \mid p_{3}, p_{4} \Rightarrow A \odot A$; $S_{2}=\Rightarrow p_{2}, p_{4}, \neg A \odot \neg A ; G^{\prime \prime}=G_{2} \mid S_{2} ; S_{3}=\Rightarrow p_{2}, B ; S_{4}=B \Rightarrow p_{4}, \neg A \odot \neg A$ and $G^{\prime \prime \prime}=G_{2}\left|S_{3}\right| S_{4}$. Then $\mathcal{D}_{G^{\prime}}\left(S_{1}\right)=B \Rightarrow B ; \mathcal{D}_{G^{\prime \prime}}\left(S_{2}\right)=A, A \Rightarrow A \odot A, \neg A \odot \neg A, A \odot A ; \mathcal{D}_{G^{\prime \prime \prime}}\left(S_{3}\right)=A \Rightarrow B, A \odot A ; \mathcal{D}_{G^{\prime \prime \prime}}\left(S_{4}\right)=$ $A, B \Rightarrow A \odot A, \neg A \odot \neg A ; \mathcal{D}_{G^{\prime \prime \prime}}\left(S_{3} \mid S_{4}\right)=\mathcal{D}_{G^{\prime \prime \prime}}\left(S_{4}\right) \mid \mathcal{D}_{G^{\prime \prime \prime}}\left(S_{4}\right)$. 
Thus the proof of $\frac{\mathcal{D}_{G^{\prime}}\left(S_{1}\right) \mathcal{D}_{G^{\prime}}\left(S_{2}\right)}{\mathcal{D}_{G^{\prime}}\left(S_{3} \mid S_{4}\right)}$ is constructed by

$$
\frac{B \Rightarrow B \quad A, A \Rightarrow A \odot A, \neg A \odot \neg A, A \odot A}{A \Rightarrow B, A \odot A \mid A, B \Rightarrow A \odot A, \neg A \odot \neg A}(C O M) .
$$

Appendix A.5.2. Illustration of Construction 3

Let $\tau^{*}$ be

$$
\begin{aligned}
& \frac{H_{8} \equiv B \Rightarrow B H_{9} \equiv A \Rightarrow A}{H_{4} \equiv A \Rightarrow B \mid B \Rightarrow A}(\mathrm{COM}) \frac{H_{10} \equiv B \Rightarrow B H_{11} \equiv A \Rightarrow A}{H_{5} \equiv A \Rightarrow B \mid B \Rightarrow A}(\mathrm{COM})
\end{aligned}
$$

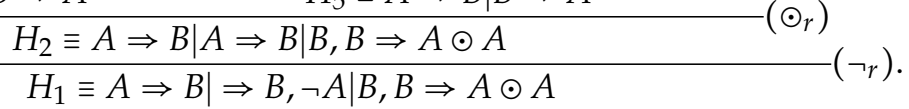

By Construction $3, \tau^{* *}$ is then given as follows.

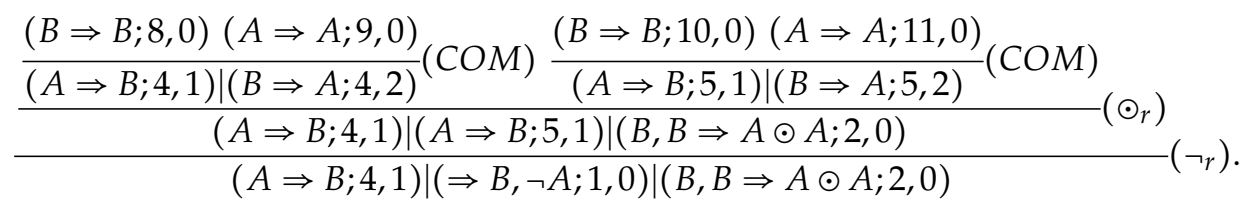

As an example, we calculate $\wp\left(H_{8}\right)$. Since $T h\left(H_{8}\right)=\left(H_{8}, H_{4}, H_{2}, H_{1}\right)$, then $b_{3}=1, b_{2}=b_{1}=b_{0}=0$ by Definition 10 . Thus $\wp\left(H_{8}\right)=b_{0} 2^{0}+b_{1} 2^{1}+b_{2} 2^{2}+b_{3} 2^{3}=8$.

Note that we cannot distinguish the one from the other for two $A \Rightarrow B^{\prime} s$ in $H_{2} \in \tau^{*}$. If we divide $H_{2}$ into $H^{\prime} \mid H^{\prime \prime}$, where $H^{\prime} \equiv A \Rightarrow B$ and $H^{\prime \prime} \equiv A \Rightarrow B \mid B, B \Rightarrow A \odot A$, then $H^{\prime} \cap H^{\prime \prime}=\{A \Rightarrow B\}$ in the conventional meaning of hypersequents. Thus only in the sense that we treat $\tau^{*}$ as $\tau^{* *}$, the assertion that $H^{\prime} \cap H^{\prime \prime}=\varnothing$ for any $H^{\prime} \mid H^{\prime \prime} \subseteq H$ in Proposition 3 holds.

Appendix A.5.3. Illustration of Notation 7 and Construction 4

Let $I=\left\{H_{1}^{c}, H_{2}^{c}\right\}, I_{l}=\left\{H_{1}^{c}\right\}, I_{r}=\left\{H_{2}^{c}\right\}, \mathcal{I}=\left\{S_{11}^{c}, S_{21}^{c}\right\}, \mathcal{I}_{l}=\left\{S_{11}^{c}\right\}, \mathcal{I}_{r}=\left\{S_{21}^{c}\right\}$,

$$
\frac{G^{\prime}\left|S^{\prime} G^{\prime \prime}\right| S^{\prime \prime}}{G^{\prime}\left|G^{\prime \prime}\right| H^{\prime}}\left(\odot_{r}\right) \in \tau^{*},
$$

where $G^{\prime}\left|G^{\prime \prime}\right| H^{\prime}=H_{I}^{V} ; G^{\prime} \equiv A \Rightarrow p_{1}\left|p_{1}, p_{2} \Rightarrow A \odot A ; S^{\prime} \equiv \Rightarrow p_{2}, \neg A ; G^{\prime \prime} \equiv A \Rightarrow p_{3}\right| p_{3}, p_{4} \Rightarrow A \odot A ; S^{\prime \prime} \equiv \Rightarrow$ $p_{4}, \neg A ; H^{\prime} \equiv \Rightarrow p_{2}, p_{4}, \neg A \odot \neg A$ (See Figure 4).

$\left\langle G^{\prime} \mid S^{\prime}\right\rangle_{\mathcal{I}_{1}}=\Rightarrow p_{2}, \neg A ;\left\langle G^{\prime}\right\rangle_{\mathcal{I}_{1}}=\varnothing ;\left\langle G^{\prime}\left|G^{\prime \prime}\right| H^{\prime}\right\rangle_{\mathcal{I}_{1}}=A \Rightarrow p_{3}\left|\Rightarrow p_{2}, p_{4}, \neg A \odot \neg A\right| p_{3}, p_{4} \Rightarrow A \odot$ $A ;\left\langle G \mid G^{*}\right\rangle_{\mathcal{I}_{l}}=G_{\mathcal{I}_{l}}^{*}=G_{S_{11}^{c}}^{*} \Rightarrow p_{2}, B\left|B \Rightarrow p_{4}, \neg A \odot \neg A\right| A \Rightarrow p_{3} \mid p_{3}, p_{4} \Rightarrow A \odot A$ (See Figure 5).

$\left\langle G^{\prime \prime} \mid S^{\prime \prime}\right\rangle_{\mathcal{I}_{r}}=\Rightarrow p_{4}, \neg A ;\left\langle G^{\prime}\left|G^{\prime \prime}\right| H^{\prime}\right\rangle_{\mathcal{I}_{r}}=A \Rightarrow p_{1}\left|\Rightarrow p_{2}, p_{4}, \neg A \odot \neg A\right| p_{1}, p_{2} \Rightarrow A \odot A ;$

$\left\langle G \mid G^{*}\right\rangle_{\mathcal{I}_{r}}=G_{\mathcal{I}_{r}}^{*}=G_{S_{21}^{c}}^{*}=A \Rightarrow p_{1}\left|\Rightarrow p_{2}, B\right| B \Rightarrow p_{4}, \neg A \odot \neg A\left|p_{1} \Rightarrow C\right| C, p_{2} \Rightarrow A \odot A$ (See Figure 8).

$\left\langle G^{\prime}\left|G^{\prime \prime}\right| H^{\prime}\right\rangle_{\mathcal{I}}=\Rightarrow p_{2}, p_{4}, \neg A \odot \neg A ;\left\langle G \mid G^{*}\right\rangle_{\mathcal{I}}=G_{\mathcal{I}}^{*}=G_{\left\{s_{11}^{c}, s_{21}^{c}\right\}}^{*}=G_{\mathcal{I}_{l}}^{*} \cap G_{\mathcal{I}_{r}}^{*} \Rightarrow p_{2}, B \mid B \Rightarrow p_{4}, \neg A \odot \neg A$ (See Figure 10).

Appendix A.5.4. Illustration of Theorem 2

Note that sequents in [] are principal sequents of elimination rules in the following. Let $I, I_{r}, I_{l}$ be the same as in Appendix A.5.3 and, $\mathbf{I}=\left\{\left\lceil S_{1}^{c}\right\rceil_{I},\left[S_{2}^{c}\right]_{I}\right\}, \mathbf{I}_{l}=\left\{\left[S_{1}^{c}\right]_{I}\right\}, \mathbf{I}_{r}=\left\{\left\lceil S_{2}^{c}\right]_{I}\right\}$,

$$
\begin{gathered}
\left\lceil S_{1}^{c}\right\rceil_{I}=G_{H_{2}^{c}: G \mid G^{*}}^{\dot{\zeta}}=A \Rightarrow p_{5}\left|\Rightarrow p_{6}, B\right| B \Rightarrow p_{8}, \neg A \odot \neg A\left|p_{5} \Rightarrow C\right| \\
C, p_{6} \Rightarrow A \odot A\left|B \Rightarrow p_{7}, \neg A \odot \neg A\right| p_{7} \Rightarrow C \mid C, p_{8} \Rightarrow A \odot A, \\
\left\lceil S_{2}^{c}\right]_{I}=G_{H_{1}^{c}: G \mid G^{*}}^{\not{c}}=\Rightarrow p_{2}, B\left|B \Rightarrow p_{4}, \neg A \odot \neg A\right| p_{1} \Rightarrow C\left|C, p_{2} \Rightarrow A \odot A\right|
\end{gathered}
$$




$$
\begin{aligned}
& A \Rightarrow p_{3}\left|\Rightarrow p_{1}, B\right| p_{3} \Rightarrow C \mid C, p_{4} \Rightarrow A \odot A \text {. }
\end{aligned}
$$

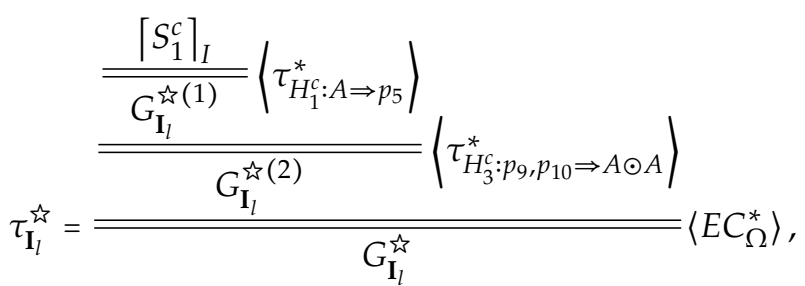

where

$$
\begin{aligned}
& G_{\mathbf{I}_{l}}^{\hbar(1)}=\left[\Rightarrow p_{5}, B\left|B \Rightarrow p_{10}, \neg A \odot \neg A\right| A \Rightarrow p_{9} \mid p_{10}, p_{9} \Rightarrow A \odot A\right]\left|\Rightarrow p_{6}, B\right| \\
& B \Rightarrow p_{8}, \neg A \odot \neg A\left|p_{5} \Rightarrow C\right| C, p_{6} \Rightarrow A \odot A\left|B \Rightarrow p_{7}, \neg A \odot \neg A\right| \\
& p_{7} \Rightarrow C \mid C, p_{8} \Rightarrow A \odot A \text {, } \\
& G_{\mathbf{I}_{l}}^{\varkappa(2)}=\Rightarrow p_{5}, B\left|B \Rightarrow p_{10}, \neg A \odot \neg A\right| A \Rightarrow p_{9}\left|\left[p_{9} \Rightarrow C \mid C, p_{10} \Rightarrow A \odot A\right]\right| \\
& \Rightarrow p_{6}, B\left|B \Rightarrow p_{8}, \neg A \odot \neg A\right| p_{5} \Rightarrow C\left|C, p_{6} \Rightarrow A \odot A\right| \\
& B \Rightarrow p_{7}, \neg A \odot \neg A\left|p_{7} \Rightarrow C\right| C, p_{8} \Rightarrow A \odot A \text {, } \\
& G_{\mathbf{I}_{I_{j}}}^{\tilde{\mu}}=\Rightarrow p_{5}, B\left|A \Rightarrow p_{9}\right| p_{9} \Rightarrow C\left|\Rightarrow p_{6}, B\right| B \Rightarrow p_{8}, \neg A \odot \neg A \mid \\
& p_{5} \Rightarrow C\left|C, p_{6} \Rightarrow A \odot A\right| B \Rightarrow p_{7}, \neg A \odot \neg A\left|p_{7} \Rightarrow C\right| C, p_{8} \Rightarrow A \odot A \text {, } \\
& G_{H^{V}: G^{\prime \prime}}^{\text {峛 }(J)}=A \Rightarrow p_{9}\left|p_{9} \Rightarrow C\right| C, p_{10} \Rightarrow A \odot A ; \widehat{S^{\prime \prime}}=B \Rightarrow p_{10}, \neg A \odot \neg A ; \widehat{S^{\prime}}=\Rightarrow p_{5}, B ; \\
& G_{H_{I}^{V}: H^{\prime}}^{\xi(J)}=G_{H_{I}^{V}: H^{\prime}}^{*}=\widehat{S^{\prime}}\left|\widehat{S^{\prime \prime}} ; G_{\dagger}=A \Rightarrow p_{9}\right| p_{9} \Rightarrow C\left|C, p_{10} \Rightarrow A \odot A\right| B \Rightarrow p_{10}, \neg A \odot \neg A \text {. }
\end{aligned}
$$

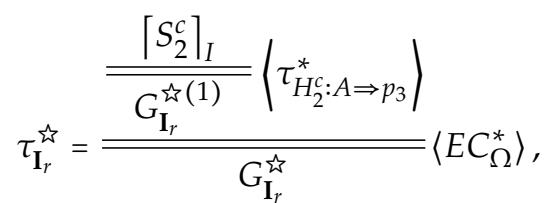

where

$$
\begin{gathered}
G_{\mathbf{I}_{r}}^{\hat{\imath}(1)}=\Rightarrow p_{2}, B\left|B \Rightarrow p_{4}, \neg A \odot \neg A\right| p_{1} \Rightarrow C\left|C, p_{2} \Rightarrow A \odot A\right| \\
\Rightarrow p_{1}, B\left|p_{3} \Rightarrow C\right| C, p_{4} \Rightarrow A \odot A \mid\left[A \Rightarrow p_{11}\left|\Rightarrow p_{12}, B\right|\right. \\
\left.\quad B \Rightarrow p_{3} \neg A \odot \neg A\left|p_{11} \Rightarrow C\right| C, p_{12} \Rightarrow A \odot A\right], \\
G_{\mathbf{I}_{r}}^{\text {虫 }} \Rightarrow p_{2}, B\left|B \Rightarrow p_{4}, \neg A \odot \neg A\right| p_{1} \Rightarrow C\left|C, p_{2} \Rightarrow A \odot A\right| \\
\Rightarrow p_{1}, B\left|p_{3} \Rightarrow C\right| C, p_{4} \Rightarrow A \odot A\left|A \Rightarrow p_{11}\right| B \Rightarrow p_{3}, \neg A \odot \neg A \mid p_{11} \Rightarrow C .
\end{gathered}
$$

Since there is only one elimination rule in $\tau_{\mathbf{I}_{r}}^{\tau^{2}}$, the case we need to process is $\tau_{H_{2}^{c}}^{*}: A \Rightarrow p_{3}$, i.e.,

$$
\tau_{\mathbf{I}_{\mathbf{j} r}}^{*}=\frac{\left\lceil S_{2}^{c}\right\rceil_{I}}{G_{H_{2}^{c}:\left\lceil S_{2}^{c}\right\rceil_{I}}^{\hbar(1)}}\left\langle\tau_{H_{2}^{c}: A \Rightarrow p_{3}}^{*}\right\rangle .
$$

Then $v=1, S_{j_{r 1}}^{c}=A \Rightarrow p_{3} ; G_{b_{r 1}}=\Rightarrow p_{2}, B\left|B \Rightarrow p_{4}, \neg A \odot \neg A\right| p_{1} \Rightarrow C \mid$

$$
\mathrm{C}, p_{2} \Rightarrow A \odot A\left|\Rightarrow p_{1}, B\right| p_{3} \Rightarrow C \mid C, p_{4} \Rightarrow A \odot A \text { in } \tau_{\mathbf{I}_{\mathbf{j} r}}^{*}
$$

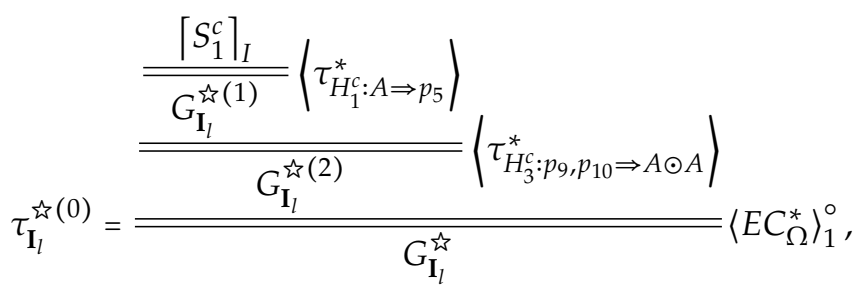




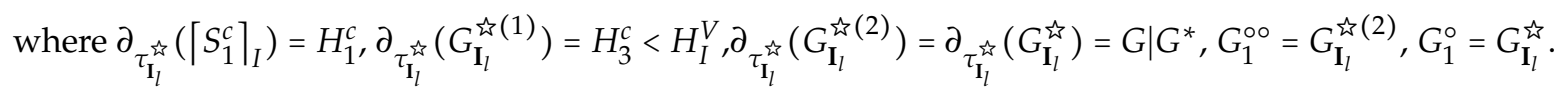

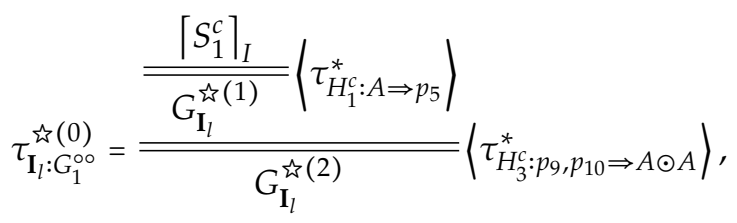

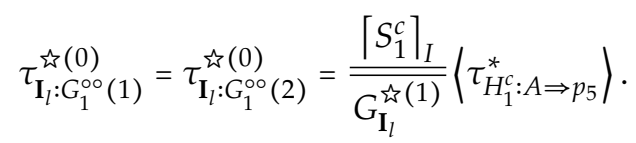

Since there is only one elimination rule in $\tau_{\mathbf{I}_{l}: G_{1}^{\circ o}(2)}^{i(0)}$, the case we need to process is $\tau_{H_{1}^{c}: A \Rightarrow p_{5}}^{*}$,i.e.,

$$
\tau_{\mathbf{I}_{\mathbf{j}_{l}}}^{*}=\frac{\left\lceil S_{1}^{c}\right\rceil_{I}}{\overline{G_{\mathbf{I}_{l}}^{\tilde{j}(1)}}}\left\langle\tau_{H_{1}^{c}: A \Rightarrow p_{5}}^{*}\right\rangle \text {. }
$$

Then $u=1, S_{j_{l 1}}^{c}\left(t_{l 1}\right)=A \Rightarrow p_{5} ; G_{b_{l 1}}=\Rightarrow p_{6}, B\left|B \Rightarrow p_{8}, \neg A \odot \neg A\right|$

$$
p_{5} \Rightarrow C\left|C, p_{6} \Rightarrow A \odot A\right| B \Rightarrow p_{7}, \neg A \odot \neg A\left|p_{7} \Rightarrow C\right| C, p_{8} \Rightarrow A \odot A \text { in } \tau_{\mathbf{I}_{\mathbf{j}_{l}}}^{*} .
$$

$\tau_{\mathbf{I}_{\mathbf{j}_{l}}}^{*}$ is replaced with $\tau_{\mathbf{I}_{\mathbf{j}_{l}}}^{*} \mathbf{I}_{\mathbf{j}_{r}}$ in Step 3 of Stage 1, i.e.,

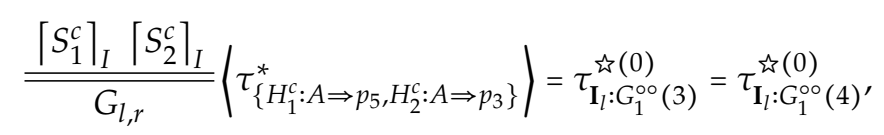

where

$$
\begin{aligned}
G_{l, r} & \Rightarrow p_{5}, B\left|B \Rightarrow p_{3}, \neg A \odot \neg A\right| G_{b_{r 1}} \mid G_{b_{11}}= \\
& \Rightarrow p_{2}, B\left|B \Rightarrow p_{4}, \neg A \odot \neg A\right| p_{1} \Rightarrow C\left|C, p_{2} \Rightarrow A \odot A\right| \Rightarrow p_{1}, B \mid \\
p_{3} & \Rightarrow C\left|C, p_{4} \Rightarrow A \odot A\right| \Rightarrow p_{6}, B\left|B \Rightarrow p_{8}, \neg A \odot \neg A\right| \\
p_{5} & \Rightarrow C\left|C, p_{6} \Rightarrow A \odot A\right| B \Rightarrow p_{7}, \neg A \odot \neg A\left|p_{7} \Rightarrow C\right| \\
& \Rightarrow C, p_{8} \Rightarrow A \odot A\left|p_{5}, B\right| B \Rightarrow p_{3}, \neg A \odot \neg A .
\end{aligned}
$$

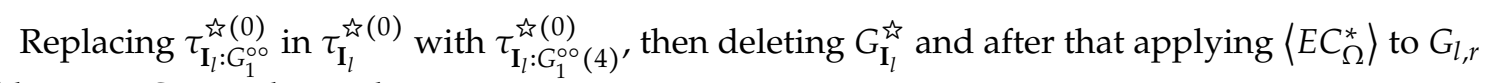
and keeping $G_{b_{r 1}}$ unchanged, we get

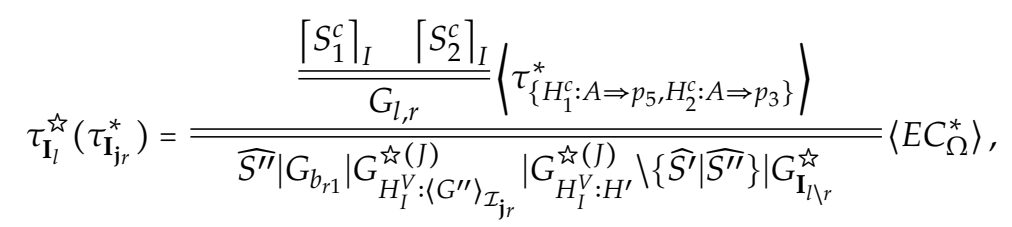

where $G_{H_{I}^{V}:\left\langle G^{\prime \prime}\right\rangle_{\mathcal{I}_{\mathbf{j} r}}}^{\stackrel{\tilde{j}}{(J)}}=G_{H_{I}^{V}:\left\langle G^{\prime \prime}\right\rangle_{\mathcal{I}_{\mathbf{j} r}}}^{*}=\varnothing ; \widehat{S^{\prime}}=\Rightarrow p_{5}, B$;

$$
\begin{aligned}
& \widehat{S^{\prime \prime}}=B \Rightarrow p_{3}, \neg A \odot \neg A ; G_{\ddagger}=G_{b_{r 1}}\left|\widehat{S^{\prime \prime}} ; G_{H_{I}^{V}: H^{\prime}}^{\text {मे }(J)}=G_{H_{I}^{V}: H^{\prime}}^{*}=\widehat{S^{\prime}}\right| \widehat{S^{\prime \prime}} ; \\
& G_{\mathbf{I}_{l \mid r}}^{\hbar_{3}}=\Rightarrow p_{5}, B\left|\Rightarrow p_{6}, B\right| B \Rightarrow p_{7}, \neg A \odot \neg A\left|p_{5} \Rightarrow C\right| C, p_{6} \Rightarrow A \odot A \mid \\
& p_{7} \Rightarrow C\left|C, p_{8} \Rightarrow A \odot A\right| B \Rightarrow p_{8}, \neg A \odot \neg A \text {. }
\end{aligned}
$$

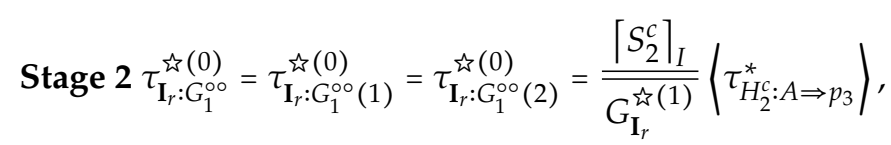




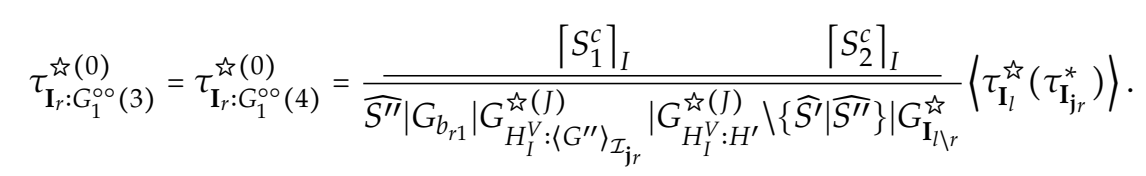

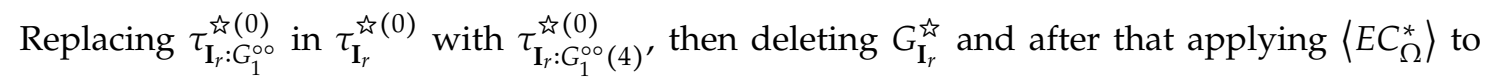

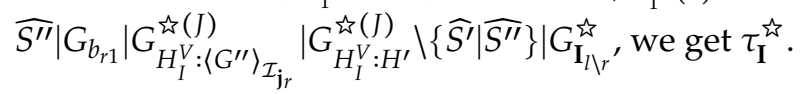

\section{References}

1. Rose, A.; Rosser, J.B. Fragments of many-valued statement calculi. Trans. Am. Math. Soc. 1958, 87, 1-53. [CrossRef]

2. Chang, C.C. Algebraic analysis of many-valued logics. Trans. Am. Math. Soc. 1958, 88, 467-490. [CrossRef]

3. Chang, C.C. A new proof of the completeness of the Lukasiewicz's axioms. Trans. Am. Math. Soc. 1959, 93, 74-80. [CrossRef]

4. Hájek, P. Metamathematics of Fuzzy Logic; Kluwer: Dordrecht, The Netherlands, 1998.

5. Hájek, P. Basic fuzzy logic and BL-algebras. Soft Comput. 1998, 2, 124-128. [CrossRef]

6. Cignoli, R.; Esteva, F.; Godo, L.; Torrens, A. Basic fuzzy logic is the logic of continuous t-norms and their residua. Soft Comput. 2000, 4, 106-112. [CrossRef]

7. Esteva, F.; Godo, L. Monoidal t-norm based logic: towards a logic for left-continuous t-norms. Fuzzy Sets Syst. 2001, 124, 271-288. [CrossRef]

8. Jenei, S.; Montagna, F. A proof of standard completeness for Esteva and Godo's logic MTL. Stud. Log. 2002, 70, 183-192. [CrossRef]

9. Metcalfe, G.; Montagna, F. Substructural fuzzy logics. J. Symb. Log. 2007, 7, 834-864. [CrossRef]

10. Cintula, P.; Noguera, C. Implicational (semilinear) logics I: A new hierarchy. Arch. Math. Log. 2010, 49, 417-446. [CrossRef]

11. Takeuti, G.; Titani, T. Intuitionistic fuzzy logic and intuitionistic fuzzy set theory. J. Symb. Log. 1984, 49, 851-866. [CrossRef]

12. Avron, A. A constructive analysis of RM. J. Symb. Log. 1987, 52, 939-951. [CrossRef]

13. Pottinger, G. Uniform cut-free formulations of T, S4 and S5 (abstract). J. Symb. Log. 1983, 48, 900-901.

14. Avron, A. Hypersequents, logical consequence and intermediate logics for concurrency. Ann. Math. Artif. Intell. 1991, 4, 225-248. [CrossRef]

15. Esteva, F.; Gispert, J.; Godo, L.; Montagna, F. On the standard and rational completeness of some axiomatic extensions of the monoidal t-norm logic. Stud. Log. 2002, 71, 199-226.:1016548805869. [CrossRef]

16. Wang, S.M. Involutive uninorm logic with the $n$-potency axiom. Fuzzy Sets Syst. 2013, 218, 1-23. [CrossRef]

17. Wang, S.M. The Finite Model Property for Semilinear Substructural Logics. Math. Log. Q. 2013, 59, 268-273. [CrossRef]

18. Jenei, S. Co-rotation constructions of residuated semigroups. Fuzzy Sets Syst. 2014, 252, 25-34. [CrossRef]

19. Ciabattoni, A.; Metcalfe, G. Density elimination. Theor. Comput. Sci. 2008, 403, 328-346. [CrossRef]

20. Wang, S.M. The logic of pseudo-uninorms and their residua. Symmetry 2019, 11, 368. [CrossRef]

21. Metcalfe, G.; Olivetti, N.; Gabbay, D. Proof Theory for Fuzzy Logics; Springer Series in Applied Logic; Springer: New York, NY, USA, 2009; Volume 36, ISBN 9781402094095.

22. Metcalfe, G.; Tsinakis, C. Density revisited. Soft Comput. 2017, 21, 175-189. [CrossRef]

23. Baldi, P.; Terui, K. Densification of FL chains via residuated frames. Algebra Universalis 2016, 75, 169-195. [CrossRef] 